Supporting Information for

\title{
Modeling Mechanisms of Unusual Benzene Imine N6 Adduct Formation in Carcinogenic Reactions of Arylnitrenium Ions with Adenosine
}

\author{
Shi-Fei Qi and Zhong-Zhi Yang* \\ Chemistry and Chemical Engineering Faculty, Liaoning Normal University, Dalian 116029, P.R.China \\ E-mail address: zzyang@ @nnu.edu.cn
}




\section{Table of Contents}

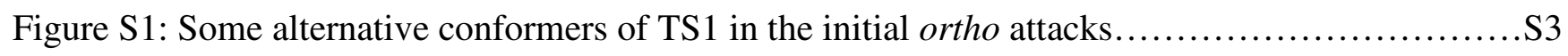

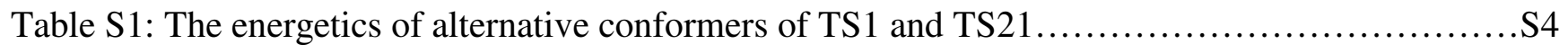

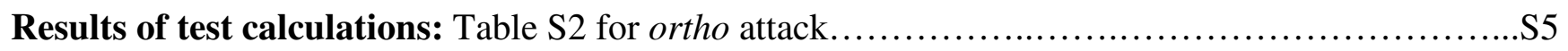

Table S3 for para attack...................................... 6

The discussions of ortho attack to imine and azepine adducts:

Figure S2: The schematic aqueous Gibbs free energy surface of imine adduct formed from ortho

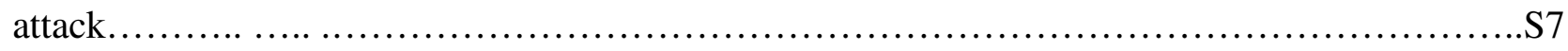

Figure S3: The structures of stationary points related to the formation of imine adduct from ortho

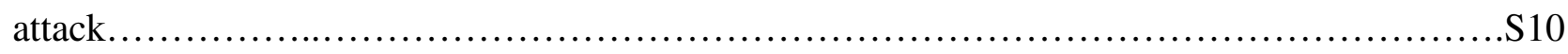

Table S4: The key geometrical parameters of INT and TS optimized using various methods..........S11 

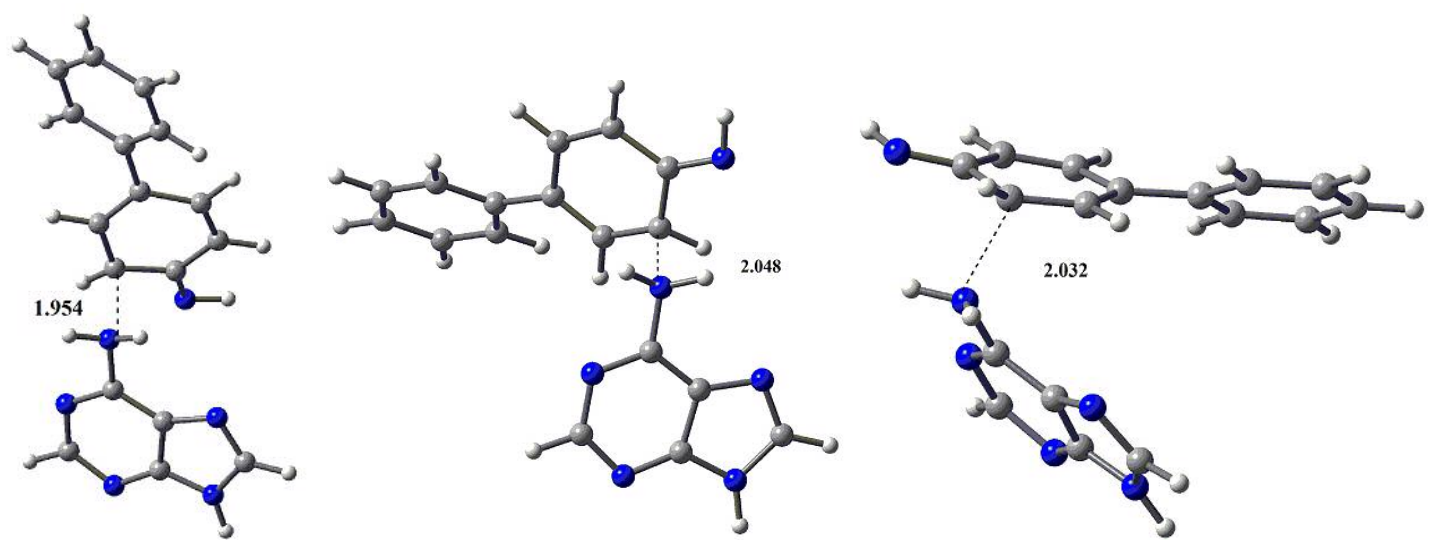

TS1

S-TS1

S2-TS1

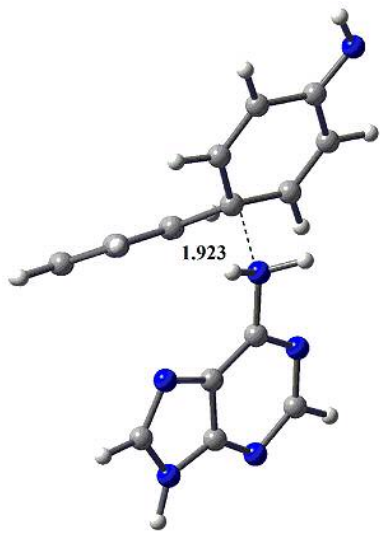

TS21

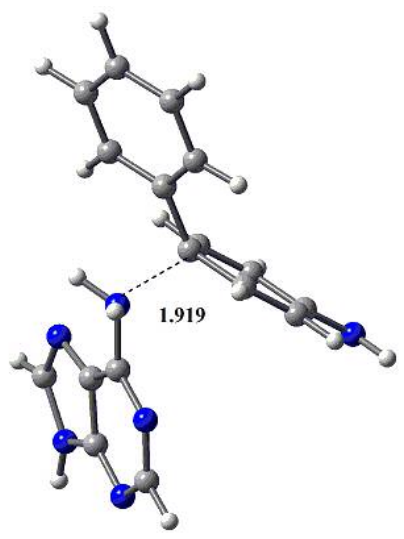

S-TS21

Figure S1. The B3PW91/6-31+G(d,p) optimized geometries of TS1, TS21 and their alternative conformers in the initial attacks of adenine at ortho and para $\mathrm{C}$ sites of 4-biphenylylnitrenium ion 
Table S1. The relative energies and (Gibbs free energies) of some alternative conformers of TS1 and TS21 relative to the separated reactants in the ortho and para attacks evaluated using various DFT and ab initio methods in the reaction of adenine with 4-biphenylylnitrenium ion.

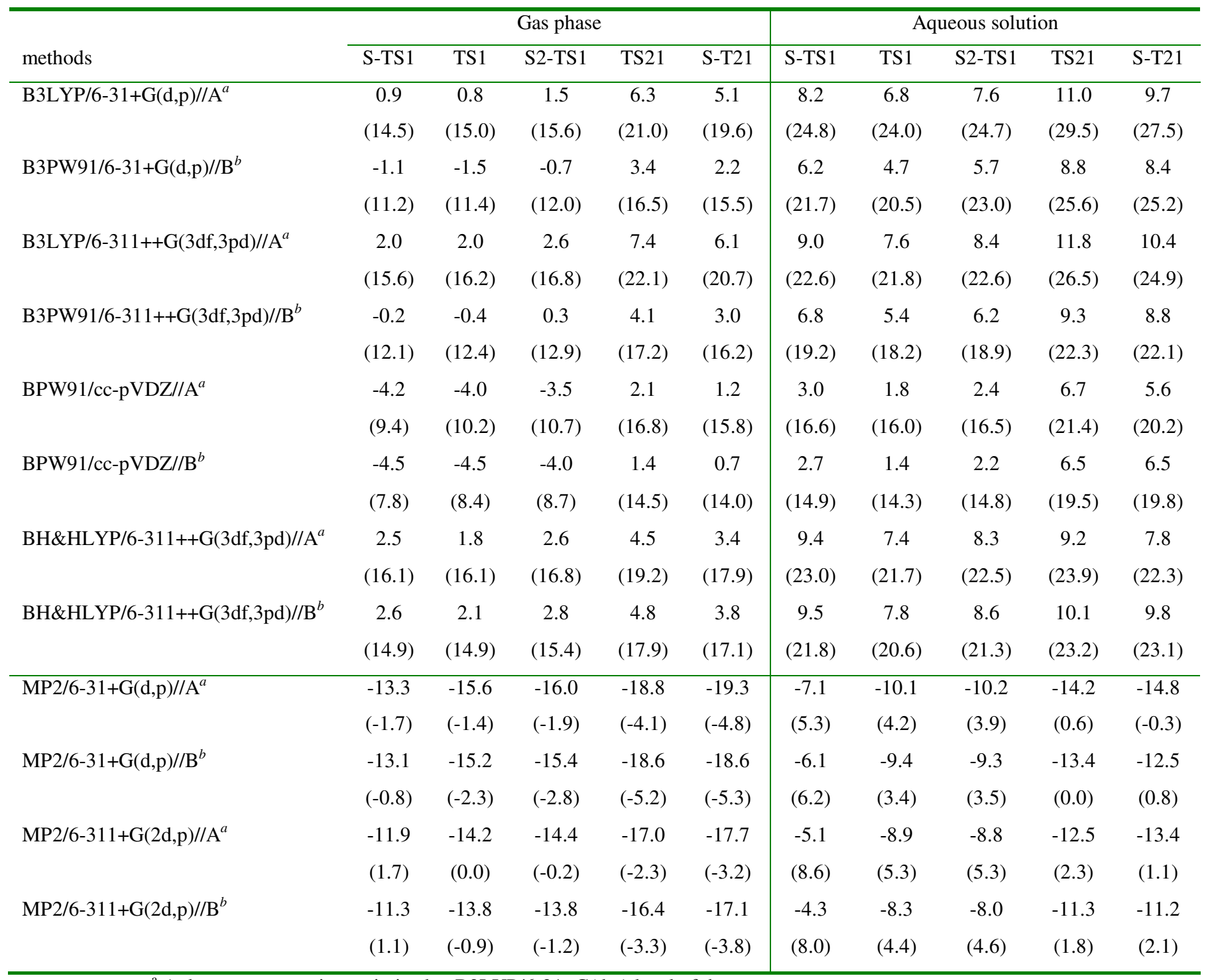

\footnotetext{
${ }^{\mathrm{a}}$ A denotes geometries optimized at B3LYP/6-31+G(d,p) level of theory

${ }^{b}$ B denotes geometries optimized at B3PW91/6-31+G(d,p) level of theory
} 


\section{The results of test calculations for ortho attack and para attack}

Table S2. The gas energies and (Gibbs free energies) of transition states (TS) and intermediates (INT) relative to those of the separated reactants involved in the ortho attack evaluated at various DFT computational levels

\begin{tabular}{|c|c|c|c|c|c|c|c|c|c|c|c|c|c|c|c|}
\hline gas & Com1 & S-Ts1 & S-INT1 & $\mathrm{Ts} 2$ & INT2 & Ts3 & INT3 & Ts4 & INT4 & Ts5 & INT5 & Ts6 & INT6 & Ts7 & INT7 \\
\hline \multirow[t]{2}{*}{ M1 } & -5.5 & -2.3 & -11.5 & -2.9 & -30.0 & -26.3 & -48.3 & -12.3 & -39.0 & -21.0 & -29.0 & 2.9 & -11.9 & 2.9 & -5.0 \\
\hline & $(5.8)$ & $(9.2)$ & $(0.9)$ & $(0.0)$ & $(-16.0)$ & $(-12.9)$ & $(-35.6)$ & $(1.4)$ & $(-26.1)$ & $(-8.0)$ & $(-16.3)$ & (10.1) & $(0.9)$ & (10.6) & (8.1) \\
\hline \multirow[t]{2}{*}{ M2 } & & 0.9 & -7.6 & -8.7 & -26.5 & -22.5 & -45.1 & -6.5 & -35.9 & -17.7 & -25.4 & 0.9 & -8.5 & 0.6 & -1.6 \\
\hline & & (14.5) & $(6.7)$ & $(6.1)$ & $(-12.0)$ & $(-7.1)$ & $(-30.5)$ & $(9.0)$ & $(-21.1)$ & $(-2.3)$ & $(-10.8)$ & (15.7) & $(6.1)$ & (16.0) & (13.4) \\
\hline \multirow[t]{2}{*}{ M3 } & & 1.1 & -7.6 & -8.3 & -26.2 & -22.4 & -45.4 & -6.1 & -36.4 & -18.0 & -25.5 & 1.2 & -8.5 & 0.9 & -1.3 \\
\hline & & (14.7) & $(6.8)$ & $(6.5)$ & $(-11.8)$ & $(-7.0)$ & $(-30.7)$ & $(9.4)$ & $(-21.6)$ & $(-2.6)$ & $(-10.8)$ & (16.1) & (6.1) & (16.3) & (13.6) \\
\hline \multirow[t]{2}{*}{ M4 } & -5.3 & 2.0 & -6.4 & -7.1 & -25.4 & -22.0 & -45.8 & -6.0 & -37.0 & -17.9 & -25.1 & 1.0 & -8.3 & 0.8 & -1.9 \\
\hline & (5.9) & (15.6) & $(7.9)$ & $(7.7)$ & $(-11.0)$ & $(-6.6)$ & $(-31.2)$ & $(9.8)$ & $(-22.3)$ & $(-2.5)$ & $(-10.4)$ & (15.9) & $(6.3)$ & (16.2) & (13.1) \\
\hline \multirow[t]{2}{*}{ M5 } & & -3.5 & -13.9 & -15.4 & -31.5 & -28.6 & -49.9 & -15.4 & -40.8 & -24.0 & -30.6 & -4.3 & -13.5 & -4.6 & -6.4 \\
\hline & & $(8.2)$ & $(-1.1)$ & $(-2.3)$ & $(-18.7)$ & $(-15.0)$ & $(-37.0)$ & $(-1.5)$ & $(-27.8)$ & $(-10.3)$ & $(-17.6)$ & $(9.0)$ & $(-0.4)$ & $(9.2)$ & (6.8) \\
\hline \multirow[t]{2}{*}{ M6 } & & -4.3 & -18.6 & -19.7 & -36.0 & -34.8 & -55.1 & -21.8 & -46.0 & -30.5 & -35.1 & -9.2 & -18.6 & -10.9 & -13.3 \\
\hline & & (7.3) & $(-5.7)$ & $(-6.5)$ & $(-23.2)$ & $(-21.1)$ & $(-42.0)$ & $(-7.9)$ & $(-32.8)$ & $(-16.8)$ & $(-22.1)$ & $(4.0)$ & $(-5.3)$ & (2.9) & $(0.0)$ \\
\hline \multirow[t]{2}{*}{ M7 } & -5.3 & -1.1 & -13.0 & -14.6 & -32.1 & -29.9 & -51.5 & -14.5 & -42.3 & -25.5 & -30.9 & -5.3 & -14.2 & -6.7 & -9.6 \\
\hline & $(5.2)$ & (11.2) & $(0.1)$ & $(-1.0)$ & $(-18.9)$ & $(-15.8)$ & $(-38.2)$ & $(-0.2)$ & $(-28.9)$ & $(-11.3)$ & $(-17.6)$ & $(8.3)$ & $(-0.8)$ & $(7.5)$ & (4.1) \\
\hline \multirow[t]{2}{*}{ M8 } & -5.2 & -0.2 & -12.0 & -13.4 & -31.2 & -29.8 & -52.3 & -14.5 & -43.7 & -26.2 & -30.8 & -5.5 & -14.4 & -6.9 & -10.3 \\
\hline & (5.3) & (12.1) & (1.1) & $(0.2)$ & $(-18.0)$ & $(-15.7)$ & $(-39.0)$ & $(-0.3)$ & $(-30.3)$ & $(-12.0)$ & $(-17.5)$ & $(8.1)$ & $(-1.0)$ & (7.3) & $(3.4)$ \\
\hline
\end{tabular}

M1 denotes B3LYP/6-31G(d,p)// B3LYP/6-31G(d,p) method

M2 denotes B3LYP/6-31+G(d,p)// B3LYP/6-31+G(d,p) method

M3 denotes B3LYP/6-311++G(d,p)// B3LYP/6-31+G(d,p) method

M4 denotes B3LYP/6-311++G(3df,3pd)// B3LYP/6-31+G(d,p) method

M5 denotes B3LYP/cc-pVDZ// B3LYP/cc-pVDZ method

M6 denotes B3PW91/cc-pVDZ // B3PW91/cc-pVDZ method

M7 denotes B3PW91/6-31+G(d,p)// B3PW91/6-31+G(d,p) method

M8 denotes B3PW91/6-311++G(3df,3pd)// B3PW91/6-31+G(d,p) method 
Table S3. The gas energies and (Gibbs free energies) of transition states (TS) and intermediates (INT) relative to those of the separated reactants involved in the para attack evaluated at various DFT computational levels

\begin{tabular}{|c|c|c|c|c|}
\hline gas & Com21 & Ts21 & INT21 & INT22 \\
\hline \multirow[t]{2}{*}{ B3LYP/6-31G(d,p)//A ${ }^{a}$} & & 3.02 & 3.41 & -6.06 \\
\hline & & $(15.81)$ & $(15.91)$ & $(6.85)$ \\
\hline \multirow[t]{2}{*}{ B3LYP/6-31+G(d,p)///B ${ }^{b}$} & -4.4 & 6.31 & 6.71 & -2.70 \\
\hline & $(8.5)$ & $(21.02)$ & $(21.19)$ & (12.18) \\
\hline \multirow[t]{2}{*}{ B3LYP/6-311++G(d,p)//B ${ }^{b}$} & & 6.10 & 6.49 & -2.11 \\
\hline & & (20.81) & (20.98) & (12.77) \\
\hline \multirow[t]{2}{*}{ B3LYP/6-311++G(3df,3pd)/// ${ }^{b}$} & -4.2 & 7.37 & 7.77 & -1.63 \\
\hline & (8.7) & (22.08) & $(22.25)$ & (13.26) \\
\hline \multirow[t]{2}{*}{ B3LYP/cc-pVDZ//C ${ }^{c}$} & & 1.20 & 1.48 & -7.02 \\
\hline & & (14.26) & (14.30) & $(6.15)$ \\
\hline \multirow[t]{2}{*}{ B3PW91/cc-pVDZ /// ${ }^{d}$} & & -0.54 & -1.15 & -13.34 \\
\hline & & $(12.13)$ & (11.64) & $(-0.09)$ \\
\hline \multirow[t]{2}{*}{ B3PW91/6-31+G(d,p)//E } & -4.2 & 3.37 & 3.01 & -10.14 \\
\hline & (7.2) & $(16.45)$ & $(16.22)$ & $(3.52)$ \\
\hline \multirow[t]{2}{*}{ B3PW91/6-311++G(3df,3pd)//E } & & 4.14 & 3.70 & -9.48 \\
\hline & & $(17.21)$ & (16.91) & (4.18) \\
\hline \multicolumn{5}{|c|}{${ }^{a} \mathrm{~A}$ denotes geometries optimized at B3LYP/6-31G(d,p) level of theory } \\
\hline \multicolumn{5}{|c|}{${ }^{b} \mathrm{~B}$ denotes geometries optimized at $\mathrm{B} 3 \mathrm{LYP} / 6-31+\mathrm{G}(\mathrm{d}, \mathrm{p})$ level of theory } \\
\hline \multicolumn{5}{|c|}{${ }^{c} \mathrm{C}$ denotes geometries optimized at B3LYP/cc-pVDZ level of theory } \\
\hline \multicolumn{5}{|c|}{${ }^{d}$ D denotes geometries optimized at B3PW91/cc-pVDZ level of theory } \\
\hline
\end{tabular}




\section{The discussions of ortho attack to imine and azepine adducts}

The schematic aqueous Gibbs free energy surface from an initial attack (route OA) of adenine at ortho C (C14 in Scheme 1) site of 4-biphenylylnitrenium ion is plotted in Figure S3. This attack firstly produces an ion-molecule complex COM1, which proceeds a transition state S-TS1 to form an intermediate S-INT1 that is $9.3 \mathrm{kcal} / \mathrm{mol}$ higher than the separated reactants. As presented in Figure S3, the N6-C14 distances of TS1 and INT1 are respectively $2.048 \AA$ and $1.510 \AA$. Additionally, in the structure of S-INT1, a hydrogen bond is formed between the hydrogen on the N6 of the adenine and the nitrogen (N13) of4-biphenylylnitrenium ion. Hence, the hydrogen transfer from N6 to N13 may be easier. Indeed, the aqueous Gibbs free energy (TS2 relative to S-INT1) for this hydrogen transfer process is only $2.7 \mathrm{kcal} / \mathrm{mol}$. After this step, a tetrahedral intermediate INT2 is formed, and the N6 -C14 bond is formed $(1.500 \AA)$. The tetrahedral intermediate is $-10.5 \mathrm{kcal} / \mathrm{mol}$ more stable than the separated reactants. Following the intermediate INT2, the third transition state TS3 appears. The aqueous Gibbs free energy of TS3 is small at $-5.2 \mathrm{kcal} / \mathrm{mol}$ (relative to the separated reactants). Once the third transition state is past, the energy steeply falls to form the intermediate INT3. It is $-29.4 \mathrm{kcal} / \mathrm{mol}$ more stable than the separated reactants and its energy is the lowest among all species located in this study.

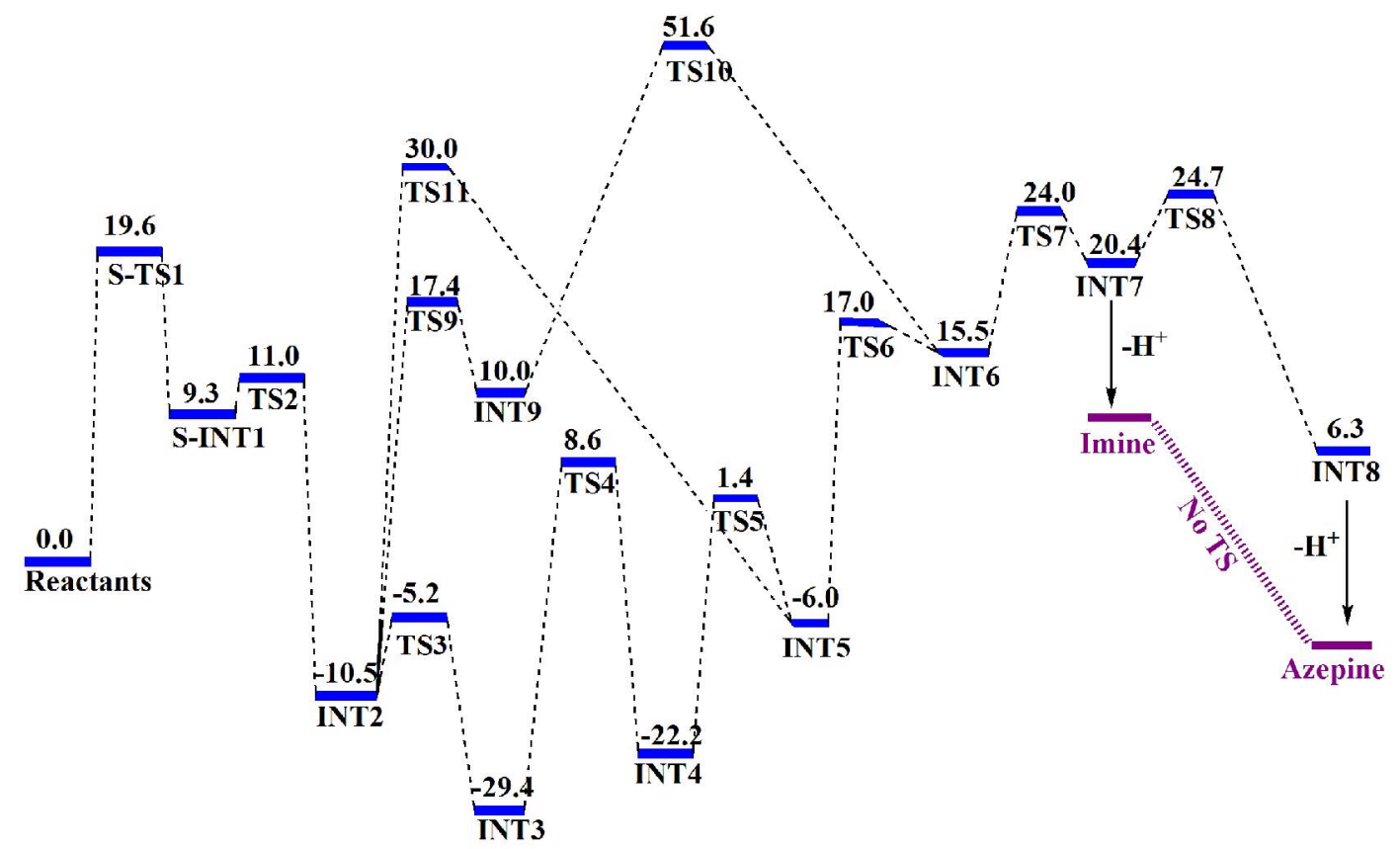

Figure S2. The schematic aqueous Gibbs free energy surface of N6 imine adduct formed by the initial attack of adenine at ortho $\mathrm{C}$ site of 4-biphenylylnitrenium ion at B3LYP/6-31G(d,p) level of theory 
As is shown in Figure S2, following the intermediate INT2, three possible pathways to N1 protonated imine adduct INT7 and azepine adduct INT8 are obtained, i.e. Path 1: INT2 $\rightarrow$ INT3 $\rightarrow$ $\mathrm{INT} 4 \rightarrow \mathrm{INT} 5 \rightarrow$ INT6 $\rightarrow$ INT7 $\rightarrow$ INT8, Path $2:$ INT2 $\rightarrow$ INT9 $\rightarrow$ INT10 $\rightarrow$ INT6 $\rightarrow$ INT7 $\rightarrow$ INT8, and Path 3: INT2 $\rightarrow$ INT5 $\rightarrow$ INT6 $\rightarrow$ INT7 $\rightarrow$ INT8. In the following discussion, we mainly focus on these three reaction pathways.

In the Path 1, the aqueous Gibbs free energy of the third transition state TS3 is small at $-5.2 \mathrm{kcal} / \mathrm{mol}$ (relative to the separated reactants). Once past the third transition state, the energy steeply falls to form the intermediate INT3. It is $-29.4 \mathrm{kcal} / \mathrm{mol}$ more stable than the separated reactants and its energy is the lowest among all species located in this study. After this step, the hydrogen of C14 transfers to N7 atom of adenine. In the next step, another hydrogen-transfer process ( $\mathrm{H}$ of $\mathrm{N} 6$ to $\mathrm{N} 1$ of adenine) happens and leads to the formation of intermediate INT4. Compared with the foregoing hydrogen-transfer process (TS3), this transfer (TS4) needs higher aqueous Gibbs free energy, about $8.6 \mathrm{kcal} / \mathrm{mol}$ relative to the separated reactants. In the next process, a converse hydrogen-transfer process (TS5) with TS3 happens. As is shown in Figure S3, the distances of H-N7 and H-C14 in TS5 are 1.371 and $1.412 \AA$, respectively, whereas the two distances in TS3 are respectively 1.390 and $1.374 \AA$. Obviously, those are two inverse hydrogen-transfer processes. Relative to the separated reactants, the aqueous Gibbs free energy of TS5 is $1.4 \mathrm{kcal} / \mathrm{mol}$, but it is higher than INT4 by $23.6 \mathrm{kcal} / \mathrm{mol}$. After the intermediate INT5 is formed, it undergoes a 1,2-migration (TS6) of adenine group from $\mathrm{C} 14$ atom to $\mathrm{C} 15$ atom of 4biphenylylnitrenium ion forming intermediate INT6, which is actually arylnitrenium-adenine C15 adduct. This is an energy-consumed process, and substantial Gibbs free energy of $23.0 \mathrm{kcal} / \mathrm{mol}$ relative to INT5 for the 1,2-migration step is needed. Through the process of N6-C10 bond formation (TS7), the intermediate INT7 is obtained, i.e. N1 protonated imine adduct. The aqueous Gibbs free energy of TS7 is about $24.0 \mathrm{kcal} / \mathrm{mol}$ relative to the separated reactants. Then, the $\mathrm{N} 1$ protonated imine adduct INT7 either undergoes a N1 deprotonation process forming imine adduct or continuously and easily isomerizes to form N1 protonated azepine adduct, which can also become azepine adduct by a N1 deprotonation process. Thus, a complete pathway to imine or azepine adduct is found. Interestingly, in this study, the TS connecting the conversion between imine adduct and azepine adduct can not be located despite numerous attempts. This conversion can also not be found between imine and azepine adducts in the reaction of N9-methanol substituted adenine with 4-biphenylylnitrenium ion. 


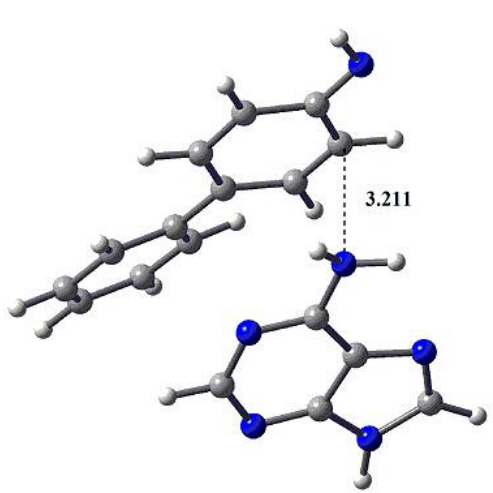

COM1

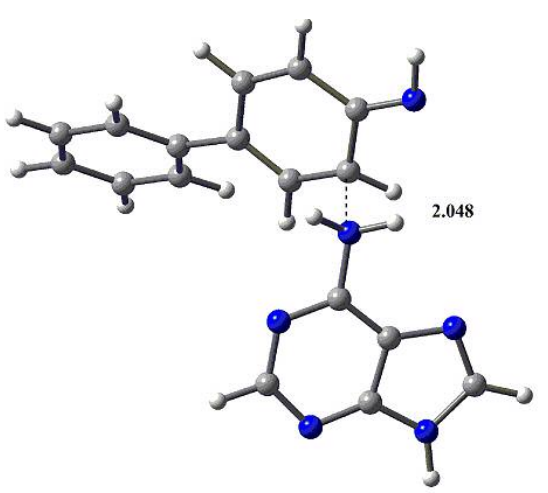

S1-TS1

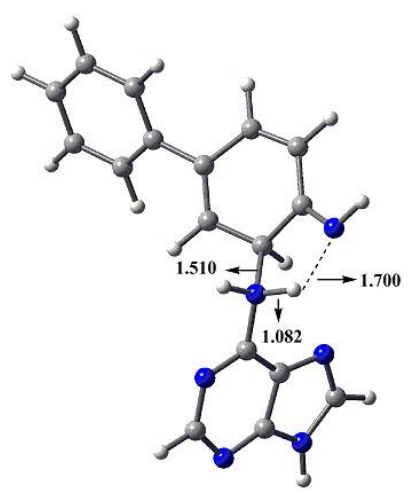

S1-INT1

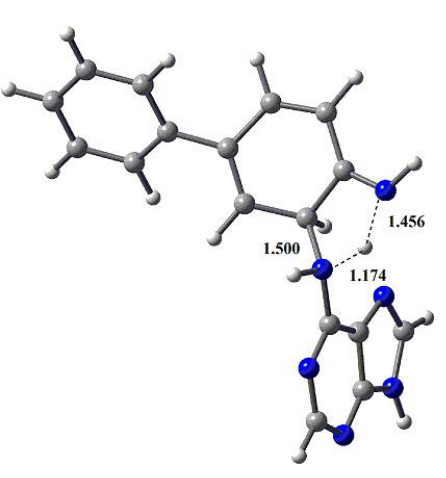

TS2

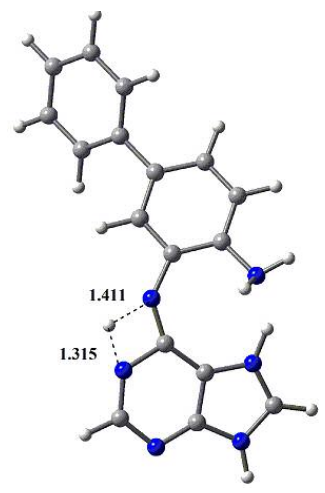

TS4

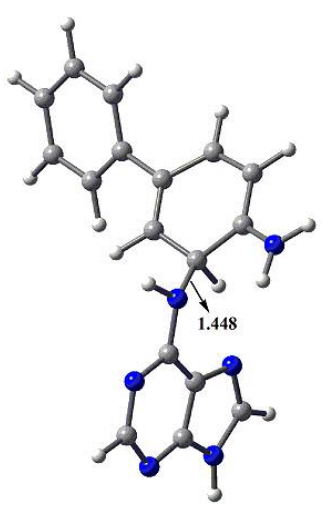

INT2

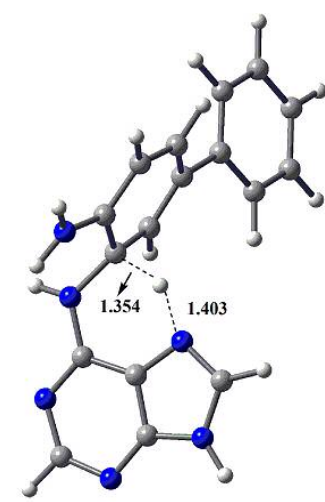

TS3

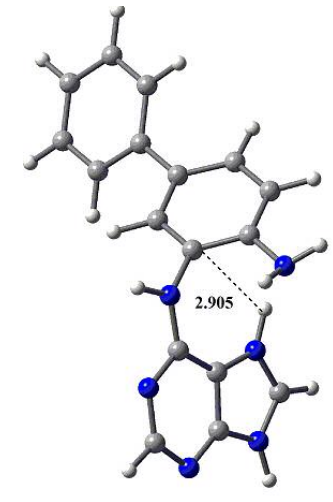

INT3

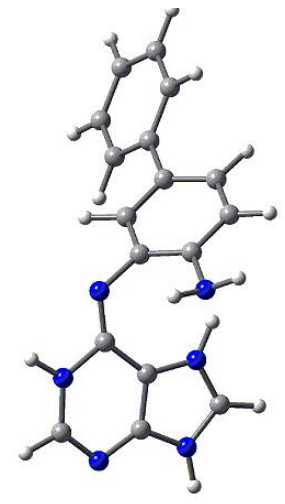

INT4

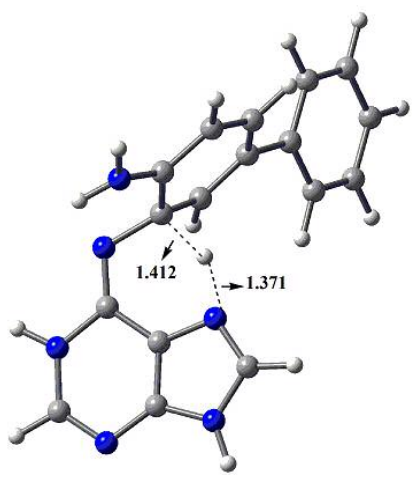

TS5

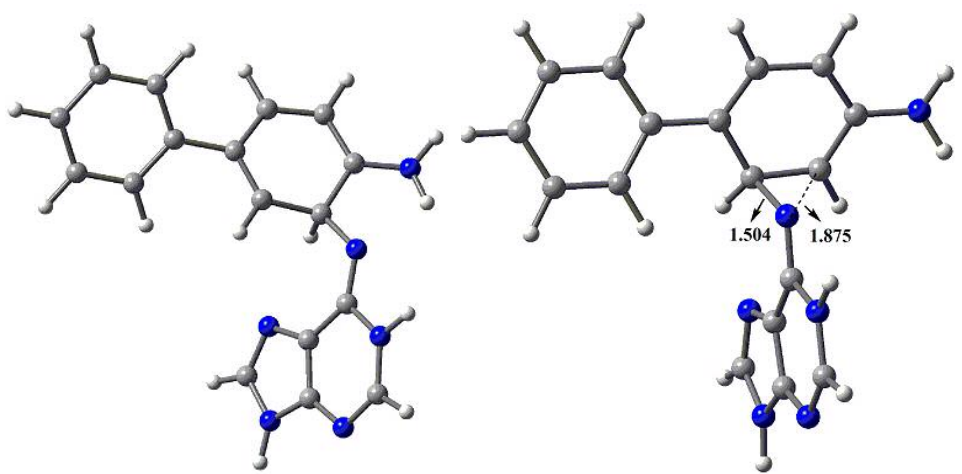

INT5

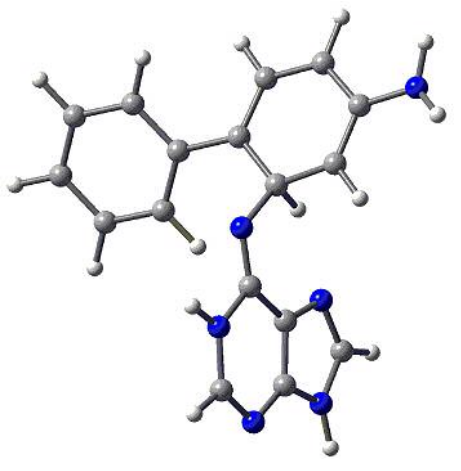

INT6 


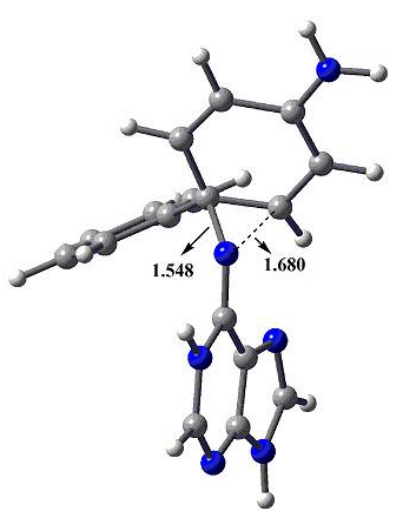

TS7

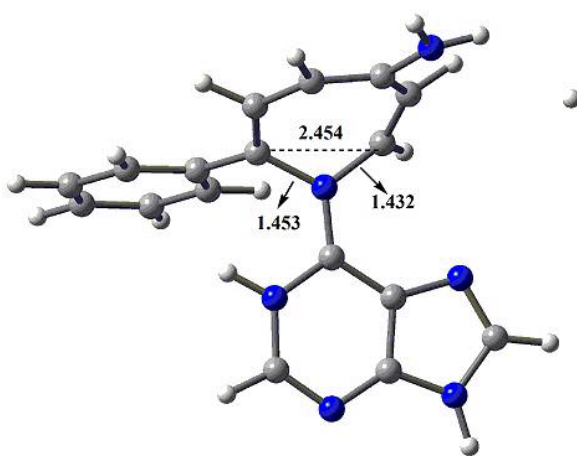

INT8

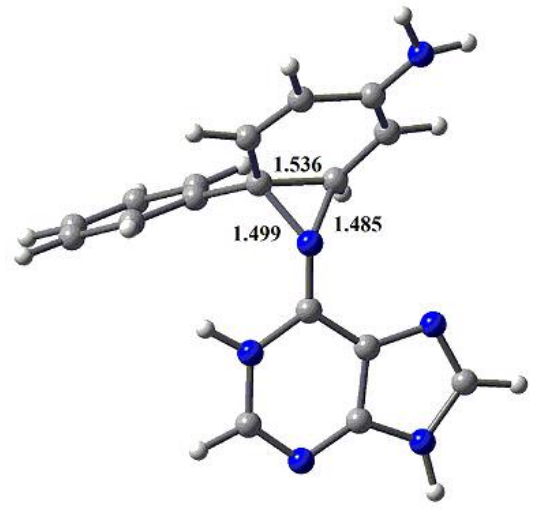

INT7

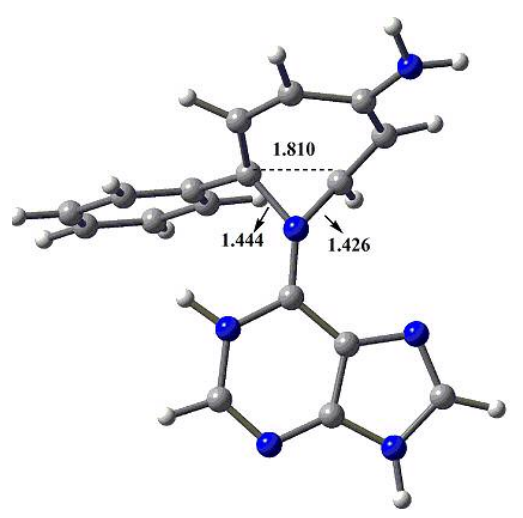

TS8

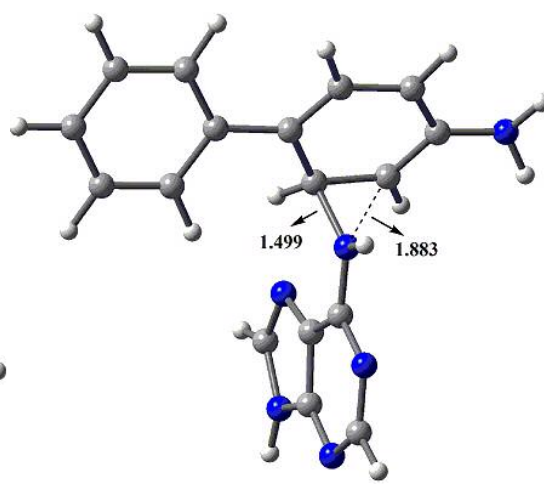

TS9

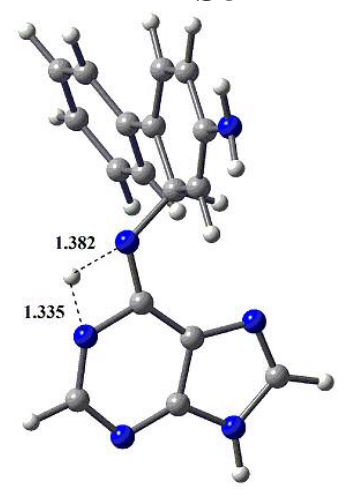

TS10

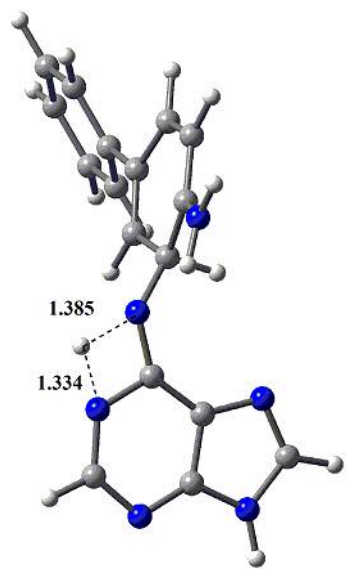

TS11

Figure S3. The B3LYP/6-31+G(d,p) optimized geometries of transition states (TS), and intermediate (INT) in the initial attack (Route $\mathrm{OA}$ ) of adenine at ortho $\mathrm{C}$ site of 4-biphenylylnitrenium ion

Alternatively, intermediate INT2 can directly isomerize to the intermediate INT9 via the 1,2migration of adenine group (TS9). Then the INT9 undergoes a H-transfer step from N6 to N1 of adenine (TS10) to form intermediate INT6. As can be seen from Figure S1, the following steps are as the same as the Path 1, i.e. from INT6 to INT7, then to INT8. The above steps constitute an alternative Path 2 to imine or azepine adduct. In this pathway, H transfer from N6 to N1 of adenine via TS10 is 
energetically unfavorable due to higher Gibbs free energy of $51.6 \mathrm{kcal} / \mathrm{mol}$ relative to the separated reactants.

The third pathway to the imine or azepine adduct is the Path 3. The intermediate INT2 can firstly undergo a H-transfer process from N6 to N1 of adenine (TS11) to directly form intermediate INT5. The subsequent steps of the imine or azepine adduct formation are the same as those in the Path 1. Compared with the Path 1, the aqueous Gibbs free energy of TS11 is higher, about $30.0 \mathrm{kcal} / \mathrm{mol}$ relative to the separated reactants, than those of all TSs involved in the Path 1. Hence, the Path3 seems energetically inaccessible due to this higher barrier.

Table S4 The key geometrical parameters of INT22 and TS21 optimized using various methods. Numbering scheme can be found in Scheme 1.

\begin{tabular}{lcccccc}
\hline & \multicolumn{3}{c}{ INT22 } & \multicolumn{3}{c}{ TS21 } \\
\cline { 2 - 7 } & C10-N6 & C10-N6-C15 & C6-N6-C10-C15 & C10-N6 & N6-C10-C15 & C6-N6-C10-C15 \\
& $\AA$ & deg & deg & $\AA$ & deg & $\operatorname{deg}$ \\
\hline MP2/cc-pVDZ & - & - & - & 2.172 & 94.43 & -90.29 \\
B3PW91/cc-pVDZ & 1.573 & 57.00 & -108.25 & 1.940 & 98.71 & -85.79 \\
B3PW91/6-31+G(d,p) & 1.577 & 56.86 & -108.29 & 1.923 & 99.27 & -84.08 \\
B3LYP/cc-pVDZ & 1.592 & 56.29 & -107.97 & 1.866 & 100.34 & -81.98 \\
B3LYP/6-31+G(d,p) & 1.599 & 56.15 & -108.18 & 1.841 & 101.03 & -81.54 \\
B3LYP/6-31G(d,p) & 1.596 & 56.14 & -107.89 & 1.837 & 100.79 & -80.91 \\
\hline
\end{tabular}

Adenine in the reaction of adenine with 4-biphenylylnitrenium ion at B3LYP/6-31+G(d,p) level

Zero-point correction=

Thermal correction to Energy=

Thermal correction to Enthalpy=

Thermal correction to Gibbs Free Energy=

Sum of electronic and zero-point Energies=

Sum of electronic and thermal Energies=

Sum of electronic and thermal Enthalpies=

Sum of electronic and thermal Free Energies=

$\begin{array}{ccc}\text { Center } & \text { Atomic } & \text { Atomic } \\ \text { Number } & \text { Number } & \text { Type }\end{array}$

0.111549 (Hartree/Particle)

0.119266

0.120210

0.077020

$-467.241601$

$-467.233884$

$-467.232940$

$-467.276130$

$\begin{array}{rrrrrr}1 & 7 & 0 & -0.023752 & 1.923251 & -0.000046 \\ 2 & 1 & 0 & -2.774097 & 1.317353 & 0.000018 \\ 3 & 7 & 0 & -1.182034 & -1.477565 & 0.000041 \\ 4 & 1 & 0 & -3.290352 & -1.192991 & 0.000283 \\ 5 & 6 & 0 & -0.179636 & -0.520724 & -0.000109\end{array}$




$\begin{array}{ccrrrr}6 & 6 & 0 & -0.711366 & 0.773752 & -0.000100 \\ 7 & 7 & 0 & -2.077005 & 0.586836 & -0.000041 \\ 8 & 6 & 0 & 1.295339 & 1.702724 & 0.000223 \\ 9 & 7 & 0 & 1.948344 & 0.527553 & 0.000134 \\ 10 & 6 & 0 & 1.229292 & -0.609968 & -0.000137 \\ 11 & 6 & 0 & -2.291850 & -0.777510 & 0.000148 \\ 12 & 7 & 0 & 1.881688 & -1.796710 & -0.001192 \\ 13 & 1 & 0 & 2.889370 & -1.802352 & 0.003505 \\ 14 & 1 & 0 & 1.366302 & -2.662151 & 0.003695 \\ 15 & 1 & 0 & 1.927419 & 2.586937 & 0.000086\end{array}$

\section{4-biphenylylnitrenium ion in the reaction of adenine with 4-biphenylylnitrenium ion at B3LYP/6-} $31+G(d, p) 1$ eve1

\begin{tabular}{|c|c|c|c|c|c|}
\hline \multicolumn{3}{|c|}{ Zero-point correction $=$} & \multicolumn{3}{|c|}{ 0.186765 (Hartree/Particle) } \\
\hline \multicolumn{3}{|c|}{ Thermal correction to Energy= } & \multicolumn{3}{|c|}{0.196837} \\
\hline \multicolumn{3}{|c|}{ Thermal correction to Enthalpy $=$} & \multicolumn{3}{|l|}{0.197781} \\
\hline \multicolumn{3}{|c|}{ Thermal correction to Gibbs Free Energy= } & \multicolumn{3}{|l|}{0.150541} \\
\hline \multicolumn{3}{|c|}{ Sum of electronic and zero-point Energies= } & \multicolumn{3}{|c|}{-517.603108} \\
\hline \multirow{2}{*}{\multicolumn{3}{|c|}{$\begin{array}{l}\text { Sum of electronic and thermal Energies }= \\
\text { Sum of electronic and thermal Enthalpies= }\end{array}$}} & \multicolumn{3}{|c|}{-517.593036} \\
\hline & & & \multicolumn{3}{|c|}{-517.592092} \\
\hline & \multicolumn{3}{|c|}{-517.639331} \\
\hline \multirow{2}{*}{$\begin{array}{r}\text { Sum of electronic and th } \\
\text { Center } \\
\text { Number }\end{array}$} & \multirow{2}{*}{$\begin{array}{l}\text { Atomic } \\
\text { Number }\end{array}$} & \multirow{2}{*}{$\begin{array}{l}\text { Atomic } \\
\text { Type }\end{array}$} & \multicolumn{3}{|c|}{ Coordinates (Angstroms) } \\
\hline & & & \multicolumn{2}{|c|}{ X $\quad Y$} & Z \\
\hline 1 & 1 & 0 & -0.523652 & -2.140523 & -0.432592 \\
\hline 2 & 6 & 0 & -3.181651 & -0.008514 & -0.001447 \\
\hline 3 & 6 & 0 & -0.328198 & -0.007638 & -0.000723 \\
\hline 4 & 6 & 0 & -2.419617 & -1.230598 & -0.235650 \\
\hline 5 & 6 & 0 & -2.425712 & 1. 219120 & 0.234591 \\
\hline 6 & 6 & 0 & -1.068964 & 1. 210364 & 0.222420 \\
\hline 7 & 6 & 0 & -1.060809 & -1.223373 & -0.222826 \\
\hline 8 & 1 & 0 & -2.989420 & -2.134376 & -0.429951 \\
\hline 9 & 1 & 0 & -2.973480 & 2. 137388 & 0.431836 \\
\hline 10 & 1 & 0 & -0.531776 & 2. 127724 & 0.432022 \\
\hline 11 & 6 & 0 & 1. 110458 & -0.000792 & 0.001305 \\
\hline 12 & 6 & 0 & 1. 844896 & -1.197509 & 0.229265 \\
\hline 13 & 6 & 0 & 1. 836766 & 1. 200774 & -0.227231 \\
\hline 14 & 6 & 0 & 3. 227666 & -1.185332 & 0.242891 \\
\hline 15 & 1 & 0 & 1. 327828 & -2.124967 & 0.448319 \\
\hline 16 & 6 & 0 & 3. 219689 & 1. 197571 & -0.242863 \\
\hline 17 & 1 & 0 & 1. 314394 & 2. 125353 & -0.445922 \\
\hline 18 & 6 & 0 & 3. 919152 & 0.008432 & -0.000393 \\
\hline 19 & 1 & 0 & 3. 775599 & -2.099908 & 0.445046 \\
\hline 20 & 1 & 0 & 3. 761462 & 2. 115638 & -0.445768 \\
\hline 21 & 1 & 0 & 5. 005335 & 0.011997 & -0.000824 \\
\hline 22 & 1 & 0 & -4.921703 & 0.800994 & 0.157731 \\
\hline 23 & 7 & 0 & -4.469520 & -0.102050 & -0.022274 \\
\hline
\end{tabular}


COM1 in the reaction of adenine with 4-biphenylylnitrenium ion at B3LYP/6-31+G(d,p) leve1

Zero-point correction=

Thermal correction to Energy=

Thermal correction to Enthalpy=

Thermal correction to Gibbs Free Energy=

Sum of electronic and zero-point Energies=

Sum of electronic and thermal Energies=

Sum of electronic and thermal Enthalpies=

Sum of electronic and thermal Free Energies=
0. 299909 (Hartree/Particle)

0.319231

0.320175

0. 247107

$-984.853438$

$-984.834115$

$-984.833171$

$-984.906239$

\begin{tabular}{|c|c|c|c|c|c|}
\hline \multirow{2}{*}{$\begin{array}{l}\text { Center } \\
\text { Number }\end{array}$} & \multirow{2}{*}{$\begin{array}{l}\text { Atomic } \\
\text { Number }\end{array}$} & \multirow{2}{*}{$\begin{array}{l}\text { Atomic } \\
\text { Type }\end{array}$} & \multicolumn{3}{|c|}{ Coordinates (Angstroms) } \\
\hline & & & X & $\mathrm{Y}$ & Z \\
\hline 1 & 7 & 0 & 0.445306 & -0.989214 & -1.505462 \\
\hline 2 & 1 & 0 & -0.451184 & -1.592193 & 1. 105008 \\
\hline 3 & 7 & 0 & 2.585620 & -0.551563 & 1. 339682 \\
\hline 4 & 1 & 0 & 1. 307807 & -1.189036 & 2.918846 \\
\hline 5 & 6 & 0 & 2. 317225 & -0.487825 & -0.013437 \\
\hline 6 & 7 & 0 & 7. 866328 & -1.262138 & -1.093505 \\
\hline 7 & 6 & 0 & 1. 015263 & -0.929699 & -0.298711 \\
\hline 8 & 7 & 0 & 0.489128 & -1.264602 & 0.928848 \\
\hline 9 & 6 & 0 & 1. 267097 & -0.556713 & -2.468625 \\
\hline 10 & 7 & 0 & 2.527440 & -0.100090 & -2.345282 \\
\hline 11 & 6 & 0 & 3. 069562 & -0.038547 & -1.116113 \\
\hline 12 & 1 & 0 & 5. 056744 & -2.259924 & -4.532022 \\
\hline 13 & 6 & 0 & 1. 471336 & -1.014085 & 1. 864026 \\
\hline 14 & 1 & 0 & 8. 446821 & -0.798066 & -1.801441 \\
\hline 15 & 6 & 0 & 6. 786618 & -1.756403 & -1.607613 \\
\hline 16 & 6 & 0 & 4. 449707 & -3.083586 & -2.597536 \\
\hline 17 & 6 & 0 & 6. 407011 & -1.679286 & -3.019089 \\
\hline 18 & 6 & 0 & 5. 881635 & -2.481982 & -0.717940 \\
\hline 19 & 6 & 0 & 4. 788727 & -3.134968 & -1.203232 \\
\hline 20 & 6 & 0 & 5. 290849 & -2.304108 & -3.476095 \\
\hline 21 & 1 & 0 & 7. 054367 & -1.133086 & -3.700237 \\
\hline 22 & 1 & 0 & 6. 136361 & -2.498297 & 0.336984 \\
\hline 23 & 1 & 0 & 4. 141399 & -3.664325 & -0.515777 \\
\hline 24 & 7 & 0 & 4. 350504 & 0.424169 & -0.986411 \\
\hline 25 & 1 & 0 & 4. 682450 & 1. 001845 & -1.748126 \\
\hline 26 & 1 & 0 & 4. 639402 & 0.708126 & -0.058124 \\
\hline 27 & 1 & 0 & 0.867376 & -0.567097 & -3.478684 \\
\hline 28 & 6 & 0 & 3. 312901 & -3.808309 & -3.121359 \\
\hline 29 & 6 & 0 & 2. 721082 & -3.448276 & -4.364201 \\
\hline 30 & 6 & 0 & 2. 762970 & -4.913545 & -2.413899 \\
\hline 31 & 6 & 0 & 1. 637930 & -4.154154 & -4.865641 \\
\hline 32 & 1 & 0 & 3. 081524 & -2.585492 & -4.911253 \\
\hline 33 & 6 & 0 & 1. 695180 & -5.629111 & -2.933647 \\
\hline 34 & 1 & 0 & 3. 208026 & -5.241669 & -1.482021 \\
\hline 35 & 6 & 0 & 1. 125696 & -5.250041 & -4.157588 \\
\hline 36 & 1 & 0 & 1. 185546 & -3.853951 & -5.804928 \\
\hline 37 & 1 & 0 & 1. 305204 & -6.486092 & -2.394701 \\
\hline 38 & 1 & 0 & 0.284615 & -5.807302 & -4.558444 \\
\hline
\end{tabular}




\section{S-TS1 in the reaction of adenine with 4-biphenylylnitrenium ion at B3LYP/6-31+G(d,p) leve1}

Zero-point correction=

Thermal correction to Energy=

Thermal correction to Enthalpy=

Thermal correction to Gibbs Free Energy=

Sum of electronic and zero-point Energies=

Sum of electronic and thermal Energies=

Sum of electronic and thermal Enthalpies=

Sum of electronic and thermal Free Energies=
0.301451 (Hartree/Particle)

0.319232

0.320176

0.252400

$-984.843272$

$-984.825491$

$-984.824547$

$-984.892323$

\begin{tabular}{|c|c|c|c|c|c|}
\hline \multirow{2}{*}{$\begin{array}{l}\text { Center } \\
\text { Number }\end{array}$} & \multirow{2}{*}{$\begin{array}{l}\text { Atomic } \\
\text { Number }\end{array}$} & \multirow{2}{*}{$\begin{array}{l}\text { Atomic } \\
\text { Type }\end{array}$} & \multicolumn{3}{|c|}{ Coordinates (Angstroms) } \\
\hline & & & $X$ & $\mathrm{Y}$ & Z \\
\hline 1 & 7 & 0 & 3. 114444 & -2.535286 & 0.844083 \\
\hline 2 & 1 & 0 & 5.278724 & -2.385420 & -0.969131 \\
\hline 3 & 7 & 0 & 3.875306 & 0.514229 & -0.878857 \\
\hline 4 & 1 & 0 & 5.556186 & -0.080069 & -2.039012 \\
\hline 5 & 6 & 0 & 3. 133032 & -0.238749 & 0.005586 \\
\hline 6 & 7 & 0 & 0.534928 & 3. 979089 & -0.320396 \\
\hline 7 & 6 & 0 & 3. 615611 & -1.564897 & 0.086566 \\
\hline 8 & 7 & 0 & 4. 682140 & -1.590277 & -0.779542 \\
\hline 9 & 6 & 0 & 2. 062503 & -2.140174 & 1. 570699 \\
\hline 10 & 7 & 0 & 1. 506670 & -0.911424 & 1. 598570 \\
\hline 11 & 6 & 0 & 2. 043143 & 0.034403 & 0.831041 \\
\hline 12 & 1 & 0 & -3.456599 & 2. 135419 & 0.866945 \\
\hline 13 & 6 & 0 & 4. 785823 & -0.326392 & -1.320528 \\
\hline 14 & 1 & 0 & 0.158402 & 4. 873783 & 0. 008789 \\
\hline 15 & 6 & 0 & -0.309387 & 3. 019675 & -0.173949 \\
\hline 16 & 6 & 0 & -2.082881 & 0.751314 & -0.153883 \\
\hline 17 & 6 & 0 & -1.644054 & 3. 111764 & 0.422231 \\
\hline 18 & 6 & 0 & 0.139747 & 1. 660585 & -0.615656 \\
\hline 19 & 6 & 0 & -0.810794 & 0.601526 & -0.670393 \\
\hline 20 & 6 & 0 & -2.461893 & 2. 036764 & 0.447852 \\
\hline 21 & 1 & 0 & -1.962173 & 4. 070365 & 0.822718 \\
\hline 22 & 1 & 0 & 0.949112 & 1. 670253 & -1.339956 \\
\hline 23 & 1 & 0 & -0.508452 & -0.341131 & -1.111026 \\
\hline 24 & 7 & 0 & 1. 425444 & 1. 310253 & 0.858744 \\
\hline 25 & 1 & 0 & 0.893042 & 1. 430309 & 1. 720901 \\
\hline 26 & 1 & 0 & 2. 067771 & 2. 088889 & 0.695879 \\
\hline 27 & 1 & 0 & 1. 605021 & -2.885324 & 2. 213123 \\
\hline 28 & 6 & 0 & -3.086335 & -0.317030 & -0.236602 \\
\hline 29 & 6 & 0 & -4.098621 & -0.438182 & 0.741465 \\
\hline 30 & 6 & 0 & -3.054781 & -1.257264 & -1.291018 \\
\hline 31 & 6 & 0 & -5.025746 & -1.475257 & 0.679508 \\
\hline 32 & 1 & 0 & -4.139717 & 0.251010 & 1. 578257 \\
\hline 33 & 6 & 0 & -3.995442 & -2.278940 & -1.360101 \\
\hline 34 & 1 & 0 & -2.319770 & -1.159370 & -2.083093 \\
\hline 35 & 6 & 0 & -4.980878 & -2.395232 & -0.372320 \\
\hline 36 & 1 & 0 & -5.783748 & -1.565432 & 1. 450845 \\
\hline 37 & 1 & 0 & -3.970645 & -2.978978 & -2.189052 \\
\hline 38 & 1 & 0 & -5.713962 & -3.193880 & -0.427393 \\
\hline
\end{tabular}




\section{S-INT1 in the reaction of adenine with 4-biphenylylnitrenium ion at B3LYP/6-31+G(d,p) leve1}

Zero-point correction=

Thermal correction to Energy=

Thermal correction to Enthalpy=

Thermal correction to Gibbs Free Energy=

Sum of electronic and zero-point Energies=

Sum of electronic and thermal Energies=

Sum of electronic and thermal Enthalpies=

Sum of electronic and thermal Free Energies=
0. 303282 (Hartree/Particle)

0.320774

0.321718

0. 255381

$-984.856850$

$-984.839358$

$-984.838414$

$-984.904751$

\begin{tabular}{|c|c|c|c|c|c|}
\hline \multirow{2}{*}{$\begin{array}{l}\text { Center } \\
\text { Number }\end{array}$} & \multirow{2}{*}{$\begin{array}{l}\text { Atomic } \\
\text { Number }\end{array}$} & \multirow{2}{*}{$\begin{array}{l}\text { Atomic } \\
\text { Type }\end{array}$} & \multicolumn{3}{|c|}{ Coordinates (Angstroms) } \\
\hline & & & $X$ & Y & Z \\
\hline 1 & 7 & 0 & -4.234992 & -2.052061 & -0.733794 \\
\hline 2 & 1 & 0 & -5.403961 & -1.652807 & 1. 801614 \\
\hline 3 & 7 & 0 & -3.081352 & 0.552586 & 1. 443228 \\
\hline 4 & 1 & 0 & -4.358590 & 0.271893 & 3. 119551 \\
\hline 5 & 6 & 0 & -3.044991 & -0.155514 & 0.260134 \\
\hline 6 & 7 & 0 & -0.738617 & 3. 376861 & -0.645145 \\
\hline 7 & 6 & 0 & -4.015549 & -1.189631 & 0.251055 \\
\hline 8 & 7 & 0 & -4.643653 & -1.074442 & 1. 466194 \\
\hline 9 & 6 & 0 & -3.428089 & -1.873780 & -1.783238 \\
\hline 10 & 7 & 0 & -2.467889 & -0.930986 & -1.907704 \\
\hline 11 & 6 & 0 & -2.290331 & -0.088999 & -0.904068 \\
\hline 12 & 1 & 0 & 3.445097 & 2. 296901 & 0.766195 \\
\hline 13 & 6 & 0 & -4.044652 & -0.025312 & 2. 127767 \\
\hline 14 & 1 & 0 & -0.514142 & 4. 371888 & -0.657330 \\
\hline 15 & 6 & 0 & 0.169806 & 2. 622330 & -0.145763 \\
\hline 16 & 6 & 0 & 2. 212605 & 0.592805 & 0.131488 \\
\hline 17 & 6 & 0 & 1. 496585 & 2. 973251 & 0.338342 \\
\hline 18 & 6 & 0 & -0.194191 & 1. 137472 & -0.065584 \\
\hline 19 & 6 & 0 & 0.956381 & 0.185576 & -0.171811 \\
\hline 20 & 6 & 0 & 2. 446041 & 2. 015383 & 0.448571 \\
\hline 21 & 1 & 0 & 1. 725632 & 4. 019090 & 0.519841 \\
\hline 22 & 1 & 0 & -0.717929 & 0.996839 & 0.892224 \\
\hline 23 & 1 & 0 & 0.741679 & -0.854549 & -0.400262 \\
\hline 24 & 7 & 0 & -1.265992 & 0.934176 & -1.131530 \\
\hline 25 & 1 & 0 & -0.831758 & 0.711234 & -2.036276 \\
\hline 26 & 1 & 0 & -1.655339 & 1. 920453 & -1.209574 \\
\hline 27 & 1 & 0 & -3.550112 & -2.548074 & -2.623841 \\
\hline 28 & 6 & 0 & 3. 362521 & -0.342711 & 0.168965 \\
\hline 29 & 6 & 0 & 4. 624362 & 0.058519 & -0.306342 \\
\hline 30 & 6 & 0 & 3. 212159 & -1.649900 & 0.665501 \\
\hline 31 & 6 & 0 & 5. 699256 & -0.830500 & -0.303541 \\
\hline 32 & 1 & 0 & 4. 764468 & 1. 057617 & -0.709090 \\
\hline 33 & 6 & 0 & 4. 289956 & -2.534765 & 0.674012 \\
\hline 34 & 1 & 0 & 2. 258031 & -1.964231 & 1. 078282 \\
\hline 35 & 6 & 0 & 5. 535632 & -2.128663 & 0.187145 \\
\hline 36 & 1 & 0 & 6. 663129 & -0.509663 & -0.686046 \\
\hline 37 & 1 & 0 & 4. 161096 & -3.536229 & 1. 072717 \\
\hline
\end{tabular}


TS2 in the reaction of adenine with 4-biphenylylnitrenium ion at B3LYP/6-31+G(d,p) leve1

Zero-point correction=

Thermal correction to Energy=

Thermal correction to Enthalpy=

Thermal correction to Gibbs Free Energy=

Sum of electronic and zero-point Energies=

Sum of electronic and thermal Energies=

Sum of electronic and thermal Enthalpies=

Sum of electronic and thermal Free Energies=

\author{
0. 299427 (Hartree/Particle) \\ 0.316520 \\ 0.317464 \\ 0.252340 \\ $-984.858611$ \\ $-984.841518$ \\ $-984.840574$ \\ $-984.905697$
}

\begin{tabular}{|c|c|c|c|c|c|}
\hline \multirow{2}{*}{$\begin{array}{l}\text { Center } \\
\text { Number }\end{array}$} & \multirow{2}{*}{$\begin{array}{l}\text { Atomic } \\
\text { Number }\end{array}$} & \multirow{2}{*}{$\begin{array}{l}\text { Atomic } \\
\text { Type }\end{array}$} & \multicolumn{3}{|c|}{ Coordinates (Angstroms) } \\
\hline & & & $X$ & $\mathrm{Y}$ & Z \\
\hline 1 & 7 & 0 & -4.572655 & -1.779160 & -0.730474 \\
\hline 2 & 1 & 0 & -5.425856 & -1.515718 & 1.941423 \\
\hline 3 & 7 & 0 & -2.849565 & 0.378117 & 1. 557385 \\
\hline 4 & 1 & 0 & -3.993490 & 0.085912 & 3. 325952 \\
\hline 5 & 6 & 0 & -3.025783 & -0.190724 & 0.309858 \\
\hline 6 & 7 & 0 & -0.838461 & 3. 116273 & -0.651883 \\
\hline 7 & 6 & 0 & -4.133199 & -1.071948 & 0.304725 \\
\hline 8 & 7 & 0 & -4.623478 & -1.010970 & 1.586525 \\
\hline 9 & 6 & 0 & -3.847106 & -1.592814 & -1.836031 \\
\hline 10 & 7 & 0 & -2.771528 & -0.787235 & -1.969793 \\
\hline 11 & 6 & 0 & -2.370745 & -0.092333 & -0.915071 \\
\hline 12 & 1 & 0 & 3. 356387 & 2. 271126 & 0.876228 \\
\hline 13 & 6 & 0 & -3.819297 & -0.136322 & 2. 281666 \\
\hline 14 & 1 & 0 & -0.786336 & 4. 131364 & -0.679496 \\
\hline 15 & 6 & 0 & 0.115654 & 2. 441633 & -0.112489 \\
\hline 16 & 6 & 0 & 2. 255359 & 0.538034 & 0.106911 \\
\hline 17 & 6 & 0 & 1. 383377 & 2. 862696 & 0.434031 \\
\hline 18 & 6 & 0 & -0.172728 & 0.940975 & -0.116978 \\
\hline 19 & 6 & 0 & 1. 036005 & 0.070395 & -0.259368 \\
\hline 20 & 6 & 0 & 2. 384218 & 1. 948346 & 0.516062 \\
\hline 21 & 1 & 0 & 1. 549542 & 3. 908710 & 0.670848 \\
\hline 22 & 1 & 0 & -0.654853 & 0.733002 & 0.852879 \\
\hline 23 & 1 & 0 & 0.893164 & -0.965245 & -0.553681 \\
\hline 24 & 7 & 0 & -1.254724 & 0.797696 & -1.156043 \\
\hline 25 & 1 & 0 & -0.870429 & 0.552445 & -2.074548 \\
\hline 26 & 1 & 0 & -1.504594 & 1. 980134 & -1.167835 \\
\hline 27 & 1 & 0 & -4.145601 & -2.143046 & -2.721948 \\
\hline 28 & 6 & 0 & 3. 464977 & -0.321082 & 0.124193 \\
\hline 29 & 6 & 0 & 4. 705479 & 0.182500 & -0.307192 \\
\hline 30 & 6 & 0 & 3. 390728 & -1.656920 & 0.556667 \\
\hline 31 & 6 & 0 & 5. 835910 & -0.634687 & -0.322778 \\
\hline 32 & 1 & 0 & 4. 787117 & 1. 206006 & -0.662599 \\
\hline 33 & 6 & 0 & 4. 523662 & -2.470292 & 0.546101 \\
\hline 34 & 1 & 0 & 2. 451479 & -2.051498 & 0.933241 \\
\hline 35 & 6 & 0 & 5. 748442 & -1.962461 & 0.104163 \\
\hline 36 & 1 & 0 & 6. 783219 & -0.235188 & -0.671046 \\
\hline 37 & 1 & 0 & 4. 452918 & -3.495959 & 0.894589 \\
\hline
\end{tabular}


INT2 in the reaction of adenine with 4-biphenylylnitrenium ion at B3LYP/6-31+G(d,p) 1 eve1

Zero-point correction=

Thermal correction to Energy=

Thermal correction to Enthalpy=

Thermal correction to Gibbs Free Energy=

Sum of electronic and zero-point Energies=

Sum of electronic and thermal Energies=

Sum of electronic and thermal Enthalpies=

Sum of electronic and thermal Free Energies=

\begin{tabular}{|c|c|c|c|c|c|}
\hline \multirow{2}{*}{$\begin{array}{l}\text { Center } \\
\text { Number }\end{array}$} & \multirow{2}{*}{$\begin{array}{l}\text { Atomic } \\
\text { Number }\end{array}$} & \multirow{2}{*}{$\begin{array}{l}\text { Atomic } \\
\text { Type }\end{array}$} & \multicolumn{3}{|c|}{ Coordinates (Angstroms) } \\
\hline & & & $X$ & $\mathrm{Y}$ & Z \\
\hline 1 & 7 & 0 & -4.619705 & -1.597827 & -0.802711 \\
\hline 2 & 1 & 0 & -4.940025 & -2.052937 & 1. 957752 \\
\hline 3 & 7 & 0 & -2.389608 & -0.132943 & 1. 593332 \\
\hline 4 & 1 & 0 & -3.198301 & -0.880065 & 3. 415940 \\
\hline 5 & 6 & 0 & -2.821815 & -0.354446 & 0.293178 \\
\hline 6 & 7 & 0 & -0.889226 & 3. 426584 & -0.009652 \\
\hline 7 & 6 & 0 & -3.949878 & -1.191548 & 0.275373 \\
\hline 8 & 7 & 0 & -4.191070 & -1.473263 & 1. 602063 \\
\hline 9 & 6 & 0 & -4.093793 & -1.116605 & -1.932530 \\
\hline 10 & 7 & 0 & -3.019210 & -0.313209 & -2.063275 \\
\hline 11 & 6 & 0 & -2.374166 & 0.082783 & -0.963880 \\
\hline 12 & 1 & 0 & 3. 331517 & 2. 193105 & 0.987631 \\
\hline 13 & 6 & 0 & -3.231797 & -0.818193 & 2. 336860 \\
\hline 14 & 1 & 0 & -0.773169 & 4. 394831 & 0.262104 \\
\hline 15 & 6 & 0 & 0.068265 & 2. 534269 & 0.148356 \\
\hline 16 & 6 & 0 & 2. 189137 & 0.571770 & 0.087229 \\
\hline 17 & 6 & 0 & 1. 353969 & 2. 856533 & 0.654524 \\
\hline 18 & 6 & 0 & -0.249483 & 1. 095723 & -0.156069 \\
\hline 19 & 6 & 0 & 0.945392 & 0.210974 & -0.333035 \\
\hline 20 & 6 & 0 & 2. 349669 & 1. 913959 & 0.615522 \\
\hline 21 & 1 & 0 & 1. 560673 & 3. 863452 & 1. 004110 \\
\hline 22 & 1 & 0 & -0.687024 & 0.761548 & 0.820248 \\
\hline 23 & 1 & 0 & 0.752340 & -0.774294 & -0.746825 \\
\hline 24 & 7 & 0 & -1.307835 & 0.961013 & -1.146239 \\
\hline 25 & 1 & 0 & -1.018277 & 1. 003596 & -2.119989 \\
\hline 26 & 1 & 0 & -1.759162 & 3. 139571 & -0.449254 \\
\hline 27 & 1 & 0 & -4.582196 & -1.405169 & -2.858071 \\
\hline 28 & 6 & 0 & 3. 360052 & -0.338763 & 0.019737 \\
\hline 29 & 6 & 0 & 4. 619548 & 0.143866 & -0.376396 \\
\hline 30 & 6 & 0 & 3. 223656 & -1.700404 & 0.339397 \\
\hline 31 & 6 & 0 & 5. 713092 & -0.718311 & -0.461184 \\
\hline 32 & 1 & 0 & 4. 745156 & 1. 187859 & -0.651298 \\
\hline 33 & 6 & 0 & 4. 319321 & -2.559816 & 0.256415 \\
\hline 34 & 1 & 0 & 2. 266607 & -2.083071 & 0.682425 \\
\hline 35 & 6 & 0 & 5.565656 & -2.071562 & -0.144989 \\
\hline
\end{tabular}

0. 302909 (Hartree/Particle)

0.320606

0. 321550

0. 255149

$-984.886858$

$-984.869161$

$-984.868217$

$-984.934617$ 


\begin{tabular}{rrrrrr}
36 & 1 & 0 & 6.677109 & -0.334542 & -0.779888 \\
37 & 1 & 0 & 4.202200 & -3.607207 & 0.516264 \\
38 & 1 & 0 & 6.418179 & -2.740527 & -0.206847 \\
\hline
\end{tabular}

TS3 in the reaction of adenine with 4-biphenylylnitrenium ion at B3LYP/6-31+G(d,p) leve1

Zero-point correction=

Thermal correction to Energy=

Thermal correction to Enthalpy=

Thermal correction to Gibbs Free Energy=

Sum of electronic and zero-point Energies=

Sum of electronic and thermal Energies=

Sum of electronic and thermal Enthalpies=

Sum of electronic and thermal Free Energies=

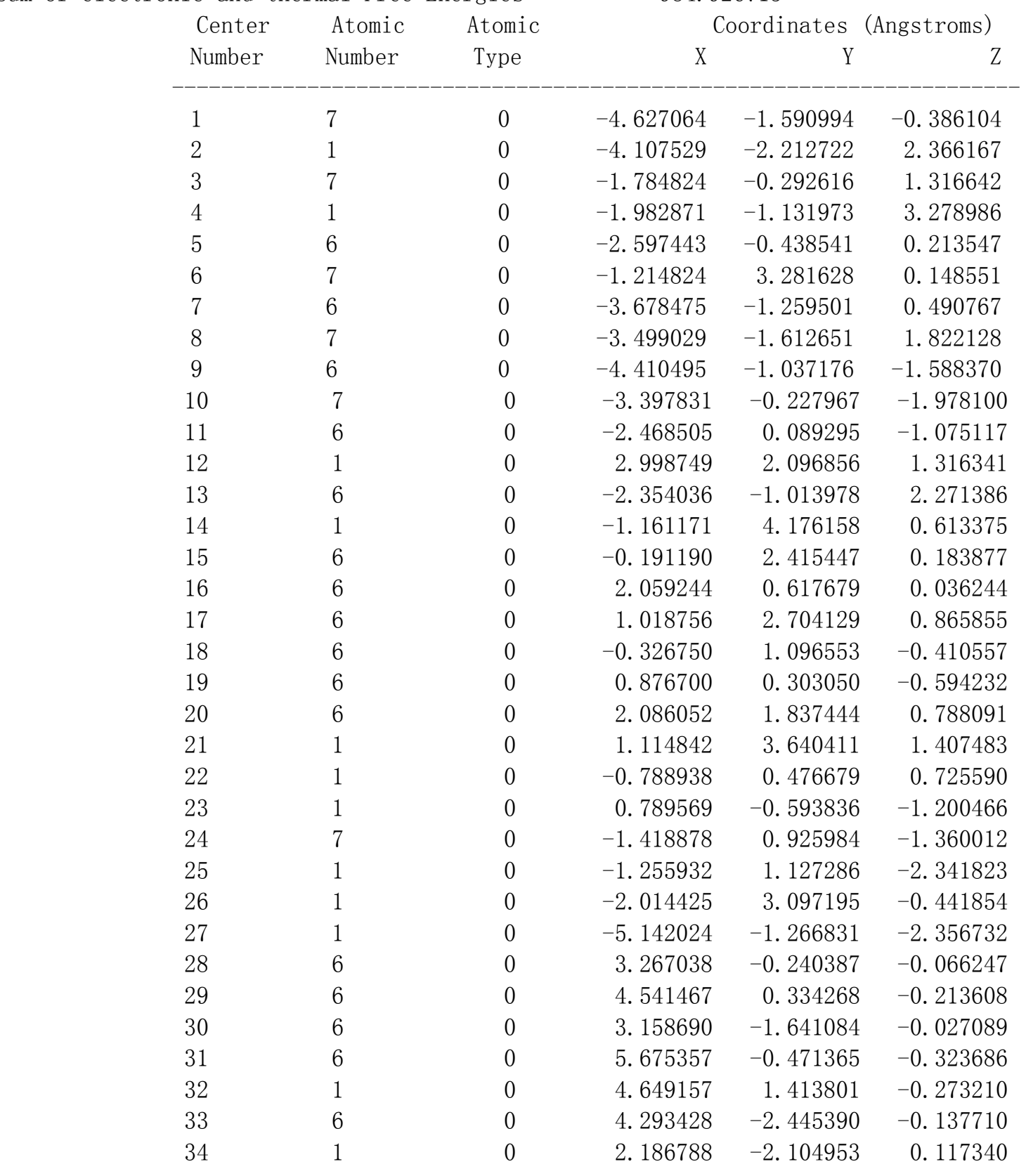

0. 297939 (Hartree/Particle)

0.315339

0.316283

0. 251714

$-984.880489$

$-984.863088$

$-984.862144$

$-984.926713$ 


$\begin{array}{llllll}35 & 6 & 0 & 5.554947 & -1.863014 & -0.286102 \\ 36 & 1 & 0 & 6.650997 & -0.011759 & -0.447490 \\ 37 & 1 & 0 & 4.193436 & -3.525682 & -0.097501 \\ 38 & 1 & 0 & 6.437789 & -2.488877 & -0.370218\end{array}$

\section{INT3 in the reaction of adenine with 4-biphenylylnitrenium ion at B3LYP/6-31+G(d,p) level}

Zero-point correction=

Thermal correction to Energy=

Thermal correction to Enthalpy=

Thermal correction to Gibbs Free Energy=

Sum of electronic and zero-point Energies=

Sum of electronic and thermal Energies=

Sum of electronic and thermal Enthalpies=

Sum of electronic and thermal Free Energies=
0. 303973 (Hartree/Particle)

0. 321747

0. 322692

0. 256514

$-984.916578$

$-984.898804$

$-984.897859$

$-984.964037$

\begin{tabular}{|c|c|c|c|c|c|}
\hline \multirow{2}{*}{$\begin{array}{l}\text { Center } \\
\text { Number }\end{array}$} & \multirow{2}{*}{$\begin{array}{l}\text { Atomic } \\
\text { Number }\end{array}$} & \multirow{2}{*}{$\begin{array}{l}\text { Atomic } \\
\text { Type }\end{array}$} & \multicolumn{3}{|c|}{ Coordinates (Angstroms) } \\
\hline & & & X & Y & Z \\
\hline 1 & 7 & 0 & -4.680005 & -1.553636 & -0.179286 \\
\hline 2 & 1 & 0 & -4.611301 & -1.315438 & 2. 592223 \\
\hline 3 & 7 & 0 & -2.050549 & 0.135874 & 1. 455982 \\
\hline 4 & 1 & 0 & -2.601087 & -0.024600 & 3.522905 \\
\hline 5 & 6 & 0 & -2.637200 & -0.306151 & 0.272022 \\
\hline 6 & 7 & 0 & -1.455646 & 2. 861985 & -0.329794 \\
\hline 7 & 6 & 0 & -3.807589 & -0.977291 & 0.633984 \\
\hline 8 & 7 & 0 & -3.872146 & -0.913856 & 2. 025495 \\
\hline 9 & 6 & 0 & -4.316792 & -1.419238 & -1.464590 \\
\hline 10 & 7 & 0 & -3.228525 & -0.809705 & -1.949406 \\
\hline 11 & 6 & 0 & -2.342206 & -0.238048 & -1.115788 \\
\hline 12 & 1 & 0 & 2. 825901 & 2. 217620 & 1. 173712 \\
\hline 13 & 6 & 0 & -2.808711 & -0.236627 & 2. 484493 \\
\hline 14 & 1 & 0 & -1.368027 & 3. 843519 & -0.096907 \\
\hline 15 & 6 & 0 & -0.262053 & 2. 137209 & -0.268840 \\
\hline 16 & 6 & 0 & 2. 142390 & 0.593724 & -0.074979 \\
\hline 17 & 6 & 0 & 0.836313 & 2.587076 & 0.487661 \\
\hline 18 & 6 & 0 & -0.147123 & 0.872710 & -0.886656 \\
\hline 19 & 6 & 0 & 1. 035855 & 0.131118 & -0.801467 \\
\hline 20 & 6 & 0 & 2. 003888 & 1. 834996 & 0.577155 \\
\hline 21 & 1 & 0 & 0.775689 & 3. 548259 & 0.991540 \\
\hline 22 & 1 & 0 & -1.204434 & 0.696603 & 1. 517016 \\
\hline 23 & 1 & 0 & 1. 090646 & -0.814418 & -1.332799 \\
\hline 24 & 7 & 0 & -1.251842 & 0.359149 & -1.648784 \\
\hline 25 & 1 & 0 & -1.154483 & 0.231861 & -2.651805 \\
\hline 26 & 1 & 0 & -1.995827 & 2. 730123 & -1.177160 \\
\hline 27 & 1 & 0 & -4.981980 & -1.859351 & -2.201151 \\
\hline 28 & 6 & 0 & 3. 400745 & -0.191322 & 0.002641 \\
\hline 29 & 6 & 0 & 4. 649750 & 0.453896 & -0.026363 \\
\hline 30 & 6 & 0 & 3. 374528 & -1.593828 & 0.100445 \\
\hline 31 & 6 & 0 & 5. 834172 & -0.280883 & 0.040651 \\
\hline
\end{tabular}




$\begin{array}{rrrrrr}32 & 1 & 0 & 4.698101 & 1.533825 & -0.131832 \\ 33 & 6 & 0 & 4.559182 & -2.328274 & 0.166168 \\ 34 & 1 & 0 & 2.422417 & -2.115291 & 0.149845 \\ 35 & 6 & 0 & 5.793724 & -1.674262 & 0.137142 \\ 36 & 1 & 0 & 6.788777 & 0.235268 & 0.006422 \\ 37 & 1 & 0 & 4.517831 & -3.410233 & 0.248408 \\ 38 & 1 & 0 & 6.715540 & -2.245258 & 0.188063\end{array}$

\section{Adenine in the reaction of adenine with 4-biphenylylnitrenium ion at B3PW91/6-31+G(d,p) level}

Zero-point correction=

Thermal correction to Energy=

Thermal correction to Enthalpy=

Thermal correction to Gibbs Free Energy=

Sum of electronic and zero-point Energies=

Sum of electronic and thermal Energies=

Sum of electronic and thermal Enthalpies=

Sum of electronic and thermal Free Energies=

\begin{tabular}{cccccr}
$\begin{array}{c}\text { Center } \\
\text { Number }\end{array}$ & $\begin{array}{c}\text { Atomic } \\
\text { Number }\end{array}$ & $\begin{array}{c}\text { Atomic } \\
\text { Type }\end{array}$ & \multicolumn{3}{c}{ Coordinates } \\
X Angstroms) & Y & $Z$ \\
\hline 1 & 7 & 0 & 0.026718 & 1.920048 & -0.000053 \\
2 & 1 & 0 & 2.768004 & 1.312524 & 0.000238 \\
3 & 7 & 0 & 1.177686 & -1.476207 & -0.000094 \\
4 & 1 & 0 & 3.283712 & -1.191790 & 0.000345 \\
5 & 6 & 0 & 0.180034 & -0.521752 & -0.000184 \\
6 & 6 & 0 & 0.710005 & 0.771307 & -0.000161 \\
7 & 7 & 0 & 2.071090 & 0.583309 & 0.000132 \\
8 & 6 & 0 & -1.288958 & 1.698556 & 0.000057 \\
9 & 7 & 0 & -1.943020 & 0.527873 & 0.000175 \\
10 & 6 & 0 & -1.227320 & -0.607870 & -0.000106 \\
11 & 6 & 0 & 2.284896 & -0.775784 & 0.000149 \\
12 & 7 & 0 & -1.879641 & -1.789762 & -0.001446 \\
13 & 1 & 0 & -2.886234 & -1.792953 & 0.004804 \\
14 & 1 & 0 & -1.366127 & -2.655020 & 0.004595 \\
15 & 1 & 0 & -1.921122 & 2.583677 & 0.000485
\end{tabular}

0.112412 (Hartree/Particle)

0.119954

0.120898

0.079981

$-467.062369$

$-467.054828$

$-467.053883$

$-467.094800$

4-biphenylylnitrenium ion in the reaction of adenine with 4-biphenylylnitrenium ion at B3PW91/6$31+\mathrm{G}(\mathrm{d}, \mathrm{p})$ level

Zero-point correction=

Thermal correction to Energy=

Thermal correction to Enthalpy=

Thermal correction to Gibbs Free Energy=

Sum of electronic and zero-point Energies=

Sum of electronic and thermal Energies=

Sum of electronic and thermal Enthalpies=

Sum of electronic and thermal Free Energies=

$$
\text { Center Atomic Atomic }
$$

0.187095 (Hartree/Particle)

0.197208

0.198152

0.150829

$-517.394106$

$-517.383993$

$-517.383049$

$-517.430371$

Coordinates (Angstroms) 


$\begin{array}{rlrrrr}\text { Number } & \text { Number } & \text { Type } & \text { X } & \text { Y } \\ ------1 & & \\ 1 & 6 & 0 & 3.919108 & 0.008252 & 0.000181 \\ 2 & 6 & 0 & 3.219869 & 1.197473 & -0.242616 \\ 3 & 6 & 0 & 1.837012 & 1.200793 & -0.227416 \\ 4 & 6 & 0 & 1.110415 & -0.000692 & 0.000794 \\ 5 & 6 & 0 & 1.844620 & -1.197496 & 0.229034 \\ 6 & 6 & 0 & 3.227403 & -1.185430 & 0.243206 \\ 7 & 6 & 0 & -0.328212 & -0.007379 & -0.001367 \\ 8 & 6 & 0 & -1.060832 & -1.223262 & -0.223200 \\ 9 & 6 & 0 & -2.419568 & -1.230689 & -0.235636 \\ 10 & 6 & 0 & -3.181607 & -0.008594 & -0.001339 \\ 11 & 6 & 0 & -2.425706 & 1.219056 & 0.234570 \\ 12 & 6 & 0 & -1.068914 & 1.210493 & 0.222083 \\ 13 & 7 & 0 & -4.469476 & -0.101999 & -0.021646 \\ 14 & 1 & 0 & -0.531828 & 2.127899 & 0.431715 \\ 15 & 1 & 0 & -4.921436 & 0.801057 & 0.158796 \\ 16 & 1 & 0 & -2.973542 & 2.137243 & 0.432075 \\ 17 & 1 & 0 & -2.989380 & -2.134501 & -0.429735 \\ 18 & 1 & 0 & -0.523459 & -2.140304 & -0.432893 \\ 19 & 1 & 0 & 1.314730 & 2.125301 & -0.446644 \\ 20 & 1 & 0 & 1.327442 & -2.124935 & 0.447920 \\ 21 & 1 & 0 & 3.761782 & 2.115519 & -0.445252 \\ 22 & 1 & 0 & 3.775211 & -2.100059 & 0.445460 \\ 23 & 1 & 0 & 5.005289 & 0.011607 & 0.000326\end{array}$

COM1 in the reaction of adenine with 4-biphenylylnitrenium ion at B3PW91/6-31+G(d,p) leve1

Zero-point correction $=$

Thermal correction to Energy=

Thermal correction to Enthalpy=

Thermal correction to Gibbs Free Energy=

Sum of electronic and zero-point Energies=

Sum of electronic and thermal Energies=

Sum of electronic and thermal Enthalpies=

Sum of electronic and thermal Free Energies=
0. 301002 (Hartree/Particle)

0. 320270

0.321215

0.249004

$-984.464873$

$-984.445605$

$-984.444661$

$-984.516871$

Coordinates (Angstroms)

X Y Y

\begin{tabular}{lccccc} 
Number & Number & Type & \multicolumn{2}{c}{ X } & Y \\
\hline 1 & 7 & 0 & 0.543119 & -2.806792 & 0.994886 \\
2 & 1 & 0 & 1.883855 & -4.153026 & -1.084188 \\
3 & 7 & 0 & 3.124240 & -1.195381 & -0.890582 \\
4 & 1 & 0 & 3.660914 & -2.739966 & -2.250737 \\
5 & 6 & 0 & 2.171908 & -1.220024 & 0.101505 \\
6 & 7 & 0 & 1.760429 & 4.306127 & -0.527513 \\
7 & 6 & 0 & 1.510308 & -2.455986 & 0.148341 \\
8 & 7 & 0 & 2.093527 & -3.192206 & -0.852711 \\
9 & 6 & 0 & 0.249687 & -1.822362 & 1.847845 \\
10 & 7 & 0 & 0.796948 & -0.599212 & 1.929829 \\
11 & 6 & 0 & 1.780774 & -0.281827 & 1.074022 \\
12 & 1 & 0 & -1.802153 & 2.351150 & 1.515839 \\
13 & 6 & 0 & 3.046975 & -2.390334 & -1.431012
\end{tabular}




$\begin{array}{rrrrrr}14 & 1 & 0 & 1.679407 & 5.027980 & 0.196119 \\ 15 & 6 & 0 & 0.883492 & 3.371001 & -0.364727 \\ 16 & 6 & 0 & -1.121122 & 1.338785 & -0.296512 \\ 17 & 6 & 0 & -0.109152 & 3.318433 & 0.705617 \\ 18 & 6 & 0 & 0.851972 & 2.295454 & -1.349174 \\ 19 & 6 & 0 & -0.126770 & 1.349591 & -1.326474 \\ 20 & 6 & 0 & -1.052273 & 2.342826 & 0.733856 \\ 21 & 1 & 0 & -0.097691 & 4.099138 & 1.462606 \\ 22 & 1 & 0 & 1.623288 & 2.304682 & -2.113195 \\ 23 & 1 & 0 & -0.125572 & 0.565934 & -2.074776 \\ 24 & 7 & 0 & 2.345438 & 0.956471 & 1.158287 \\ 25 & 1 & 0 & 2.234399 & 1.424410 & 2.047542 \\ 26 & 1 & 0 & 3.237151 & 1.082957 & 0.697654 \\ 27 & 1 & 0 & -0.526970 & -2.038970 & 2.576823 \\ 28 & 6 & 0 & -2.187415 & 0.365024 & -0.299224 \\ 29 & 6 & 0 & -2.887293 & 0.043343 & 0.892415 \\ 30 & 6 & 0 & -2.572622 & -0.283888 & -1.500904 \\ 31 & 6 & 0 & -3.913910 & -0.885858 & 0.880008 \\ 32 & 1 & 0 & -2.588290 & 0.484661 & 1.836730 \\ 33 & 6 & 0 & -3.618034 & -1.191661 & -1.510122 \\ 34 & 1 & 0 & -2.086116 & -0.033153 & -2.437366 \\ 35 & 6 & 0 & -4.288065 & -1.499640 & -0.320527 \\ 36 & 1 & 0 & -4.425611 & -1.139078 & 1.803053 \\ 37 & 1 & 0 & -3.920895 & -1.658769 & -2.441767 \\ 38 & 1 & 0 & -5.102370 & -2.218259 & -0.329318\end{array}$

\section{S-TS1 in the reaction of adenine with 4-biphenylylnitrenium ion at B3PW91/6-31+G(d,p) leve1}

\section{Zero-point correction=}

Thermal correction to Energy=

Thermal correction to Enthalpy=

Thermal correction to Gibbs Free Energy=

Sum of electronic and zero-point Energies=

Sum of electronic and thermal Energies=

Sum of electronic and thermal Enthalpies=

Sum of electronic and thermal Free Energies=

\begin{tabular}{cccrrr}
$\begin{array}{c}\text { Center } \\
\text { Number }\end{array}$ & Atomic & Atomic & \multicolumn{2}{c}{ Coordinates } & (Angstroms) \\
-1 Tumber & Type & X & Y \\
1 & 7 & 0 & 3.009520 & -2.536949 & 0.857239 \\
2 & 1 & 0 & 5.186781 & -2.466750 & -0.931842 \\
3 & 7 & 0 & 3.886794 & 0.474783 & -0.863837 \\
4 & 1 & 0 & 5.554340 & -0.182310 & -2.005383 \\
5 & 6 & 0 & 3.114245 & -0.247448 & 0.012964 \\
6 & 7 & 0 & 0.488949 & 4.024417 & -0.358360 \\
7 & 6 & 0 & 3.547409 & -1.586693 & 0.103384 \\
8 & 7 & 0 & 4.616829 & -1.651654 & -0.750829 \\
9 & 6 & 0 & 1.968472 & -2.101072 & 1.570657 \\
10 & 7 & 0 & 1.454805 & -0.858220 & 1.591985 \\
11 & 6 & 0 & 2.028229 & 0.066941 & 0.828411 \\
12 & 1 & 0 & -3.418580 & 2.089866 & 0.939548 \\
13 & 6 & 0 & 4.768984 & -0.398819 & -1.292817
\end{tabular}

0. 302369 (Hartree/Particle)

0.320200

0.321144

0.253278

$-984.458303$

$-984.440472$

$-984.439528$

-984. 507394 


$\begin{array}{rrrrrr}14 & 1 & 0 & 0.102795 & 4.903908 & -0.002882 \\ 15 & 6 & 0 & -0.326405 & 3.045196 & -0.188628 \\ 16 & 6 & 0 & -2.057046 & 0.758790 & -0.157167 \\ 17 & 6 & 0 & -1.636155 & 3.104180 & 0.457837 \\ 18 & 6 & 0 & 0.125873 & 1.710086 & -0.667357 \\ 19 & 6 & 0 & -0.797924 & 0.642567 & -0.719919 \\ 20 & 6 & 0 & -2.435490 & 2.016405 & 0.487142 \\ 21 & 1 & 0 & -1.957125 & 4.049220 & 0.888493 \\ 22 & 1 & 0 & 0.950167 & 1.734466 & -1.374756 \\ 23 & 1 & 0 & -0.494139 & -0.286992 & -1.187917 \\ 24 & 7 & 0 & 1.457975 & 1.351266 & 0.845690 \\ 25 & 1 & 0 & 0.928215 & 1.504983 & 1.701705 \\ 26 & 1 & 0 & 2.112507 & 2.108884 & 0.650304 \\ 27 & 1 & 0 & 1.479854 & -2.828524 & 2.211864 \\ 28 & 6 & 0 & -3.038932 & -0.321309 & -0.236402 \\ 29 & 6 & 0 & -4.022696 & -0.481670 & 0.762146 \\ 30 & 6 & 0 & -3.016641 & -1.234758 & -1.311812 \\ 31 & 6 & 0 & -4.931246 & -1.531182 & 0.698354 \\ 32 & 1 & 0 & -4.052165 & 0.185778 & 1.617716 \\ 33 & 6 & 0 & -3.940480 & -2.267819 & -1.382670 \\ 34 & 1 & 0 & -2.303840 & -1.104042 & -2.120112 \\ 35 & 6 & 0 & -4.897037 & -2.423230 & -0.375136 \\ 36 & 1 & 0 & -5.667897 & -1.654076 & 1.486163 \\ 37 & 1 & 0 & -3.925739 & -2.947775 & -2.228825 \\ 38 & 1 & 0 & -5.618222 & -3.233131 & -0.431212\end{array}$

\section{S-INT1 in the reaction of adenine with 4-biphenylylnitrenium ion at B3PW91/6-31+G(d,p) leve1}

Zero-point correction=

Thermal correction to Energy=

Thermal correction to Enthalpy=

Thermal correction to Gibbs Free Energy=

Sum of electronic and zero-point Energies=

Sum of electronic and thermal Energies=

Sum of electronic and thermal Enthalpies=

Sum of electronic and thermal Free Energies=
0. 303919 (Hartree/Particle)

0.321373

0.322317

0.256144

$-984.477197$

$-984.459743$

$-984.458799$

$-984.524972$

\begin{tabular}{|c|c|c|c|c|c|}
\hline \multirow{2}{*}{$\begin{array}{l}\text { Center } \\
\text { Number }\end{array}$} & \multirow{2}{*}{$\begin{array}{l}\text { Atomic } \\
\text { Number }\end{array}$} & \multirow{2}{*}{$\begin{array}{l}\text { Atomic } \\
\text { Type }\end{array}$} & \multicolumn{3}{|c|}{ Coordinates (Angstroms) } \\
\hline & & & $X$ & $\mathrm{Y}$ & Z \\
\hline 1 & 7 & 0 & -4.343377 & -1.947397 & -0.745272 \\
\hline 2 & 1 & 0 & -5.370457 & -1.631331 & 1.852121 \\
\hline 3 & 7 & 0 & -2.965501 & 0.475376 & 1. 499934 \\
\hline 4 & 1 & 0 & -4.177543 & 0.163484 & 3. 215934 \\
\hline 5 & 6 & 0 & -3.017226 & -0.167267 & 0.285389 \\
\hline 6 & 7 & 0 & -0.768989 & 3. 297064 & -0.638016 \\
\hline 7 & 6 & 0 & -4.034688 & -1.151067 & 0.267578 \\
\hline 8 & 7 & 0 & -4.599282 & -1.072187 & 1.511963 \\
\hline 9 & 6 & 0 & -3.577312 & -1.750536 & -1.817583 \\
\hline 10 & 7 & 0 & -2.579300 & -0.853142 & -1.940188 \\
\hline 11 & 6 & 0 & -2.313846 & -0.075686 & -0.907518 \\
\hline 12 & 1 & 0 & 3. 406710 & 2. 275749 & 0.824887 \\
\hline 13 & 6 & 0 & -3.923089 & -0.093590 & 2. 195756 \\
\hline
\end{tabular}




$\begin{array}{rrrrrr}14 & 1 & 0 & -0.587018 & 4.298922 & -0.637481 \\ 15 & 6 & 0 & 0.149093 & 2.563190 & -0.128570 \\ 16 & 6 & 0 & 2.216253 & 0.573451 & 0.124039 \\ 17 & 6 & 0 & 1.453446 & 2.930784 & 0.386407 \\ 18 & 6 & 0 & -0.189158 & 1.076692 & -0.083438 \\ 19 & 6 & 0 & 0.973914 & 0.150251 & -0.207171 \\ 20 & 6 & 0 & 2.416626 & 1.985854 & 0.484250 \\ 21 & 1 & 0 & 1.662739 & 3.975453 & 0.597292 \\ 22 & 1 & 0 & -0.698998 & 0.911118 & 0.880459 \\ 23 & 1 & 0 & 0.783327 & -0.887764 & -0.467686 \\ 24 & 7 & 0 & -1.258588 & 0.901450 & -1.135571 \\ 25 & 1 & 0 & -0.842041 & 0.671235 & -2.045444 \\ 26 & 1 & 0 & -1.602910 & 1.925372 & -1.196986 \\ 27 & 1 & 0 & -3.771047 & -2.372430 & -2.685651 \\ 28 & 6 & 0 & 3.380435 & -0.338283 & 0.149439 \\ 29 & 6 & 0 & 4.632812 & 0.094282 & -0.315059 \\ 30 & 6 & 0 & 3.251889 & -1.652879 & 0.624028 \\ 31 & 6 & 0 & 5.722069 & -0.773221 & -0.323077 \\ 32 & 1 & 0 & 4.753897 & 1.102482 & -0.703058 \\ 33 & 6 & 0 & 4.344200 & -2.516154 & 0.621723 \\ 34 & 1 & 0 & 2.301687 & -1.989930 & 1.029716 \\ 35 & 6 & 0 & 5.581064 & -2.079621 & 0.145842 \\ 36 & 1 & 0 & 6.681237 & -0.428989 & -0.698066 \\ 37 & 1 & 0 & 4.233311 & -3.526444 & 1.004243 \\ 38 & 1 & 0 & 6.433490 & -2.752274 & 0.147566\end{array}$

TS2 in the reaction of adenine with 4-biphenylylnitrenium ion at B3PW91/6-31+G(d,p) leve1

Zero-point correction=

Thermal correction to Energy=

Thermal correction to Enthalpy=

Thermal correction to Gibbs Free Energy=

Sum of electronic and zero-point Energies=

Sum of electronic and thermal Energies=

Sum of electronic and thermal Enthalpies=

Sum of electronic and thermal Free Energies=

$\begin{array}{ccc}\text { Center } & \text { Atomic } & \text { Atomic } \\ \text { Number } & \text { Number } & \text { Type }\end{array}$

0. 300940 (Hartree/Particle)

0.317986

0. 318931

0. 253919

$-984.479673$

$-984.462626$

$-984.461682$

$-984.526693$

$\begin{array}{rrrrrr}-1 & 7 & 0 & -4.540092 & -1.780406 & -0.741258 \\ 2 & 1 & 0 & -5.379562 & -1.560006 & 1.930689 \\ 3 & 7 & 0 & -2.827601 & 0.359897 & 1.560129 \\ 4 & 1 & 0 & -3.958964 & 0.031985 & 3.327784 \\ 5 & 6 & 0 & -3.005056 & -0.192041 & 0.311306 \\ 6 & 7 & 0 & -0.832612 & 3.139997 & -0.629164 \\ 7 & 6 & 0 & -4.102183 & -1.083028 & 0.297572 \\ 8 & 7 & 0 & -4.584537 & -1.043546 & 1.578672 \\ 9 & 6 & 0 & -3.822218 & -1.573178 & -1.844358 \\ 10 & 7 & 0 & -2.757488 & -0.758058 & -1.973969 \\ 11 & 6 & 0 & -2.360633 & -0.074676 & -0.914823 \\ 12 & 1 & 0 & 3.350027 & 2.256279 & 0.898682 \\ 13 & 6 & 0 & -3.787865 & -0.174182 & 2.279154\end{array}$




$\begin{array}{rrrrrr}14 & 1 & 0 & -0.761085 & 4.153593 & -0.635610 \\ 15 & 6 & 0 & 0.114411 & 2.453588 & -0.099464 \\ 16 & 6 & 0 & 2.240465 & 0.541251 & 0.110119 \\ 17 & 6 & 0 & 1.382005 & 2.861373 & 0.454340 \\ 18 & 6 & 0 & -0.177351 & 0.958623 & -0.114297 \\ 19 & 6 & 0 & 1.021210 & 0.083253 & -0.261884 \\ 20 & 6 & 0 & 2.376574 & 1.942985 & 0.531184 \\ 21 & 1 & 0 & 1.552643 & 3.904414 & 0.702626 \\ 22 & 1 & 0 & -0.661901 & 0.747217 & 0.855658 \\ 23 & 1 & 0 & 0.875065 & -0.949740 & -0.566407 \\ 24 & 7 & 0 & -1.253724 & 0.823450 & -1.149605 \\ 25 & 1 & 0 & -0.869162 & 0.587744 & -2.070139 \\ 26 & 1 & 0 & -1.514315 & 1.968595 & -1.160385 \\ 27 & 1 & 0 & -4.119750 & -2.115595 & -2.736223 \\ 28 & 6 & 0 & 3.440901 & -0.323455 & 0.121173 \\ 29 & 6 & 0 & 4.680253 & 0.173023 & -0.312995 \\ 30 & 6 & 0 & 3.358503 & -1.656766 & 0.551083 \\ 31 & 6 & 0 & 5.803192 & -0.650290 & -0.334086 \\ 32 & 1 & 0 & 4.765934 & 1.197047 & -0.667937 \\ 33 & 6 & 0 & 4.484201 & -2.476083 & 0.535182 \\ 34 & 1 & 0 & 2.417266 & -2.044351 & 0.931857 \\ 35 & 6 & 0 & 5.708373 & -1.975937 & 0.090347 \\ 36 & 1 & 0 & 6.752300 & -0.256723 & -0.685384 \\ 37 & 1 & 0 & 4.408568 & -3.502145 & 0.882651 \\ 38 & 1 & 0 & 6.586624 & -2.614417 & 0.081174\end{array}$

INT2 in the reaction of adenine with 4-biphenylylnitrenium ion at B3PW91/6-31+G(d,p) 1eve1

Zero-point correction=

Thermal correction to Energy=

Thermal correction to Enthalpy=

Thermal correction to Gibbs Free Energy=

Sum of electronic and zero-point Energies=

Sum of electronic and thermal Energies=

Sum of electronic and thermal Enthalpies=

Sum of electronic and thermal Free Energies=
0. 303940 (Hartree/Particle)
0.321622
0.322566
0. 256239
$-984.507570$
$-984.489888$
$-984.488944$
$-984.555271$

\begin{tabular}{|c|c|c|c|c|c|}
\hline \multirow{2}{*}{$\begin{array}{l}\text { Center } \\
\text { Number }\end{array}$} & \multirow{2}{*}{$\begin{array}{l}\text { Atomic } \\
\text { Number }\end{array}$} & \multirow{2}{*}{$\begin{array}{l}\text { Atomic } \\
\text { Type }\end{array}$} & \multicolumn{3}{|c|}{ Coordinates (Angstroms) } \\
\hline & & & $X$ & $\mathrm{Y}$ & Z \\
\hline 1 & 7 & 0 & -4.635353 & -1.565452 & -0.770984 \\
\hline 2 & 1 & 0 & -4.874378 & -2.067720 & 1.983071 \\
\hline 3 & 7 & 0 & -2.319764 & -0.173381 & 1. 575690 \\
\hline 4 & 1 & 0 & -3.079031 & -0.946867 & 3. 406508 \\
\hline 5 & 6 & 0 & -2.794104 & -0.364085 & 0.291380 \\
\hline 6 & 7 & 0 & -0.918392 & 3. 389993 & 0.007472 \\
\hline 7 & 6 & 0 & -3.929669 & -1.186325 & 0.290758 \\
\hline 8 & 7 & 0 & -4.131394 & -1.489571 & 1. 615204 \\
\hline 9 & 6 & 0 & -4.135971 & -1.069670 & -1.902996 \\
\hline 10 & 7 & 0 & -3.059368 & -0.277707 & -2.053041 \\
\hline 11 & 6 & 0 & -2.379433 & 0.089632 & -0.968828 \\
\hline
\end{tabular}




$\begin{array}{rrrrrr}12 & 1 & 0 & 3.291110 & 2.167724 & 1.050675 \\ 13 & 6 & 0 & -3.145639 & -0.863051 & 2.329987 \\ 14 & 1 & 0 & -0.820922 & 4.350906 & 0.306501 \\ 15 & 6 & 0 & 0.044350 & 2.506809 & 0.161525 \\ 16 & 6 & 0 & 2.177119 & 0.569461 & 0.084069 \\ 17 & 6 & 0 & 1.315613 & 2.825903 & 0.699072 \\ 18 & 6 & 0 & -0.252387 & 1.080116 & -0.181117 \\ 19 & 6 & 0 & 0.944515 & 0.211554 & -0.367044 \\ 20 & 6 & 0 & 2.317634 & 1.892929 & 0.651935 \\ 21 & 1 & 0 & 1.508744 & 3.824700 & 1.079334 \\ 22 & 1 & 0 & -0.683029 & 0.719732 & 0.796173 \\ 23 & 1 & 0 & 0.766042 & -0.763596 & -0.812144 \\ 24 & 7 & 0 & -1.310149 & 0.952180 & -1.161197 \\ 25 & 1 & 0 & -1.034894 & 1.009619 & -2.137218 \\ 26 & 1 & 0 & -1.775019 & 3.099999 & -0.454413 \\ 27 & 1 & 0 & -4.654648 & -1.336242 & -2.819491 \\ 28 & 6 & 0 & 3.352066 & -0.328068 & 0.008449 \\ 29 & 6 & 0 & 4.610227 & 0.174157 & -0.356980 \\ 30 & 6 & 0 & 3.221043 & -1.695928 & 0.289844 \\ 31 & 6 & 0 & 5.709478 & -0.676042 & -0.449060 \\ 32 & 1 & 0 & 4.730346 & 1.226361 & -0.603989 \\ 33 & 6 & 0 & 4.322481 & -2.543278 & 0.199383 \\ 34 & 1 & 0 & 2.261742 & -2.093684 & 0.610728 \\ 35 & 6 & 0 & 5.568111 & -2.035910 & -0.171057 \\ 36 & 1 & 0 & 6.675152 & -0.277185 & -0.744667 \\ 37 & 1 & 0 & 4.210302 & -3.598441 & 0.429895 \\ 38 & 1 & 0 & 6.426824 & -2.696956 & -0.238879\end{array}$

TS3 in the reaction of adenine with 4-biphenylylnitrenium ion at B3PW91/6-31+G(d,p) 1eve1

Zero-point correction=

Thermal correction to Energy=

Thermal correction to Enthalpy=

Thermal correction to Gibbs Free Energy=

Sum of electronic and zero-point Energies=

Sum of electronic and thermal Energies=

Sum of electronic and thermal Enthalpies=

Sum of electronic and thermal Free Energies=
0.299196 (Hartree/Particle)
0.316559
0.317503
0.253012

$-984.504153$

$-984.486789$

$-984.485845$

$-984.550336$

\begin{tabular}{|c|c|c|c|c|c|}
\hline \multirow{2}{*}{$\begin{array}{l}\text { Center } \\
\text { Number }\end{array}$} & \multirow{2}{*}{$\begin{array}{l}\text { Atomic } \\
\text { Number }\end{array}$} & \multirow{2}{*}{$\begin{array}{l}\text { Atomic } \\
\text { Type }\end{array}$} & \multicolumn{3}{|c|}{ Coordinates (Angstroms) } \\
\hline & & & $X$ & $\mathrm{Y}$ & Z \\
\hline 1 & 7 & 0 & -4.611954 & -1.588295 & -0.386250 \\
\hline 2 & 1 & 0 & -4.085784 & -2.226669 & 2. 350601 \\
\hline 3 & 7 & 0 & -1.770551 & -0.299863 & 1. 313243 \\
\hline 4 & 1 & 0 & -1.965075 & -1.153430 & 3. 266566 \\
\hline 5 & 6 & 0 & -2.582847 & -0.438573 & 0.214926 \\
\hline 6 & 7 & 0 & -1.217936 & 3. 269987 & 0.153487 \\
\hline 7 & 6 & 0 & -3.662131 & -1.261522 & 0.486959 \\
\hline 8 & 7 & 0 & -3.479702 & -1.622541 & 1.810243 \\
\hline 9 & 6 & 0 & -4.398441 & -1.026661 & -1.581506 \\
\hline
\end{tabular}




$\begin{array}{rrrrrr}10 & 7 & 0 & -3.391131 & -0.215915 & -1.969618 \\ 11 & 6 & 0 & -2.461391 & 0.095496 & -1.069886 \\ 12 & 1 & 0 & 2.985836 & 2.081325 & 1.332351 \\ 13 & 6 & 0 & -2.337879 & -1.027852 & 2.259972 \\ 14 & 1 & 0 & -1.175072 & 4.158301 & 0.628995 \\ 15 & 6 & 0 & -0.195568 & 2.410345 & 0.190119 \\ 16 & 6 & 0 & 2.050923 & 0.616772 & 0.036819 \\ 17 & 6 & 0 & 1.009497 & 2.693740 & 0.878084 \\ 18 & 6 & 0 & -0.328608 & 1.096833 & -0.412678 \\ 19 & 6 & 0 & 0.873129 & 0.306280 & -0.599447 \\ 20 & 6 & 0 & 2.074530 & 1.827933 & 0.797200 \\ 21 & 1 & 0 & 1.104695 & 3.625893 & 1.427591 \\ 22 & 1 & 0 & -0.774682 & 0.480726 & 0.707188 \\ 23 & 1 & 0 & 0.787977 & -0.587063 & -1.212540 \\ 24 & 7 & 0 & -1.417364 & 0.932938 & -1.355164 \\ 25 & 1 & 0 & -1.257636 & 1.139100 & -2.335528 \\ 26 & 1 & 0 & -2.016643 & 3.078143 & -0.434893 \\ 27 & 1 & 0 & -5.132600 & -1.251848 & -2.349772 \\ 28 & 6 & 0 & 3.255803 & -0.237588 & -0.067776 \\ 29 & 6 & 0 & 4.526715 & 0.338336 & -0.215198 \\ 30 & 6 & 0 & 3.147441 & -1.635633 & -0.030443 \\ 31 & 6 & 0 & 5.659013 & -0.465080 & -0.327408 \\ 32 & 1 & 0 & 4.632476 & 1.418716 & -0.274681 \\ 33 & 6 & 0 & 4.280773 & -2.437482 & -0.143071 \\ 34 & 1 & 0 & 2.175076 & -2.099492 & 0.115488 \\ 35 & 6 & 0 & 5.539402 & -1.854498 & -0.291581 \\ 36 & 1 & 0 & 6.634693 & -0.004882 & -0.451939 \\ 37 & 1 & 0 & 4.182098 & -3.518302 & -0.104066 \\ 38 & 1 & 0 & 6.422949 & -2.479787 & -0.377456\end{array}$

INT3 in the reaction of adenine with 4-biphenylylnitrenium ion at B3PW91/6-31+G(d,p) leve1

Zero-point correction=

Thermal correction to Energy=

Thermal correction to Enthalpy=

Thermal correction to Gibbs Free Energy=

Sum of electronic and zero-point Energies=

Sum of electronic and thermal Energies=

Sum of electronic and thermal Enthalpies=

Sum of electronic and thermal Free Energies=

$\begin{array}{ccc}\text { Center } & \text { Atomic } & \text { Atomic } \\ \text { Number } & \text { Number } & \text { Type }\end{array}$

\author{
0. 305177 (Hartree/Particle) \\ 0. 322947 \\ 0.323891 \\ 0. 257768 \\ $-984.538567$ \\ $-984.520797$ \\ $-984.519853$ \\ $-984.585976$
}

$\begin{array}{rrrrrr}1 & 7 & 0 & -4.661332 & -1.527641 & -0.202917 \\ 2 & 1 & 0 & -4.505546 & -1.465429 & 2.567401 \\ 3 & 7 & 0 & -1.969475 & 0.027059 & 1.447708 \\ 4 & 1 & 0 & -2.455011 & -0.261357 & 3.514455 \\ 5 & 6 & 0 & -2.595742 & -0.329630 & 0.262615 \\ 6 & 7 & 0 & -1.471388 & 2.871207 & -0.220796 \\ 7 & 6 & 0 & -3.760325 & -1.010840 & 0.615614\end{array}$




\begin{tabular}{|c|c|c|c|c|c|}
\hline 8 & 7 & 0 & -3.780635 & -1.035854 & 2. 004574 \\
\hline 9 & 6 & 0 & -4.332372 & -1.317305 & -1.483637 \\
\hline 10 & 7 & 0 & -3.256000 & -0.688620 & -1.963117 \\
\hline 11 & 6 & 0 & -2.344003 & -0.177145 & -1.124574 \\
\hline 12 & 1 & 0 & 2. 793956 & 2. 154851 & 1. 273631 \\
\hline 13 & 6 & 0 & -2.697934 & -0.402583 & 2. 471328 \\
\hline 14 & 1 & 0 & -1.392216 & 3. 840180 & 0.057054 \\
\hline 15 & 6 & 0 & -0.283649 & 2. 150292 & -0.187726 \\
\hline 16 & 6 & 0 & 2. 117324 & 0.600861 & -0.060762 \\
\hline 17 & 6 & 0 & 0.809178 & 2. 559069 & 0.596652 \\
\hline 18 & 6 & 0 & -0.163700 & 0.919491 & -0.866835 \\
\hline 19 & 6 & 0 & 1. 016961 & 0.175648 & -0.813877 \\
\hline 20 & 6 & 0 & 1. 974738 & 1. 804544 & 0.652460 \\
\hline 21 & 1 & 0 & 0.745795 & 3. 492645 & 1. 150498 \\
\hline 22 & 1 & 0 & -1.111891 & 0.569458 & 1. 514637 \\
\hline 23 & 1 & 0 & 1. 074735 & -0.742948 & -1.391772 \\
\hline 24 & 7 & 0 & -1.266033 & 0.444190 & -1.644795 \\
\hline 25 & 1 & 0 & -1.187598 & 0.381983 & -2.654689 \\
\hline 26 & 1 & 0 & -2.016388 & 2. 766456 & -1.067263 \\
\hline 27 & 1 & 0 & -5.022496 & -1.707003 & -2.226695 \\
\hline 28 & 6 & 0 & 3. 371541 & -0.185219 & -0.015488 \\
\hline 29 & 6 & 0 & 4. 618102 & 0.459493 & -0.017391 \\
\hline 30 & 6 & 0 & 3. 343151 & -1.587817 & 0.024556 \\
\hline 31 & 6 & 0 & 5.799812 & -0.277262 & 0.019805 \\
\hline 32 & 1 & 0 & 4. 665955 & 1. 543452 & -0.079804 \\
\hline 33 & 6 & 0 & 4. 525152 & -2.324020 & 0.060532 \\
\hline 34 & 1 & 0 & 2. 389493 & -2.109355 & 0.052945 \\
\hline 35 & 6 & 0 & 5. 757914 & -1.671053 & 0.058897 \\
\hline 36 & 1 & 0 & 6.755377 & 0.238848 & 0.006875 \\
\hline 37 & 1 & 0 & 4. 483388 & -3.408781 & 0.098485 \\
\hline 38 & 1 & 0 & 6. 679603 & -2.244556 & 0.086626 \\
\hline
\end{tabular}

TS1 in the reaction of adenine with 4-biphenylylnitrenium ion at B3PW91/6-31+G(d,p) leve1

Zero-point correction=

Thermal correction to Energy=

Thermal correction to Enthalpy=

Thermal correction to Gibbs Free Energy=

Sum of electronic and zero-point Energies=

Sum of electronic and thermal Energies=

Sum of electronic and thermal Enthalpies=

Sum of electronic and thermal Free Energies=

$\begin{array}{ccc}\text { Center } & \text { Atomic } & \text { Atomic } \\ \text { Number } & \text { Number } & \text { Type }\end{array}$

0. 302485 (Hartree/Particle)

0. 320233

0. 321177

0. 254280

$-984.458847$

$-984.441099$

$-984.440155$

$-984.507052$

$\begin{array}{rrrrrr}1 & 7 & 0 & 5.006526 & 1.190220 & -0.085291 \\ 2 & 1 & 0 & 6.066936 & -1.408226 & -0.312209 \\ 3 & 7 & 0 & 2.899107 & -1.599665 & -0.842499 \\ 4 & 1 & 0 & 4.319945 & -3.179787 & -0.868428 \\ 5 & 6 & 0 & 3.111942 & -0.263641 & -0.597871 \\ 6 & 7 & 0 & 1.371139 & -0.377951 & 2.171869 \\ 7 & 6 & 0 & 4.474411 & 0.004669 & -0.340001\end{array}$




\begin{tabular}{rrrrrr}
8 & 7 & 0 & 5.080466 & -1.221292 & -0.430877 \\
9 & 6 & 0 & 4.104603 & 2.176698 & -0.101845 \\
10 & 7 & 0 & 2.788572 & 2.071936 & -0.347510 \\
11 & 6 & 0 & 2.295796 & 0.864690 & -0.599339 \\
12 & 1 & 0 & -2.438000 & -2.322719 & 0.636454 \\
13 & 6 & 0 & 4.096554 & -2.129229 & -0.734290 \\
14 & 1 & 0 & 1.605528 & -1.301153 & 2.549894 \\
15 & 6 & 0 & 0.280438 & -0.421194 & 1.492300 \\
16 & 6 & 0 & -2.344612 & -0.142891 & 0.387697 \\
17 & 6 & 0 & -0.563610 & -1.592299 & 1.268958 \\
18 & 6 & 0 & -0.173495 & 0.850733 & 0.877129 \\
19 & 6 & 0 & -1.527421 & 0.970146 & 0.469725 \\
20 & 6 & 0 & -1.798662 & -1.452328 & 0.738551 \\
21 & 1 & 0 & -0.196348 & -2.563799 & 1.588665 \\
22 & 1 & 0 & 0.367202 & 1.732906 & 1.210532 \\
23 & 1 & 0 & -1.904972 & 1.947534 & 0.186847 \\
24 & 7 & 0 & 0.906425 & 0.785255 & -0.822488 \\
25 & 1 & 0 & 0.554748 & 1.620008 & -1.287544 \\
26 & 1 & 0 & 0.619890 & -0.066239 & -1.303036 \\
27 & 1 & 0 & 4.474712 & 3.177287 & 0.099537 \\
28 & 6 & 0 & -3.751163 & -0.038214 & -0.006285 \\
29 & 6 & 0 & -4.393705 & -1.095027 & -0.683046 \\
30 & 6 & 0 & -4.489506 & 1.129890 & 0.274699 \\
31 & 6 & 0 & -5.718269 & -0.974849 & -1.087043 \\
32 & 1 & 0 & -3.847935 & -1.999129 & -0.935241 \\
33 & 6 & 0 & -5.818733 & 1.236667 & -0.111932 \\
34 & 1 & 0 & -4.033233 & 1.936704 & 0.840234 \\
35 & 6 & 0 & -6.435558 & 0.187693 & -0.799280 \\
36 & 1 & 0 & -6.192840 & -1.788935 & -1.626066 \\
37 & 1 & 0 & -6.380659 & 2.133056 & 0.131646 \\
38 & 1 & 0 & -7.474686 & 0.273883 & -1.102486 \\
& & & & & \\
\hline 3 & & 0 & & &
\end{tabular}

\section{INT1 in the reaction of adenine with 4-biphenylylnitrenium ion at B3PW91/6-31+G(d,p) level}

Zero-point correction=

Thermal correction to Energy=

Thermal correction to Enthalpy=

Thermal correction to Gibbs Free Energy=

Sum of electronic and zero-point Energies=

Sum of electronic and thermal Energies=

Sum of electronic and thermal Enthalpies=

Sum of electronic and thermal Free Energies=

$\begin{array}{ccc}\text { Center } & \text { Atomic } & \text { Atomic } \\ \text { Number } & \text { Number } & \text { Type }\end{array}$

0. 304912 (Hartree/Particle)

0. 322723

0. 323667

0. 256463

$-984.463692$

$-984.445881$

$-984.444937$

$-984.512141$

Coordinates (Angstroms)

$\begin{array}{lll}X & Y & Z\end{array}$

$\begin{array}{lrr}4.943080 & 1.351574 & 0.010519 \\ 6.265290 & -1.064498 & -0.611912 \\ 3.109751 & -1.536066 & -1.038069 \\ 4.685639 & -2.929826 & -1.333462 \\ 3.196132 & -0.230496 & -0.625348 \\ 1.361181 & -0.610298 & 2.149557 \\ 4.535797 & 0.157044 & -0.388679 \\ 5.259865 & -0.970486 & -0.669916\end{array}$




\begin{tabular}{rrrrrr}
9 & 6 & 0 & 3.944919 & 2.221777 & \multicolumn{1}{c}{0.176213} \\
10 & 7 & 0 & 2.631900 & 1.989182 & -0.017669 \\
11 & 6 & 0 & 2.282731 & 0.780783 & -0.399676 \\
12 & 1 & 0 & -2.691995 & -2.287489 & 0.955075 \\
13 & 6 & 0 & 4.360730 & -1.936712 & -1.050998 \\
14 & 1 & 0 & 1.501673 & -1.491376 & 2.651375 \\
15 & 6 & 0 & 0.249800 & -0.611812 & 1.513782 \\
16 & 6 & 0 & -2.371445 & -0.197581 & 0.355454 \\
17 & 6 & 0 & -0.741548 & -1.682574 & 1.476197 \\
18 & 6 & 0 & -0.048981 & 0.632944 & 0.687726 \\
19 & 6 & 0 & -1.460346 & 0.799782 & 0.243997 \\
20 & 6 & 0 & -1.958209 & -1.487703 & 0.927672 \\
21 & 1 & 0 & -0.483166 & -2.629912 & 1.942441 \\
22 & 1 & 0 & 0.343527 & 1.515705 & 1.199972 \\
23 & 1 & 0 & -1.742946 & 1.762607 & -0.175941 \\
24 & 7 & 0 & 0.843823 & 0.560987 & -0.603098 \\
25 & 1 & 0 & 0.496299 & 1.286240 & -1.239677 \\
26 & 1 & 0 & 0.699247 & -0.346517 & -1.062203 \\
27 & 1 & 0 & 4.212737 & 3.223515 & 0.496048 \\
28 & 6 & 0 & -3.781684 & -0.019601 & -0.046266 \\
29 & 6 & 0 & -4.484068 & -1.062469 & -0.672737 \\
30 & 6 & 0 & -4.440528 & 1.200061 & 0.180505 \\
31 & 6 & 0 & -5.801840 & -0.880213 & -1.083398 \\
32 & 1 & 0 & -3.994188 & -2.011840 & -0.872390 \\
33 & 6 & 0 & -5.761304 & 1.375394 & -0.221243 \\
34 & 1 & 0 & -3.928504 & 2.000038 & 0.708298 \\
35 & 6 & 0 & -6.444145 & 0.337568 & -0.857459 \\
36 & 1 & 0 & -6.327760 & -1.690329 & -1.579558 \\
37 & 1 & 0 & -6.263208 & 2.318045 & -0.024669 \\
38 & 1 & 0 & -7.475908 & 0.474211 & -1.167123
\end{tabular}

COM21 in the reaction of adenine with 4-biphenylylnitrenium ion at B3LYP/6-31+G(d,p) leve1

Zero-point correction=

Thermal correction to Energy=

Thermal correction to Enthalpy=

Thermal correction to Gibbs Free Energy=

Sum of electronic and zero-point Energies=

Sum of electronic and thermal Energies=

Sum of electronic and thermal Enthalpies=

Sum of electronic and thermal Free Energies=
0. 299790 (Hartree/Particle)

0.318278

0. 319222

0. 249576

$-984.851768$

$-984.833280$

$-984.832336$

$-984.901982$

Coordinates (Angstroms)

$\begin{array}{ccccc}\text { Center } & \text { Atomic } & \text { Atomic } & \text { Type } & \text { X }\end{array}$

\begin{tabular}{|c|c|c|c|c|c|}
\hline 1 & 7 & 0 & 3. 386331 & -0.144489 & 1.563947 \\
\hline 2 & 1 & 0 & 5. 290920 & -0.594860 & -0.470592 \\
\hline 3 & 7 & 0 & 2.517586 & -1.915424 & -1.432714 \\
\hline 4 & 1 & 0 & 4. 367516 & -1.875142 & -2.484718 \\
\hline 5 & 6 & 0 & 2. 219996 & -1.385437 & -0.191485 \\
\hline 6 & 7 & 0 & -5.516950 & -1.662365 & 0.137753 \\
\hline 7 & 6 & 0 & 3. 336711 & -0.748413 & 0.373904 \\
\hline 8 & 7 & 0 & 4. 332791 & -0.904510 & -0.563142 \\
\hline
\end{tabular}




\begin{tabular}{rrrrrr}
9 & 6 & 0 & 2.217035 & -0.212400 & \multicolumn{1}{c}{2.209579} \\
10 & 7 & 0 & 1.073433 & -0.794284 & 1.800103 \\
11 & 6 & 0 & 1.058513 & -1.403040 & 0.602349 \\
12 & 1 & 0 & -2.286326 & 0.363663 & -2.349583 \\
13 & 6 & 0 & 3.783712 & -1.611100 & -1.613255 \\
14 & 1 & 0 & -5.925722 & -1.948209 & -0.759347 \\
15 & 6 & 0 & -4.437867 & -0.966252 & -0.025034 \\
16 & 6 & 0 & -2.051762 & 0.610361 & -0.186231 \\
17 & 6 & 0 & -3.834910 & -0.627113 & -1.315074 \\
18 & 6 & 0 & -3.754444 & -0.487104 & 1.175705 \\
19 & 6 & 0 & -2.622652 & 0.261246 & 1.090937 \\
20 & 6 & 0 & -2.692750 & 0.104499 & -1.380123 \\
21 & 1 & 0 & -4.328541 & -0.966282 & -2.221921 \\
22 & 1 & 0 & -4.189318 & -0.763736 & 2.130911 \\
23 & 1 & 0 & -2.120139 & 0.571062 & 1.998286 \\
24 & 7 & 0 & -0.108270 & -1.991078 & 0.180190 \\
25 & 1 & 0 & -0.736910 & -2.268363 & 0.923745 \\
26 & 1 & 0 & -0.016424 & -2.656800 & -0.577923 \\
27 & 1 & 0 & 2.182461 & 0.254704 & 3.189790 \\
28 & 6 & 0 & -0.921323 & 1.500809 & -0.277969 \\
29 & 6 & 0 & -0.584851 & 2.361027 & 0.805828 \\
30 & 6 & 0 & -0.131088 & 1.574816 & -1.460811 \\
31 & 6 & 0 & 0.461357 & 3.263435 & 0.698293 \\
32 & 1 & 0 & -1.174765 & 2.352904 & 1.713881 \\
33 & 6 & 0 & 0.929433 & 2.461731 & -1.550526 \\
34 & 1 & 0 & -0.321241 & 0.903284 & -2.289391 \\
35 & 6 & 0 & 1.224101 & 3.315038 & -0.476637 \\
36 & 1 & 0 & 0.687902 & 3.929696 & 1.523823 \\
37 & 1 & 0 & 1.532574 & 2.493025 & -2.451740 \\
38 & 1 & 0 & 2.048298 & 4.017492 & -0.554860
\end{tabular}

TS21 in the reaction of adenine with 4-biphenylylnitrenium ion at B3LYP/6-31+G(d,p) leve1

Zero-point correction=

Thermal correction to Energy=

Thermal correction to Enthalpy=

Thermal correction to Gibbs Free Energy=

Sum of electronic and zero-point Energies=

Sum of electronic and thermal Energies=

Sum of electronic and thermal Enthalpies=

Sum of electronic and thermal Free Energies=
0. 301742（Hartree/Particle)
0. 319295
0. 320239
0. 254437
$-984.834659$
$-984.817106$
$-984.816162$
$-984.881965$

\begin{tabular}{|c|c|c|c|c|c|}
\hline \multirow{2}{*}{$\begin{array}{l}\text { Center } \\
\text { Number }\end{array}$} & \multirow{2}{*}{$\begin{array}{l}\text { Atomic } \\
\text { Number }\end{array}$} & \multirow{2}{*}{$\begin{array}{l}\text { Atomic } \\
\text { Type }\end{array}$} & \multicolumn{3}{|c|}{ Coordinates (Angstroms) } \\
\hline & & & $X$ & Y & Z \\
\hline 1 & 7 & 0 & 3. 272049 & -1.534581 & 1. 471820 \\
\hline 2 & 1 & 0 & 5. 280966 & -0.681054 & -0.329123 \\
\hline 3 & 7 & 0 & 2.442840 & -0.446765 & -1.839207 \\
\hline 4 & 1 & 0 & 4. 369507 & 0.018191 & -2.611268 \\
\hline 5 & 6 & 0 & 2. 111248 & -0.914725 & -0.585868 \\
\hline 6 & 7 & 0 & -5.462534 & -1.377143 & -0.188562 \\
\hline 7 & 6 & 0 & 3. 258947 & -1.079509 & 0.225221 \\
\hline 8 & 7 & 0 & 4. 300132 & -0.685200 & -0.579466 \\
\hline
\end{tabular}




$\begin{array}{rlrrrr}9 & 6 & 0 & 2.055312 & -1.853230 & 1.928778 \\ 10 & 7 & 0 & 0.885569 & -1.754665 & 1.262140 \\ 11 & 6 & 0 & 0.919217 & -1.289585 & 0.021367 \\ 12 & 1 & 0 & -1.980288 & 0.752162 & -2.215530 \\ 13 & 6 & 0 & 3.751814 & -0.325421 & -1.792192 \\ 14 & 1 & 0 & -5.960237 & -1.233656 & -1.073121 \\ 15 & 6 & 0 & -4.266914 & -0.903550 & -0.231078 \\ 16 & 6 & 0 & -1.523066 & 0.108659 & -0.147425 \\ 17 & 6 & 0 & -3.650359 & -0.236421 & -1.390651 \\ 18 & 6 & 0 & -3.424861 & -1.031138 & 0.967242 \\ 19 & 6 & 0 & -2.166571 & -0.558918 & 1.017657 \\ 20 & 6 & 0 & -2.386151 & 0.223567 & -1.358346 \\ 21 & 1 & 0 & -4.259677 & -0.095075 & -2.280043 \\ 22 & 1 & 0 & -3.877498 & -1.520665 & 1.823981 \\ 23 & 1 & 0 & -1.566303 & -0.687831 & 1.910845 \\ 24 & 7 & 0 & -0.335252 & -1.190687 & -0.686201 \\ 25 & 1 & 0 & -0.881727 & -2.046991 & -0.565659 \\ 26 & 1 & 0 & -0.158754 & -1.038799 & -1.682914 \\ 27 & 1 & 0 & 1.998179 & -2.238185 & 2.941427 \\ 28 & 6 & 0 & -0.681352 & 1.321597 & 0.132921 \\ 29 & 6 & 0 & -0.674708 & 1.903117 & 1.413094 \\ 30 & 6 & 0 & 0.054395 & 1.943207 & -0.895145 \\ 31 & 6 & 0 & 0.041824 & 3.076261 & 1.655682 \\ 32 & 1 & 0 & -1.251392 & 1.464808 & 2.219070 \\ 33 & 6 & 0 & 0.777239 & 3.107154 & -0.647192 \\ 34 & 1 & 0 & 0.086418 & 1.518563 & -1.894059 \\ 35 & 6 & 0 & 0.769556 & 3.680310 & 0.629086 \\ 36 & 1 & 0 & 0.023393 & 3.518947 & 2.646331 \\ 37 & 1 & 0 & 1.340456 & 3.570461 & -1.450995 \\ 38 & 1 & 0 & 1.323906 & 4.594166 & 0.818493\end{array}$

INT21 in the reaction of adenine with 4-biphenylylnitrenium ion at B3LYP/6-31+G(d,p) leve1

Zero-point correction=

Thermal correction to Energy=

Thermal correction to Enthalpy=

Thermal correction to Gibbs Free Energy=

Sum of electronic and zero-point Energies=

Sum of electronic and thermal Energies=

Sum of electronic and thermal Enthalpies=

Sum of electronic and thermal Free Energies=
0. 302805 (Hartree/Particle)

0.320709

0. 321653

0. 255134

$-984.834023$

$-984.816119$

$-984.815174$

$-984.881694$

Coordinates (Angstroms)

\begin{tabular}{|c|c|c|c|c|c|}
\hline \multirow{2}{*}{$\begin{array}{l}\text { Center } \\
\text { Number }\end{array}$} & \multirow{2}{*}{$\begin{array}{l}\text { Atomic } \\
\text { Number }\end{array}$} & \multirow{2}{*}{$\begin{array}{c}\text { Atomic } \\
\text { Type }\end{array}$} & \multicolumn{3}{|c|}{ Coordinates (Angstroms) } \\
\hline & & & X & $\mathrm{Y}$ & Z \\
\hline 1 & 7 & 0 & 3. 326234 & -1.436240 & 1. 518531 \\
\hline 2 & 1 & 0 & 5. 346502 & -0.619125 & -0.291377 \\
\hline 3 & 7 & 0 & 2.514086 & -0.415766 & -1.817424 \\
\hline 4 & 1 & 0 & 4. 444304 & 0.033122 & -2.590494 \\
\hline 5 & 6 & 0 & 2. 178198 & -0.858153 & -0.557114 \\
\hline 6 & 7 & 0 & -5.427323 & -1.444637 & -0.172294 \\
\hline 7 & 6 & 0 & 3. 322402 & -1.008038 & 0.262961 \\
\hline 8 & 7 & 0 & 4. 366562 & -0.631252 & -0.545844 \\
\hline
\end{tabular}




$\begin{array}{rlrrrr}9 & 6 & 0 & 2.107441 & -1.741404 & 1.977757 \\ 10 & 7 & 0 & 0.940677 & -1.652581 & 1.301536 \\ 11 & 6 & 0 & 0.986815 & -1.210165 & 0.058217 \\ 12 & 1 & 0 & -1.928737 & 0.585861 & -2.272648 \\ 13 & 6 & 0 & 3.823359 & -0.294604 & -1.767345 \\ 14 & 1 & 0 & -5.936618 & -1.290222 & -1.048112 \\ 15 & 6 & 0 & -4.222072 & -1.002474 & -0.237506 \\ 16 & 6 & 0 & -1.437824 & -0.036218 & -0.197812 \\ 17 & 6 & 0 & -3.607138 & -0.355043 & -1.410503 \\ 18 & 6 & 0 & -3.359639 & -1.146985 & 0.945733 \\ 19 & 6 & 0 & -2.093107 & -0.703614 & 0.979540 \\ 20 & 6 & 0 & -2.335043 & 0.075366 & -1.403235 \\ 21 & 1 & 0 & -4.225187 & -0.210313 & -2.293374 \\ 22 & 1 & 0 & -3.807608 & -1.628836 & 1.809375 \\ 23 & 1 & 0 & -1.482743 & -0.840391 & 1.864925 \\ 24 & 7 & 0 & -0.273009 & -1.133913 & -0.689674 \\ 25 & 1 & 0 & -0.745985 & -2.043600 & -0.655808 \\ 26 & 1 & 0 & -0.044093 & -0.934952 & -1.670261 \\ 27 & 1 & 0 & 2.043433 & -2.105184 & 2.997576 \\ 28 & 6 & 0 & -0.725447 & 1.274553 & 0.103033 \\ 29 & 6 & 0 & -0.880719 & 1.899803 & 1.349379 \\ 30 & 6 & 0 & 0.036589 & 1.918584 & -0.888484 \\ 31 & 6 & 0 & -0.283465 & 3.137539 & 1.599808 \\ 32 & 1 & 0 & -1.483863 & 1.439397 & 2.123561 \\ 33 & 6 & 0 & 0.639172 & 3.149289 & -0.632850 \\ 34 & 1 & 0 & 0.178686 & 1.470798 & -1.868455 \\ 35 & 6 & 0 & 0.478584 & 3.763961 & 0.612734 \\ 36 & 1 & 0 & -0.421222 & 3.611226 & 2.566521 \\ 37 & 1 & 0 & 1.224879 & 3.631285 & -1.409240 \\ 38 & 1 & 0 & 0.939024 & 4.727285 & 0.808131\end{array}$

INT22 in the reaction of adenine with 4-biphenylylnitrenium ion at B3LYP/6 $-31+G(d, p)$ leve1

Zero-point correction=

Thermal correction to Energy=

Thermal correction to Enthalpy=

Thermal correction to Gibbs Free Energy=

Sum of electronic and zero-point Energies=

Sum of electronic and thermal Energies=

Sum of electronic and thermal Enthalpies=

Sum of electronic and thermal Free Energies=

\begin{tabular}{|c|c|c|c|c|c|}
\hline \multirow{2}{*}{$\begin{array}{l}\text { Center } \\
\text { Number }\end{array}$} & \multirow{2}{*}{$\begin{array}{l}\text { Atomic } \\
\text { Number }\end{array}$} & \multirow{2}{*}{$\begin{array}{l}\text { Atomic } \\
\text { Type }\end{array}$} & \multicolumn{3}{|c|}{ Coordinates (Angstroms) } \\
\hline & & & X & $Y$ & Z \\
\hline 1 & 7 & 0 & 3. 608492 & -0.066510 & 1. 301169 \\
\hline 2 & 1 & 0 & 5. 198318 & -1.557051 & -0.504201 \\
\hline 3 & 7 & 0 & 2. 110217 & -2.048969 & -1.282287 \\
\hline 4 & 1 & 0 & 3. 833723 & -2.865419 & -2.224534 \\
\hline 5 & 6 & 0 & 2. 047470 & -1.193355 & -0.202952 \\
\hline 6 & 7 & 0 & -4.382605 & -2.859518 & 0.266082 \\
\hline 7 & 6 & 0 & 3. 335429 & -0.867333 & 0.275247 \\
\hline 8 & 7 & 0 & 4. 187635 & -1.554229 & -0.555429 \\
\hline
\end{tabular}

\begin{tabular}{|c|c|c|}
\hline $\begin{array}{r}0.301800 \\
0.319883 \\
0.320827 \\
0.254768 \\
-984 . \\
-984 . \\
-984 . \\
-984 .\end{array}$ & $\begin{array}{l}9017 \\
934 \\
9990 \\
5049 \\
\text { ordinates }\end{array}$ & Angstroms) \\
\hline X & $\mathrm{Y}$ & Z \\
\hline 3. 608492 & -0.066510 & 1. 301169 \\
\hline 5. 198318 & -1.557051 & -0.504201 \\
\hline 2. 110217 & -2.048969 & -1.282287 \\
\hline 3. 833723 & -2.865419 & -2.224534 \\
\hline 2. 047470 & -1.193355 & -0.202952 \\
\hline-4.382605 & -2.859518 & 0.266082 \\
\hline 3. 335429 & -0.867333 & 0.275247 \\
\hline 4. 187635 & -1.554229 & -0.555429 \\
\hline
\end{tabular}




\begin{tabular}{|c|c|c|c|c|c|}
\hline 9 & 6 & 0 & 2. 512344 & 0.424761 & 1. 885914 \\
\hline 10 & 7 & 0 & 1. 227872 & 0.185718 & 1. 541737 \\
\hline 11 & 6 & 0 & 1.001849 & -0.607464 & 0.506256 \\
\hline 12 & 1 & 0 & -2.324865 & 0.300943 & -2.237132 \\
\hline 13 & 6 & 0 & 3. 399948 & -2.238608 & -1.456977 \\
\hline 14 & 1 & 0 & -5.049176 & -2.977261 & -0.480778 \\
\hline 15 & 6 & 0 & -3.413389 & -1.906156 & 0.195062 \\
\hline 16 & 6 & 0 & -1.449913 & 0.211987 & -0.213513 \\
\hline 17 & 6 & 0 & -3.258085 & -1.220569 & -1.096003 \\
\hline 18 & 6 & 0 & -2.600752 & -1.558987 & 1. 244033 \\
\hline 19 & 6 & 0 & -1.565848 & -0.553783 & 1. 063369 \\
\hline 20 & 6 & 0 & -2.359719 & -0.236677 & -1.296049 \\
\hline 21 & 1 & 0 & -3.931213 & -1.498764 & -1.902837 \\
\hline 22 & 1 & 0 & -2.719452 & -2.001821 & 2. 226385 \\
\hline 23 & 1 & 0 & -1.143801 & -0.086323 & 1. 948064 \\
\hline 24 & 7 & 0 & -0.346664 & -0.893818 & 0.127782 \\
\hline 25 & 1 & 0 & -0.442457 & -1.733767 & -0.443784 \\
\hline 26 & 1 & 0 & 2. 662947 & 1. 083529 & 2. 734649 \\
\hline 27 & 6 & 0 & -0.963890 & 1. 622741 & -0.277369 \\
\hline 28 & 6 & 0 & -1.293504 & 2. 531228 & 0.736990 \\
\hline 29 & 6 & 0 & -0.232137 & 2. 061373 & -1.392818 \\
\hline 30 & 6 & 0 & -0.891966 & 3. 864634 & 0.639140 \\
\hline 31 & 1 & 0 & -1.869394 & 2. 204485 & 1. 597480 \\
\hline 32 & 6 & 0 & 0.179657 & 3. 390592 & -1.480404 \\
\hline 33 & 1 & 0 & 0.023528 & 1. 365890 & -2.188543 \\
\hline 34 & 6 & 0 & -0.151716 & 4. 293890 & -0.464914 \\
\hline 35 & 1 & 0 & -1.156112 & 4. 565277 & 1. 424732 \\
\hline 36 & 1 & 0 & 0.752430 & 3. 722817 & -2.340424 \\
\hline 37 & 1 & 0 & 0.162919 & 5. 330256 & -0.537589 \\
\hline 38 & 1 & 0 & -4.606688 & -3.301150 & 1. 145073 \\
\hline
\end{tabular}

C0M21 in the reaction of adenine with 4-biphenylylnitrenium ion at B3PW91/6-31+G(d,p) leve1

Zero-point correction=

Thermal correction to Energy=

Thermal correction to Enthalpy=

Thermal correction to Gibbs Free Energy=

Sum of electronic and zero-point Energies=

Sum of electronic and thermal Energies=

Sum of electronic and thermal Enthalpies=

Sum of electronic and thermal Free Energies=
0. 300756 (Hartree/Particle)
0. 319293
0. 320237
0. 250208
$-984.463179$
$-984.444642$
$-984.443698$
$-984.513727$

\begin{tabular}{cccrrr}
$\begin{array}{c}\text { Center } \\
\text { Number }\end{array}$ & $\begin{array}{c}\text { Atomic } \\
\text { Number }\end{array}$ & $\begin{array}{c}\text { Atomic } \\
\text { Type }\end{array}$ & \multicolumn{3}{c}{ Coordinates (Angstroms) } \\
------1 & $X$ & $Y$ & $Z$ \\
1 & 7 & 0 & 3.386433 & -0.175310 & 1.576897 \\
2 & 1 & 0 & 5.302869 & -0.587385 & -0.442762 \\
3 & 7 & 0 & 2.539356 & -1.873120 & -1.459746 \\
4 & 1 & 0 & 4.399259 & -1.818098 & -2.488895 \\
5 & 6 & 0 & 2.232994 & -1.369779 & -0.215396 \\
6 & 7 & 0 & -5.494619 & -1.689095 & 0.134772 \\
7 & 6 & 0 & 3.344151 & -0.751555 & 0.376429 \\
8 & 7 & 0 & 4.345859 & -0.892239 & -0.550816
\end{tabular}




\begin{tabular}{|c|c|c|c|c|c|}
\hline 9 & 6 & 0 & 2. 211735 & -0.252421 & 2. 205613 \\
\hline 10 & 7 & 0 & 1. 072154 & -0.818216 & 1. 775303 \\
\hline 11 & 6 & 0 & 1. 065828 & -1.399029 & 0.566845 \\
\hline 12 & 1 & 0 & -2.306019 & 0.407912 & -2.340879 \\
\hline 13 & 6 & 0 & 3. 806868 & -1.570278 & -1.617807 \\
\hline 14 & 1 & 0 & -5.913376 & -1.950328 & -0.764065 \\
\hline 15 & 6 & 0 & -4.420249 & -0.988520 & -0.025658 \\
\hline 16 & 6 & 0 & -2.048971 & 0.595761 & -0.177133 \\
\hline 17 & 6 & 0 & -3.837360 & -0.616775 & -1.312113 \\
\hline 18 & 6 & 0 & -3.723841 & -0.541459 & 1. 175969 \\
\hline 19 & 6 & 0 & -2.597288 & 0.212617 & 1. 096204 \\
\hline 20 & 6 & 0 & -2.699272 & 0.118890 & -1.373378 \\
\hline 21 & 1 & 0 & -4.343061 & -0.933057 & -2.221212 \\
\hline 22 & 1 & 0 & -4.145585 & -0.846754 & 2. 128992 \\
\hline 23 & 1 & 0 & -2.079003 & 0.499620 & 2. 003394 \\
\hline 24 & 7 & 0 & -0.093883 & -1.973662 & 0.124805 \\
\hline 25 & 1 & 0 & -0.731907 & -2.260953 & 0.854680 \\
\hline 26 & 1 & 0 & -0.000136 & -2.617903 & -0.649850 \\
\hline 27 & 1 & 0 & 2. 169490 & 0. 193469 & 3. 196166 \\
\hline 28 & 6 & 0 & -0.932144 & 1. 500093 & -0.261686 \\
\hline 29 & 6 & 0 & -0.611785 & 2. 353903 & 0.827400 \\
\hline 30 & 6 & 0 & -0.147655 & 1. 595079 & -1.442487 \\
\hline 31 & 6 & 0 & 0.415798 & 3. 275189 & 0.725616 \\
\hline 32 & 1 & 0 & -1.204410 & 2. 328850 & 1. 734533 \\
\hline 33 & 6 & 0 & 0.896050 & 2. 499156 & -1.525803 \\
\hline 34 & 1 & 0 & -0.327890 & 0.924413 & -2.275222 \\
\hline 35 & 6 & 0 & 1. 174707 & 3. 348491 & -0.447663 \\
\hline 36 & 1 & 0 & 0.630303 & 3. 940910 & 1. 555362 \\
\hline 37 & 1 & 0 & 1. 499055 & 2. 547565 & -2.426860 \\
\hline 38 & 1 & 0 & 1. 985921 & 4. 067054 & -0.521597 \\
\hline
\end{tabular}

TS21 in the reaction of adenine with 4-biphenylylnitrenium ion at B3PW91/6-31+G(d,p) 1eve1

Zero-point correction=

Thermal correction to Energy=

Thermal correction to Enthalpy=

Thermal correction to Gibbs Free Energy=

Sum of electronic and zero-point Energies=

Sum of electronic and thermal Energies=

Sum of electronic and thermal Enthalpies=

Sum of electronic and thermal Free Energies=
0. 302468 (Hartree/Particle)
0. 320153
0. 321097
0. 254607
$-984.451097$
$-984.433413$
$-984.432468$
$-984.498959$

\begin{tabular}{|c|c|c|c|c|c|}
\hline \multirow{2}{*}{$\begin{array}{l}\text { Center } \\
\text { Number }\end{array}$} & \multirow{2}{*}{$\begin{array}{l}\text { Atomic } \\
\text { Number }\end{array}$} & \multirow{2}{*}{$\begin{array}{l}\text { Atomic } \\
\text { Type }\end{array}$} & \multicolumn{3}{|c|}{ Coordinates (Angstroms) } \\
\hline & & & $\mathrm{X}$ & Y & Z \\
\hline 1 & 7 & 0 & 3. 241598 & -1.482898 & 1. 485653 \\
\hline 2 & 1 & 0 & 5. 227636 & -0.713568 & -0.363349 \\
\hline 3 & 7 & 0 & 2. 383449 & -0.550825 & -1.858748 \\
\hline 4 & 1 & 0 & 4. 302348 & -0.120137 & -2.663438 \\
\hline 5 & 6 & 0 & 2. 063849 & -0.959670 & -0.586719 \\
\hline 6 & 7 & 0 & -5.475181 & -1.316969 & -0.181878 \\
\hline 7 & 6 & 0 & 3.214453 & -1.085746 & 0.222033 \\
\hline 8 & 7 & 0 & 4. 245904 & -0728157 & -0.605304 \\
\hline
\end{tabular}




\begin{tabular}{rrrrrr}
9 & 6 & 0 & 2.030555 & -1.781970 & \multicolumn{1}{c}{1.962047} \\
10 & 7 & 0 & 0.857785 & -1.718934 & 1.305114 \\
11 & 6 & 0 & 0.877106 & -1.313922 & 0.043450 \\
12 & 1 & 0 & -1.979304 & 0.786597 & -2.201055 \\
13 & 6 & 0 & 3.690405 & -0.426333 & -1.824771 \\
14 & 1 & 0 & -5.960554 & -1.190380 & -1.074768 \\
15 & 6 & 0 & -4.281811 & -0.839897 & -0.220364 \\
16 & 6 & 0 & -1.558455 & 0.173717 & -0.123500 \\
17 & 6 & 0 & -3.656672 & -0.194112 & -1.381728 \\
18 & 6 & 0 & -3.457688 & -0.944976 & 0.987357 \\
19 & 6 & 0 & -2.201180 & -0.467631 & 1.041905 \\
20 & 6 & 0 & -2.393704 & 0.269380 & -1.340647 \\
21 & 1 & 0 & -4.255864 & -0.067427 & -2.280680 \\
22 & 1 & 0 & -3.919649 & -1.421591 & 1.846999 \\
23 & 1 & 0 & -1.607594 & -0.583686 & 1.942465 \\
24 & 7 & 0 & -0.370365 & -1.247019 & -0.642818 \\
25 & 1 & 0 & -0.947260 & -2.064008 & -0.439056 \\
26 & 1 & 0 & -0.225324 & -1.158267 & -1.649858 \\
27 & 1 & 0 & 1.982710 & -2.120355 & 2.992576 \\
28 & 6 & 0 & -0.653181 & 1.329187 & 0.124985 \\
29 & 6 & 0 & -0.579207 & 1.910560 & 1.402079 \\
30 & 6 & 0 & 0.085981 & 1.907559 & -0.923880 \\
31 & 6 & 0 & 0.200179 & 3.043988 & 1.619666 \\
32 & 1 & 0 & -1.157652 & 1.505214 & 2.224880 \\
33 & 6 & 0 & 0.872461 & 3.030735 & -0.700243 \\
34 & 1 & 0 & 0.072880 & 1.474145 & -1.919963 \\
35 & 6 & 0 & 0.927344 & 3.606203 & 0.571723 \\
36 & 1 & 0 & 0.231688 & 3.490817 & 2.608511 \\
37 & 1 & 0 & 1.438719 & 3.462058 & -1.520020 \\
38 & 1 & 0 & 1.532287 & 4.491788 & 0.742271
\end{tabular}

INT21 in the reaction of adenine with 4-biphenylylnitrenium ion at B3PW91/6-31+G(d,p) leve1

Zero-point correction=

Thermal correction to Energy=

Thermal correction to Enthalpy=

Thermal correction to Gibbs Free Energy=

Sum of electronic and zero-point Energies=

Sum of electronic and thermal Energies=

Sum of electronic and thermal Enthalpies=

Sum of electronic and thermal Free Energies=
0. 304173 (Hartree/Particle)
0. 321974
0. 322918
0. 256533
$-984.451681$
$-984.433880$
$-984.432935$
$-984.499321$

\begin{tabular}{|c|c|c|c|c|c|}
\hline \multirow{2}{*}{$\begin{array}{l}\text { Center } \\
\text { Number }\end{array}$} & \multirow{2}{*}{$\begin{array}{l}\text { Atomic } \\
\text { Number }\end{array}$} & \multirow{2}{*}{$\begin{array}{l}\text { Atomic } \\
\text { Type }\end{array}$} & \multicolumn{3}{|c|}{ Coordinates (Angstroms) } \\
\hline & & & $\mathrm{X}$ & Y & Z \\
\hline 1 & 7 & 0 & 3. 331727 & -1.327648 & 1. 564652 \\
\hline 2 & 1 & 0 & 5. 346244 & -0.612079 & -0.284115 \\
\hline 3 & 7 & 0 & 2. 517587 & -0.486840 & -1.813673 \\
\hline 4 & 1 & 0 & 4. 446339 & -0.081402 & -2.608390 \\
\hline 5 & 6 & 0 & 2. 185353 & -0.860668 & -0.535973 \\
\hline 6 & 7 & 0 & -5.403896 & -1.457556 & -0.156907 \\
\hline 7 & 6 & 0 & 3. 326601 & -0.968750 & 0.290343 \\
\hline 8 & 7 & 0 & 4. 366872 & -0637385 & -0.536224 \\
\hline
\end{tabular}




\begin{tabular}{rrrrrr}
9 & 6 & 0 & 2.114727 & -1.603400 & \multicolumn{1}{c}{2.037426} \\
10 & 7 & 0 & 0.949609 & -1.549656 & 1.360821 \\
11 & 6 & 0 & 0.996780 & -1.175078 & 0.099690 \\
12 & 1 & 0 & -1.891791 & 0.502227 & -2.292980 \\
13 & 6 & 0 & 3.825150 & -0.365283 & -1.768620 \\
14 & 1 & 0 & -5.909162 & -1.315112 & -1.035783 \\
15 & 6 & 0 & -4.197572 & -1.025669 & -0.231310 \\
16 & 6 & 0 & -1.409085 & -0.081017 & -0.207379 \\
17 & 6 & 0 & -3.578899 & -0.407031 & -1.413543 \\
18 & 6 & 0 & -3.338935 & -1.153786 & 0.952567 \\
19 & 6 & 0 & -2.070780 & -0.720010 & 0.980051 \\
20 & 6 & 0 & -2.304611 & 0.011054 & -1.413896 \\
21 & 1 & 0 & -4.194475 & -0.273728 & -2.300481 \\
22 & 1 & 0 & -3.791694 & -1.616592 & 1.824749 \\
23 & 1 & 0 & -1.460286 & -0.842105 & 1.868981 \\
24 & 7 & 0 & -0.257847 & -1.138649 & -0.656892 \\
25 & 1 & 0 & -0.709632 & -2.058641 & -0.612830 \\
26 & 1 & 0 & -0.017575 & -0.958993 & -1.638734 \\
27 & 1 & 0 & 2.050327 & -1.911580 & 3.076087 \\
28 & 6 & 0 & -0.732326 & 1.251644 & 0.073537 \\
29 & 6 & 0 & -0.961268 & 1.920734 & 1.281281 \\
30 & 6 & 0 & 0.066280 & 1.865821 & -0.903544 \\
31 & 6 & 0 & -0.395081 & 3.174253 & 1.511182 \\
32 & 1 & 0 & -1.597766 & 1.478371 & 2.040707 \\
33 & 6 & 0 & 0.637979 & 3.112870 & -0.667623 \\
34 & 1 & 0 & 0.261381 & 1.383852 & -1.859216 \\
35 & 6 & 0 & 0.407044 & 3.771475 & 0.541016 \\
36 & 1 & 0 & -0.587683 & 3.683803 & 2.450345 \\
37 & 1 & 0 & 1.254621 & 3.574404 & -1.432992 \\
38 & 1 & 0 & 0.844653 & 4.748772 & 0.721002
\end{tabular}

INT22 in the reaction of adenine with 4-biphenylylnitrenium ion at B3PW91/6-31+G(d,p) leve1

Zero-point correction=

Thermal correction to Energy=

Thermal correction to Enthalpy=

Thermal correction to Gibbs Free Energy=

Sum of electronic and zero-point Energies=

Sum of electronic and thermal Energies=

Sum of electronic and thermal Enthalpies=

Sum of electronic and thermal Free Energies=
0.303253 (Hartree/Particle)
0.321236
0.322180
0. 256323

$-984.472636$

$-984.454653$

$-984.453709$

$-984.519566$

\begin{tabular}{|c|c|c|c|c|c|}
\hline \multirow{2}{*}{$\begin{array}{l}\text { Center } \\
\text { Number }\end{array}$} & \multirow{2}{*}{$\begin{array}{l}\text { Atomic } \\
\text { Number }\end{array}$} & \multirow{2}{*}{$\begin{array}{l}\text { Atomic } \\
\text { Type }\end{array}$} & \multicolumn{3}{|c|}{ Coordinates (Angstroms) } \\
\hline & & & $X$ & $\mathrm{Y}$ & Z \\
\hline 1 & 7 & 0 & 3. 581519 & -0.106744 & 1. 302135 \\
\hline 2 & 1 & 0 & 5. 152037 & -1.619228 & -0.489298 \\
\hline 3 & 7 & 0 & 2. 065690 & -2.074001 & -1.274066 \\
\hline 4 & 1 & 0 & 3. 780751 & -2.913563 & -2.206014 \\
\hline 5 & 6 & 0 & 2. 011955 & -1.217187 & -0.201600 \\
\hline 6 & 7 & 0 & -4.408557 & -2.794659 & 0.258520 \\
\hline 7 & 6 & 0 & 3. 299842 & -0.904986 & 0.280258 \\
\hline 8 & 7 & 0 & 4. 142556 & -1.603502 & -0.543385 \\
\hline
\end{tabular}




$\begin{array}{rrrrrr}9 & 6 & 0 & 2.491712 & 0.397808 & 1.880366 \\ 10 & 7 & 0 & 1.208217 & 0.175173 & 1.535453 \\ 11 & 6 & 0 & 0.976055 & -0.615123 & 0.503752 \\ 12 & 1 & 0 & -2.319413 & 0.346577 & -2.230531 \\ 13 & 6 & 0 & 3.351996 & -2.278478 & -1.441701 \\ 14 & 1 & 0 & -5.073518 & -2.903348 & -0.489642 \\ 15 & 6 & 0 & -3.430330 & -1.856254 & 0.190603 \\ 16 & 6 & 0 & -1.437587 & 0.229134 & -0.215070 \\ 17 & 6 & 0 & -3.266222 & -1.170477 & -1.094939 \\ 18 & 6 & 0 & -2.616707 & -1.519400 & 1.240413 \\ 19 & 6 & 0 & -1.567997 & -0.532703 & 1.060753 \\ 20 & 6 & 0 & -2.357141 & -0.197781 & -1.292597 \\ 21 & 1 & 0 & -3.944504 & -1.434639 & -1.902825 \\ 22 & 1 & 0 & -2.745354 & -1.957206 & 2.224087 \\ 23 & 1 & 0 & -1.136586 & -0.068076 & 1.944193 \\ 24 & 7 & 0 & -0.373258 & -0.882757 & 0.126564 \\ 25 & 1 & 0 & -0.481503 & -1.723005 & -0.441896 \\ 26 & 1 & 0 & 2.648484 & 1.057223 & 2.728356 \\ 27 & 6 & 0 & -0.924736 & 1.627232 & -0.278107 \\ 28 & 6 & 0 & -1.257845 & 2.546805 & 0.721112 \\ 29 & 6 & 0 & -0.164074 & 2.041280 & -1.380006 \\ 30 & 6 & 0 & -0.828832 & 3.869107 & 0.622197 \\ 31 & 1 & 0 & -1.859105 & 2.236623 & 1.571013 \\ 32 & 6 & 0 & 0.273584 & 3.359791 & -1.468834 \\ 33 & 1 & 0 & 0.093402 & 1.334065 & -2.165568 \\ 34 & 6 & 0 & -0.060009 & 4.274892 & -0.468179 \\ 35 & 1 & 0 & -1.095150 & 4.581165 & 1.397278 \\ 36 & 1 & 0 & 0.869389 & 3.675221 & -2.320010 \\ 37 & 1 & 0 & 0.275783 & 5.304941 & -0.541999 \\ 38 & 1 & 0 & -4.639865 & -3.233275 & 1.135780\end{array}$

TS21 in the reaction of adenine with 4-biphenylylnitrenium ion at MP2/CC-PVDZ 1eve1

\begin{tabular}{|c|c|c|c|c|c|}
\hline \multirow{2}{*}{$\begin{array}{l}\text { Center } \\
\text { Number }\end{array}$} & \multirow{2}{*}{$\begin{array}{l}\text { Atomic } \\
\text { Number }\end{array}$} & \multirow{2}{*}{$\begin{array}{l}\text { Atomic } \\
\text { Type }\end{array}$} & \multicolumn{3}{|c|}{ Coordinates (Angstroms) } \\
\hline & & & $X$ & $Y$ & Z \\
\hline 1 & 7 & 0 & 2. 916085 & -1.744227 & 0.786062 \\
\hline 2 & 1 & 0 & 4. 821578 & -0.097501 & -0.520218 \\
\hline 3 & 7 & 0 & 1. 990427 & 0.261142 & -2.067869 \\
\hline 4 & 1 & 0 & 3. 872846 & 1. 202927 & -2.508172 \\
\hline 5 & 6 & 0 & 1.716935 & -0.630323 & -1.054409 \\
\hline 6 & 7 & 0 & -5.723673 & -0.399657 & -0.219698 \\
\hline 7 & 6 & 0 & 2. 856140 & -0.905910 & -0.255783 \\
\hline 8 & 7 & 0 & 3. 846589 & -0.127407 & -0.811147 \\
\hline 9 & 6 & 0 & 1. 734306 & -2.349252 & 1. 012283 \\
\hline 10 & 7 & 0 & 0.558303 & -2.175604 & 0.349445 \\
\hline 11 & 6 & 0 & 0.553391 & -1.321140 & -0.679960 \\
\hline 12 & 1 & 0 & -1.974823 & 1. 978237 & -1.371108 \\
\hline 13 & 6 & 0 & 3. 284684 & 0.531107 & -1.880985 \\
\hline 14 & 1 & 0 & -6.188240 & 0.225088 & -0.901540 \\
\hline 15 & 6 & 0 & -4.450606 & -0.109119 & -0.161300 \\
\hline 16 & 6 & 0 & -1.611032 & 0. 388128 & 0.124952 \\
\hline
\end{tabular}




$\begin{array}{rrrrrr}17 & 6 & 0 & -3.774187 & 0.932568 & -0.942376 \\ 18 & 6 & 0 & -3.622005 & -0.898132 & 0.755407 \\ 19 & 6 & 0 & -2.284599 & -0.655717 & 0.898278 \\ 20 & 6 & 0 & -2.434582 & 1.166827 & -0.801830 \\ 21 & 1 & 0 & -4.372528 & 1.553507 & -1.620111 \\ 22 & 1 & 0 & -4.127090 & -1.681070 & 1.330739 \\ 23 & 1 & 0 & -1.685775 & -1.287537 & 1.557694 \\ 24 & 7 & 0 & -0.683706 & -1.002424 & -1.261429 \\ 25 & 1 & 0 & -1.346699 & -1.785567 & -1.261378 \\ 26 & 1 & 0 & -0.587805 & -0.574633 & -2.190051 \\ 27 & 1 & 0 & 1.709016 & -3.069192 & 1.836383 \\ 28 & 6 & 0 & -0.368860 & 0.980497 & 0.636338 \\ 29 & 6 & 0 & 0.199716 & 0.529145 & 1.859287 \\ 30 & 6 & 0 & 0.327519 & 1.969927 & -0.111634 \\ 31 & 6 & 0 & 1.414825 & 1.056150 & 2.316701 \\ 32 & 1 & 0 & -0.324228 & -0.200344 & 2.480180 \\ 33 & 6 & 0 & 1.535640 & 2.497597 & 0.358428 \\ 34 & 1 & 0 & -0.045230 & 2.307458 & -1.081683 \\ 35 & 6 & 0 & 2.089760 & 2.038224 & 1.568301 \\ 36 & 1 & 0 & 1.832737 & 0.703180 & 3.264557 \\ 37 & 1 & 0 & 2.051424 & 3.264962 & -0.227402 \\ 38 & 1 & 0 & 3.034371 & 2.454261 & 1.934377\end{array}$

S-COM21 in the reaction of adenine with 4-biphenylylnitrenium ion at B3PW91/6-31+G(d,p) 1 eve1

Zero-point correction=

Thermal correction to Energy=

Thermal correction to Enthalpy=

Thermal correction to Gibbs Free Energy=

Sum of electronic and zero-point Energies=

Sum of electronic and thermal Energies=

Sum of electronic and thermal Enthalpies=

Sum of electronic and thermal Free Energies=

\begin{tabular}{|c|c|c|c|c|c|}
\hline \multirow{2}{*}{$\begin{array}{l}\text { Center } \\
\text { Number }\end{array}$} & \multirow{2}{*}{$\begin{array}{l}\text { Atomic } \\
\text { Number }\end{array}$} & \multirow{2}{*}{$\begin{array}{l}\text { Atomic } \\
\text { Type }\end{array}$} & \multicolumn{3}{|c|}{ Coordinates (Angstroms) } \\
\hline & & & $X$ & Y & Z \\
\hline 1 & 7 & 0 & -3.882661 & -0.718154 & 0.440527 \\
\hline 2 & 1 & 0 & -4.926340 & 1. 319065 & -1.193162 \\
\hline 3 & 7 & 0 & -2.120290 & 2. 359972 & -0.020679 \\
\hline 4 & 1 & 0 & -3.450703 & 3. 398278 & -1.316838 \\
\hline 5 & 6 & 0 & -2.252562 & 1. 099866 & 0.509264 \\
\hline 6 & 7 & 0 & -2.042657 & -2.130162 & -1.522520 \\
\hline 7 & 6 & 0 & -3.444361 & 0.488518 & 0.090363 \\
\hline 8 & 7 & 0 & -4.035719 & 1. 419068 & -0.725788 \\
\hline 9 & 6 & 0 & -3.049117 & -1.323762 & 1. 297122 \\
\hline 10 & 7 & 0 & -1.888039 & -0.873524 & 1. 785798 \\
\hline 11 & 6 & 0 & -1.465666 & 0.350439 & 1. 413311 \\
\hline 12 & 1 & 0 & 1. 860847 & -2.311675 & 0.817415 \\
\hline 13 & 6 & 0 & -3.205916 & 2. 510814 & -0.747958 \\
\hline 14 & 1 & 0 & -2.274820 & -3.019537 & -1.069398 \\
\hline 15 & 6 & 0 & -0.886264 & -1.682954 & -1.140705 \\
\hline 16 & 6 & 0 & 1. 672906 & -0.587908 & -0.507106 \\
\hline
\end{tabular}

0. 301138 (Hartree/Particle)

0. 320185

0. 321129

0. 251272

$-984.467231$

$-984.448184$

$-984.447240$

$-984.517097$ 


$\begin{array}{rrrrrr}17 & 6 & 0 & 0.000804 & -2.333553 & -0.185021 \\ 18 & 6 & 0 & -0.428685 & -0.428978 & -1.719079 \\ 19 & 6 & 0 & 0.795224 & 0.086338 & -1.412405 \\ 20 & 6 & 0 & 1.227009 & -1.813996 & 0.092535 \\ 21 & 1 & 0 & -0.334871 & -3.247008 & 0.297907 \\ 22 & 1 & 0 & -1.085707 & 0.050127 & -2.438008 \\ 23 & 1 & 0 & 1.124378 & 0.997112 & -1.898596 \\ 24 & 7 & 0 & -0.290415 & 0.805476 & 1.899098 \\ 25 & 1 & 0 & 0.101550 & 0.317359 & 2.692073 \\ 26 & 1 & 0 & -0.079006 & 1.788022 & 1.798543 \\ 27 & 1 & 0 & -3.360092 & -2.304551 & 1.649645 \\ 28 & 6 & 0 & 2.997670 & -0.066524 & -0.229065 \\ 29 & 6 & 0 & 3.296685 & 1.305161 & -0.418744 \\ 30 & 6 & 0 & 4.032788 & -0.921349 & 0.222760 \\ 31 & 6 & 0 & 4.565277 & 1.796656 & -0.155696 \\ 32 & 1 & 0 & 2.520761 & 1.995517 & -0.732068 \\ 33 & 6 & 0 & 5.306589 & -0.428270 & 0.460229 \\ 34 & 1 & 0 & 3.853146 & -1.984692 & 0.339076 \\ 35 & 6 & 0 & 5.575802 & 0.931699 & 0.278556 \\ 36 & 1 & 0 & 4.773067 & 2.853946 & -0.286443 \\ 37 & 1 & 0 & 6.094989 & -1.101397 & 0.781653 \\ 38 & 1 & 0 & 6.572183 & 1.317532 & 0.472999\end{array}$

S-TS21 in the reaction of adenine with 4-biphenylylnitrenium ion at B3PW91/6-31+G(d,p) leve1

Zero-point correction=

Thermal correction to Energy=

Thermal correction to Enthalpy=

Thermal correction to Gibbs Free Energy=

Sum of electronic and zero-point Energies=

Sum of electronic and thermal Energies=

Sum of electronic and thermal Enthalpies=

Sum of electronic and thermal Free Energies=

\begin{tabular}{|c|c|c|c|c|c|}
\hline \multirow{2}{*}{$\begin{array}{l}\text { Center } \\
\text { Number }\end{array}$} & \multirow{2}{*}{$\begin{array}{l}\text { Atomic } \\
\text { Number }\end{array}$} & \multirow{2}{*}{$\begin{array}{l}\text { Atomic } \\
\text { Type }\end{array}$} & \multicolumn{3}{|c|}{ Coordinates (Angstroms) } \\
\hline & & & $X$ & $\mathrm{Y}$ & Z \\
\hline 1 & 6 & 0 & -5.163113 & -1.248326 & 0.016695 \\
\hline 2 & 6 & 0 & -4.542506 & -0.961800 & -1.197768 \\
\hline 3 & 6 & 0 & -3.263309 & -0.410336 & -1.220330 \\
\hline 4 & 6 & 0 & -2.588232 & -0.123666 & -0.022312 \\
\hline 5 & 6 & 0 & -3.234160 & -0.404271 & 1. 197402 \\
\hline 6 & 6 & 0 & -4.504011 & -0.968849 & 1. 216203 \\
\hline 7 & 6 & 0 & -1.226215 & 0.493795 & -0.041933 \\
\hline 8 & 6 & 0 & -0.548407 & 0.638674 & -1.341889 \\
\hline 9 & 6 & 0 & 0.248979 & 1. 686150 & -1.614776 \\
\hline 10 & 6 & 0 & 0.470086 & 2. 767592 & -0.650922 \\
\hline 11 & 6 & 0 & -0.221162 & 2. 643862 & 0.637134 \\
\hline 12 & 6 & 0 & -0.998536 & 1. 584699 & 0.925109 \\
\hline 13 & 7 & 0 & 1. 246401 & 3. 733025 & -0.996409 \\
\hline 14 & 7 & 0 & -0.222943 & -0.956264 & 0.713977 \\
\hline 15 & 6 & 0 & 1. 178405 & -0.773749 & 0.863260 \\
\hline 16 & 6 & 0 & 2. 076777 & -1.155573 & -0.127620 \\
\hline
\end{tabular}

0. 302589 (Hartree/Particle)

0. 320218

0.321162

0. 255058

$-984.452980$

$-984.435351$

$-984.434407$

$-984.500511$ 


$\begin{array}{rrrrrr}17 & 6 & 0 & 3.430076 & -0.898024 & 0.185322 \\ 18 & 7 & 0 & 3.883272 & -0.331623 & 1.294193 \\ 19 & 6 & 0 & 2.910075 & -0.011539 & 2.150396 \\ 20 & 7 & 0 & 1.588489 & -0.204723 & 1.989554 \\ 21 & 7 & 0 & 4.121323 & -1.378220 & -0.895388 \\ 22 & 6 & 0 & 3.191027 & -1.889325 & -1.766383 \\ 23 & 7 & 0 & 1.951317 & -1.776348 & -1.346886 \\ 24 & 1 & 0 & 5.125458 & -1.367072 & -1.014515 \\ 25 & 1 & 0 & 3.484677 & -2.340359 & -2.705510 \\ 26 & 1 & 0 & -1.515673 & 1.541453 & 1.877789 \\ 27 & 1 & 0 & 1.326696 & 4.433185 & -0.253125 \\ 28 & 1 & 0 & -0.106239 & 3.449033 & 1.359114 \\ 29 & 1 & 0 & 0.750652 & 1.781400 & -2.573177 \\ 30 & 1 & 0 & -0.676949 & -0.149642 & -2.076804 \\ 31 & 1 & 0 & -0.661682 & -1.103499 & 1.623125 \\ 32 & 1 & 0 & -0.447555 & -1.728664 & 0.085041 \\ 33 & 1 & 0 & 3.214886 & 0.450155 & 3.084566 \\ 34 & 1 & 0 & -2.811739 & -0.173689 & -2.177542 \\ 35 & 1 & 0 & -2.754560 & -0.181998 & 2.148036 \\ 36 & 1 & 0 & -5.054946 & -1.162691 & -2.133459 \\ 37 & 1 & 0 & -4.983913 & -1.183343 & 2.166070 \\ 38 & 1 & 0 & -6.158766 & -1.681090 & 0.031580\end{array}$

S-INT21 in the reaction of adenine with 4-biphenylylnitrenium ion at B3PW91/6-31+G(d,p) leve1

Zero-point correction=

Thermal correction to Energy=

Thermal correction to Enthalpy=

Thermal correction to Gibbs Free Energy=

Sum of electronic and zero-point Energies=

Sum of electronic and thermal Energies=

Sum of electronic and thermal Enthalpies=

Sum of electronic and thermal Free Energies=

\begin{tabular}{|c|c|c|c|c|c|}
\hline \multirow{2}{*}{$\begin{array}{l}\text { Center } \\
\text { Number }\end{array}$} & \multirow{2}{*}{$\begin{array}{l}\text { Atomic } \\
\text { Number }\end{array}$} & \multirow{2}{*}{$\begin{array}{l}\text { Atomic } \\
\text { Type }\end{array}$} & \multicolumn{3}{|c|}{ Coordinates (Angstroms) } \\
\hline & & & $X$ & Y & Z \\
\hline 1 & 6 & 0 & -5.073947 & -1.369240 & -0.136130 \\
\hline 2 & 6 & 0 & -4.505568 & -0.753926 & -1.249228 \\
\hline 3 & 6 & 0 & -3.209883 & -0.240930 & -1.186468 \\
\hline 4 & 6 & 0 & -2.469028 & -0.334504 & -0.003196 \\
\hline 5 & 6 & 0 & -3.057384 & -0.939303 & 1. 120003 \\
\hline 6 & 6 & 0 & -4.346668 & -1.459929 & 1. 051543 \\
\hline 7 & 6 & 0 & -1.056228 & 0.242479 & 0.085057 \\
\hline 8 & 6 & 0 & -0.490629 & 0.704749 & -1.223321 \\
\hline 9 & 6 & 0 & -0.015579 & 1.943435 & -1.406695 \\
\hline 10 & 6 & 0 & -0.000885 & 2.948633 & -0.336624 \\
\hline 11 & 6 & 0 & -0.500392 & 2.505857 & 0.972584 \\
\hline 12 & 6 & 0 & -0.949537 & 1. 260779 & 1. 182542 \\
\hline 13 & 7 & 0 & 0.453952 & 4. 116951 & -0.613106 \\
\hline 14 & 7 & 0 & -0.153232 & -1.032502 & 0.509855 \\
\hline 15 & 6 & 0 & 1. 274326 & -0.811752 & 0.718176 \\
\hline 16 & 6 & 0 & 2. 191972 & -1.027009 & -0.296443 \\
\hline
\end{tabular}

0. 304106 (Hartree/Particle)

0. 321927

0. 322871

0. 256128

$-984.453876$

$-984.436055$

$-984.435111$

$-984.501854$ 


$\begin{array}{rrrrrr}17 & 6 & 0 & 3.530520 & -0.772804 & 0.086158 \\ 18 & 7 & 0 & 3.938187 & -0.363292 & 1.277350 \\ 19 & 6 & 0 & 2.941806 & -0.208559 & 2.150967 \\ 20 & 7 & 0 & 1.630050 & -0.419887 & 1.925833 \\ 21 & 7 & 0 & 4.258063 & -1.063133 & -1.036550 \\ 22 & 6 & 0 & 3.363881 & -1.471072 & -1.995430 \\ 23 & 7 & 0 & 2.112698 & -1.464670 & -1.595027 \\ 24 & 1 & 0 & 5.263151 & -0.997879 & -1.130428 \\ 25 & 1 & 0 & 3.691621 & -1.767795 & -2.983514 \\ 26 & 1 & 0 & -1.317925 & 0.974795 & 2.163765 \\ 27 & 1 & 0 & 0.417844 & 4.734709 & 0.202483 \\ 28 & 1 & 0 & -0.500673 & 3.226375 & 1.787301 \\ 29 & 1 & 0 & 0.375649 & 2.259727 & -2.369335 \\ 30 & 1 & 0 & -0.476324 & -0.013921 & -2.039457 \\ 31 & 1 & 0 & -0.545981 & -1.408894 & 1.378417 \\ 32 & 1 & 0 & -0.293088 & -1.735222 & -0.224809 \\ 33 & 1 & 0 & 3.210298 & 0.114283 & 3.151728 \\ 34 & 1 & 0 & -2.790622 & 0.251435 & -2.057625 \\ 35 & 1 & 0 & -2.534394 & -0.991939 & 2.074547 \\ 36 & 1 & 0 & -5.069777 & -0.667750 & -2.172790 \\ 37 & 1 & 0 & -4.786531 & -1.924527 & 1.928695 \\ 38 & 1 & 0 & -6.081922 & -1.769100 & -0.188433\end{array}$

S-INT22 in the reaction of adenine with 4-biphenylylnitrenium ion at B3PW91/6-31+G(d,p) leve1

Zero-point correction=

Thermal correction to Energy=

Thermal correction to Enthalpy=

Thermal correction to Gibbs Free Energy=

Sum of electronic and zero-point Energies=

Sum of electronic and thermal Energies=

Sum of electronic and thermal Enthalpies=

Sum of electronic and thermal Free Energies=

\begin{tabular}{cccccc}
$\begin{array}{c}\text { Center } \\
\text { Number }\end{array}$ & $\begin{array}{c}\text { Atomic } \\
\text { Number }\end{array}$ & $\begin{array}{c}\text { Atomic } \\
\text { Type }\end{array}$ & \multicolumn{3}{c}{ Coordinates (Angstroms) } \\
------------------------ & $\mathrm{X}$ & $\mathrm{Y}$ & $Z$ \\
1 & 7 & 0 & 3.948751 & -0.779435 & -0.805448 \\
2 & 1 & 0 & 4.090353 & -2.988798 & 0.925580 \\
3 & 7 & 0 & 1.001900 & -2.110586 & 0.720359 \\
4 & 1 & 0 & 1.825968 & -3.711002 & 1.847998 \\
5 & 6 & 0 & 1.650066 & -1.206282 & -0.088736 \\
6 & 7 & 0 & 1.488298 & 3.736552 & 1.256639 \\
7 & 6 & 0 & 3.043994 & -1.451432 & -0.111655 \\
8 & 7 & 0 & 3.213753 & -2.530644 & 0.715846 \\
9 & 6 & 0 & 3.419092 & 0.204762 & -1.535288 \\
10 & 7 & 0 & 2.120131 & 0.545754 & -1.612464 \\
11 & 6 & 0 & 1.250519 & -0.147387 & -0.898154 \\
12 & 1 & 0 & -0.830756 & -0.044341 & 2.144508 \\
13 & 6 & 0 & 1.968202 & -2.875799 & 1.174099 \\
14 & 1 & 0 & 1.699304 & 3.811453 & 2.238415 \\
15 & 6 & 0 & 0.649919 & 2.771498 & 0.798826 \\
16 & 6 & 0 & -1.115447 & 0.585874 & 0.055683
\end{tabular}

0. 303210 (Hartree/Particle)

0.321136

0.322080

0.256477

$-984.464998$

$-984.447072$

$-984.446128$

$-984.511731$ 


$\begin{array}{rrrrrr}17 & 6 & 0 & 0.283650 & 1.714461 & 1.742487 \\ 18 & 6 & 0 & 0.144447 & 2.746279 & -0.474689 \\ 19 & 6 & 0 & -0.714781 & 1.667198 & -0.896140 \\ 20 & 6 & 0 & -0.527786 & 0.695218 & 1.411742 \\ 21 & 1 & 0 & 0.663414 & 1.783895 & 2.759233 \\ 22 & 1 & 0 & 0.365958 & 3.531997 & -1.187776 \\ 23 & 1 & 0 & -1.362886 & 1.849356 & -1.748352 \\ 24 & 7 & 0 & -0.138129 & 0.198396 & -1.066526 \\ 25 & 1 & 0 & -0.609132 & -0.317217 & -1.808622 \\ 26 & 1 & 0 & 4.105667 & 0.791856 & -2.137348 \\ 27 & 6 & 0 & -2.444593 & -0.094173 & -0.055837 \\ 28 & 6 & 0 & -2.544481 & -1.471260 & 0.187123 \\ 29 & 6 & 0 & -3.590703 & 0.648083 & -0.360172 \\ 30 & 6 & 0 & -3.785389 & -2.098553 & 0.106519 \\ 31 & 1 & 0 & -1.652900 & -2.043219 & 0.436104 \\ 32 & 6 & 0 & -4.831229 & 0.014703 & -0.430327 \\ 33 & 1 & 0 & -3.522171 & 1.719863 & -0.528115 \\ 34 & 6 & 0 & -4.928267 & -1.357304 & -0.201215 \\ 35 & 1 & 0 & -3.861862 & -3.166525 & 0.287543 \\ 36 & 1 & 0 & -5.719604 & 0.593846 & -0.663048 \\ 37 & 1 & 0 & -5.894475 & -1.849477 & -0.259404 \\ 38 & 1 & 0 & 1.696922 & 4.542725 & 0.688846\end{array}$

IMINE in the reaction of adenine with 4-biphenylylnitrenium ion at B3PW91/6-31+G(d,p) leve1

Zero-point correction=

Thermal correction to Energy=

Thermal correction to Enthalpy=

Thermal correction to Gibbs Free Energy=

Sum of electronic and zero-point Energies=

Sum of electronic and thermal Energies=

Sum of electronic and thermal Enthalpies=

Sum of electronic and thermal Free Energies=

\begin{tabular}{|c|c|c|c|c|c|}
\hline \multirow{2}{*}{$\begin{array}{l}\text { Center } \\
\text { Number }\end{array}$} & \multirow{2}{*}{$\begin{array}{l}\text { Atomic } \\
\text { Number }\end{array}$} & \multirow{2}{*}{$\begin{array}{l}\text { Atomic } \\
\text { Type }\end{array}$} & \multicolumn{3}{|c|}{ Coordinates (Angstroms) } \\
\hline & & & $\mathrm{X}$ & $\mathrm{Y}$ & Z \\
\hline 1 & 7 & 0 & 3. 725797 & 0.552103 & -1.106930 \\
\hline 2 & 1 & 0 & 5. 240549 & -0.901458 & 0.739740 \\
\hline 3 & 7 & 0 & 2. 171229 & -1.745778 & 1. 162231 \\
\hline 4 & 1 & 0 & 3. 862309 & -2.457349 & 2. 230653 \\
\hline 5 & 6 & 0 & 2. 119596 & -0.822519 & 0.132879 \\
\hline 6 & 7 & 0 & -3.328409 & -3.821858 & -0.188118 \\
\hline 7 & 6 & 0 & 3. 403408 & -0.346707 & -0.176556 \\
\hline 8 & 7 & 0 & 4. 238601 & -1.006279 & 0.691000 \\
\hline 9 & 6 & 0 & 2. 642846 & 0.983462 & -1.758454 \\
\hline 10 & 7 & 0 & 1. 366062 & 0.621981 & -1.584760 \\
\hline 11 & 6 & 0 & 1. 068772 & -0.287596 & -0.643084 \\
\hline 12 & 1 & 0 & -3.081692 & 0.464010 & -1.649832 \\
\hline 13 & 6 & 0 & 3. 443241 & -1.823374 & 1. 460108 \\
\hline 14 & 1 & 0 & -4.306123 & -3.784607 & -0.440568 \\
\hline 15 & 6 & 0 & -2.674181 & -2.594618 & -0.111645 \\
\hline 16 & 6 & 0 & -1.425442 & 0.040331 & -0.248445 \\
\hline
\end{tabular}

0.289964 (Hartree/Particle)

0. 307613

0. 308557

0. 243122

$-984.088627$

$-984.070977$

$-984.070033$

$-984.135469$ 


$\begin{array}{rrrrrr}17 & 6 & 0 & -3.146922 & -1.555107 & -1.021810 \\ 18 & 6 & 0 & -1.663286 & -2.335343 & 0.756169 \\ 19 & 6 & 0 & -0.912024 & -1.074215 & 0.677554 \\ 20 & 6 & 0 & -2.616969 & -0.318551 & -1.055945 \\ 21 & 1 & 0 & -4.009231 & -1.784789 & -1.644955 \\ 22 & 1 & 0 & -1.382033 & -3.050210 & 1.524862 \\ 23 & 1 & 0 & -0.403828 & -0.759107 & 1.587090 \\ 24 & 7 & 0 & -0.220007 & -0.739513 & -0.551427 \\ 25 & 1 & 0 & -3.154067 & -4.456021 & 0.578841 \\ 26 & 1 & 0 & 2.819005 & 1.724768 & -2.535069 \\ 27 & 6 & 0 & -1.321955 & 1.481420 & 0.163847 \\ 28 & 6 & 0 & -1.076077 & 2.475421 & -0.793300 \\ 29 & 6 & 0 & -1.540489 & 1.862332 & 1.492500 \\ 30 & 6 & 0 & -1.037228 & 3.818107 & -0.423594 \\ 31 & 1 & 0 & -0.887933 & 2.187713 & -1.822709 \\ 32 & 6 & 0 & -1.499111 & 3.207039 & 1.863561 \\ 33 & 1 & 0 & -1.756054 & 1.104306 & 2.240436 \\ 34 & 6 & 0 & -1.246312 & 4.188146 & 0.906148 \\ 35 & 1 & 0 & -0.836351 & 4.577136 & -1.174886 \\ 36 & 1 & 0 & -1.670539 & 3.485865 & 2.899773 \\ 37 & 1 & 0 & -1.214118 & 5.235765 & 1.193060\end{array}$

AZEPINE in the reaction of adenine with 4-biphenylylnitrenium ion at B3PW91/6-31+G(d,p) leve1

Zero-point correction=

Thermal correction to Energy=

Thermal correction to Enthalpy=

Thermal correction to Gibbs Free Energy=

Sum of electronic and zero-point Energies=

Sum of electronic and thermal Energies=

Sum of electronic and thermal Enthalpies=

Sum of electronic and thermal Free Energies=

$\begin{array}{ccc}\text { Center } & \text { Atomic } & \text { Atomic } \\ \text { Number } & \text { Number } & \text { Type }\end{array}$

0. 289774 (Hartree/Particle)

0. 307788

0. 308732

0.242654

$-984.108329$

$-984.090315$

$-984.089371$

$-984.155448$

X Coordinates (Angstroms)

$\begin{array}{rrrrrr}1 & 7 & 0 & 2.156987 & -2.596977 & 1.515524 \\ 2 & 1 & 0 & 4.561206 & -2.790462 & 0.125137 \\ 3 & 7 & 0 & 2.924795 & -0.358933 & -1.179728 \\ 4 & 1 & 0 & 4.839891 & -1.073763 & -1.753906 \\ 5 & 6 & 0 & 2.090571 & -0.811060 & -0.170863 \\ 6 & 7 & 0 & 0.991291 & 4.705464 & 0.383832 \\ 7 & 6 & 0 & 2.672615 & -1.895241 & 0.503977 \\ 8 & 7 & 0 & 3.882993 & -2.083869 & -0.115634 \\ 9 & 6 & 0 & 0.948531 & -2.138920 & 1.848796 \\ 10 & 7 & 0 & 0.267821 & -1.122521 & 1.311215 \\ 11 & 6 & 0 & 0.809867 & -0.429137 & 0.298242 \\ 12 & 1 & 0 & -2.655468 & 2.144788 & 1.026055 \\ 13 & 6 & 0 & 3.973141 & -1.139549 & -1.109495 \\ 14 & 1 & 0 & 0.544340 & 5.404807 & 0.957749 \\ 15 & 6 & 0 & 0.284097 & 3.537262 & 0.137474 \\ 16 & 6 & 0 & -1.279331 & 0.830504 & 0.175609 \\ 17 & 6 & 0 & -0.772955 & 3.166701 & 0.927536\end{array}$




$\begin{array}{rrrrrr}18 & 6 & 0 & 0.697067 & 2.793564 & -1.043626 \\ 19 & 6 & 0 & 0.590791 & 1.466390 & -1.220719 \\ 20 & 6 & 0 & -1.614988 & 2.020471 & 0.732979 \\ 21 & 1 & 0 & -1.091199 & 3.872457 & 1.694423 \\ 22 & 1 & 0 & 1.119206 & 3.367030 & -1.867781 \\ 23 & 1 & 0 & 0.917891 & 1.000013 & -2.146576 \\ 24 & 7 & 0 & 0.084912 & 0.610580 & -0.214879 \\ 25 & 1 & 0 & 1.535400 & 5.085974 & -0.374971 \\ 26 & 1 & 0 & 0.449995 & -2.655052 & 2.666484 \\ 27 & 6 & 0 & -2.254093 & -0.236147 & -0.131243 \\ 28 & 6 & 0 & -3.399979 & -0.423553 & 0.659338 \\ 29 & 6 & 0 & -2.065396 & -1.087701 & -1.231133 \\ 30 & 6 & 0 & -4.332752 & -1.407547 & 0.345522 \\ 31 & 1 & 0 & -3.543912 & 0.184459 & 1.547665 \\ 32 & 6 & 0 & -2.998189 & -2.073470 & -1.543891 \\ 33 & 1 & 0 & -1.183213 & -0.968142 & -1.853816 \\ 34 & 6 & 0 & -4.140681 & -2.237263 & -0.760675 \\ 35 & 1 & 0 & -5.206964 & -1.537506 & 0.978165 \\ 36 & 1 & 0 & -2.831516 & -2.714606 & -2.405575 \\ 37 & 1 & 0 & -4.867148 & -3.008028 & -1.002345 \\ & & & & & \end{array}$

IMINE in the reaction of adenine with 4-biphenylylnitrenium ion at B3LYP/6-31+G(d,p) 1 eve1

\section{Zero-point correction=}

Thermal correction to Energy=

Thermal correction to Enthalpy=

Thermal correction to Gibbs Free Energy=

Sum of electronic and zero-point Energies=

Sum of electronic and thermal Energies=

Sum of electronic and thermal Enthalpies=

Sum of electronic and thermal Free Energies=

$\begin{array}{cc}\text { Center } & \text { Aton } \\ \text { Number } & \text { Numb } \\ --------- & 7 \\ 1 & 1 \\ 2 & 7 \\ 3 & 1 \\ 4 & 6 \\ 5 & 7 \\ 6 & 6 \\ 7 & 7 \\ 8 & 6 \\ 9 & 7 \\ 10 & 6 \\ 11 & 1 \\ 12 & 6 \\ 13 & 1 \\ 14 & 6 \\ 15 & 6 \\ 16 & 6 \\ 17 & 6 \\ 18 & \end{array}$

0. 288629 (Hartree/Particle)

0. 306307

0.307251

0. 241704

$-984.466001$

$-984.448323$

$-984.447379$

$-984.512926$

Coordinates (Angstroms)

X Y Z Z

$\begin{array}{rrr}3.737392 & 0.583399 & -1.106742 \\ 5.265188 & -0.863724 & 0.746701 \\ 2.195493 & -1.730537 & 1.165237 \\ 3.890771 & -2.432537 & 2.237631 \\ 2.137750 & -0.803769 & 0.132033 \\ -3.308877 & -3.857932 & -0.186337 \\ 3.421431 & -0.319645 & -0.174432 \\ 4.263064 & -0.975566 & 0.696443 \\ 2.651170 & 1.009806 & -1.762765 \\ 1.374188 & 0.637250 & -1.589548 \\ 1.081397 & -0.277506 & -0.646084 \\ -3.085191 & 0.432749 & -1.662080 \\ 3.469009 & -1.801399 & 1.466954 \\ -4.290498 & -3.826604 & -0.429456 \\ -2.659494 & -2.621689 & -0.111284 \\ -1.428579 & 0.028447 & -0.249577 \\ -3.142154 & -1.584134 & -1.024535 \\ -1.645062 & -2.357684 & 0.752190\end{array}$




$\begin{array}{rrrrrr}19 & 6 & 0 & -0.902702 & -1.085956 & 0.675381 \\ 20 & 6 & 0 & -2.618524 & -0.343648 & -1.062637 \\ 21 & 1 & 0 & -4.001736 & -1.822561 & -1.647370 \\ 22 & 1 & 0 & -1.354706 & -3.072268 & 1.517424 \\ 23 & 1 & 0 & -0.397211 & -0.771795 & 1.585740 \\ 24 & 7 & 0 & -0.207538 & -0.742313 & -0.557812 \\ 25 & 1 & 0 & -3.123669 & -4.494127 & 0.578099 \\ 26 & 1 & 0 & 2.823530 & 1.752102 & -2.538213 \\ 27 & 6 & 0 & -1.342308 & 1.474629 & 0.164558 \\ 28 & 6 & 0 & -1.112505 & 2.475028 & -0.793673 \\ 29 & 6 & 0 & -1.561814 & 1.853012 & 1.496422 \\ 30 & 6 & 0 & -1.092030 & 3.820200 & -0.422807 \\ 31 & 1 & 0 & -0.922849 & 2.192259 & -1.823481 \\ 32 & 6 & 0 & -1.538757 & 3.200384 & 1.868951 \\ 33 & 1 & 0 & -1.762445 & 1.092819 & 2.245407 \\ 34 & 6 & 0 & -1.303037 & 4.187444 & 0.909994 \\ 35 & 1 & 0 & -0.904230 & 4.581944 & -1.174214 \\ 36 & 1 & 0 & -1.710538 & 3.475574 & 2.905702 \\ 37 & 1 & 0 & -1.285214 & 5.234934 & 1.197265 \\ & & & & & \end{array}$

\section{AZEPINE in the reaction of adenine with 4-biphenylylnitrenium ion at B3LYP/6-31+G(d,p) leve1}

\begin{tabular}{|c|c|c|c|c|c|}
\hline & & & & & \\
\hline Zero-point correction $=$ & & & 0.288628 & rtree/Part & cle) \\
\hline Thermal correction to Er & $g y=$ & & 0.306622 & & \\
\hline Thermal correction to Er & lalpy= & & 0.307566 & & \\
\hline Thermal correction to G & s Free Er & & 0.241605 & & \\
\hline Sum of electronic and $\mathrm{z}$ & -point Er & ies $=$ & -984.4 & 9995 & \\
\hline Sum of electronic and tl & mal Eners & & -984.4 & 2002 & \\
\hline Sum of electronic and tl & mal Enthe & es $=$ & -984.4 & 1057 & \\
\hline Sum of electronic and tl & mal Free & rgies= & -984.5 & 7018 & \\
\hline Center & Atomic & Atomic & & ordinates & Angstroms) \\
\hline Number & Number & Type & $\mathrm{X}$ & $\mathrm{Y}$ & Z \\
\hline 1 & 7 & 0 & 2. 180682 & -2.585217 & 1. 528392 \\
\hline 2 & 1 & 0 & 4. 585207 & -2.782894 & 0.124114 \\
\hline 3 & 7 & 0 & 2. 930703 & -0.362824 & -1.193401 \\
\hline 4 & 1 & 0 & 4. 846866 & -1.075750 & -1.773999 \\
\hline 5 & 6 & 0 & 2. 099358 & -0.810231 & -0.172357 \\
\hline 6 & 7 & 0 & 0.969778 & 4. 726089 & 0.386312 \\
\hline 7 & 6 & 0 & 2. 691594 & -1.888534 & 0.507455 \\
\hline 8 & 7 & 0 & 3. 903125 & -2.079222 & -0.118479 \\
\hline 9 & 6 & 0 & 0.968763 & -2.130721 & 1. 865864 \\
\hline 10 & 7 & 0 & 0.280923 & -1.118500 & 1. 321551 \\
\hline 11 & 6 & 0 & 0.816712 & -0.427433 & 0.298674 \\
\hline 12 & 1 & 0 & -2.665297 & 2. 136507 & 1. 030099 \\
\hline 13 & 6 & 0 & 3. 984551 & -1.139392 & -1.124306 \\
\hline 14 & 1 & 0 & 0.513049 & 5. 422341 & 0.958721 \\
\hline 15 & 6 & 0 & 0.267645 & 3.547660 & 0.137432 \\
\hline 16 & 6 & 0 & -1.287836 & 0.826622 & 0.173537 \\
\hline 17 & 6 & 0 & -0.788574 & 3. 170889 & 0.926576 \\
\hline 18 & 6 & 0 & 0.695824 & 2. 803159 & -1.042095 \\
\hline 19 & 6 & 0 & 0.596586 & 1. 474831 & -1.223065 \\
\hline
\end{tabular}




$\begin{array}{rrrrrr}20 & 6 & 0 & -1.626055 & 2.015765 & 0.733356 \\ 21 & 1 & 0 & -1.111963 & 3.874426 & 1.692912 \\ 22 & 1 & 0 & 1.125842 & 3.379138 & -1.860031 \\ 23 & 1 & 0 & 0.937687 & 1.012029 & -2.144524 \\ 24 & 7 & 0 & 0.082541 & 0.608379 & -0.222268 \\ 25 & 1 & 0 & 1.502702 & 5.115540 & -0.377707 \\ 26 & 1 & 0 & 0.476541 & -2.643039 & 2.688736 \\ 27 & 6 & 0 & -2.263041 & -0.245709 & -0.133098 \\ 28 & 6 & 0 & -3.404620 & -0.442704 & 0.665928 \\ 29 & 6 & 0 & -2.079632 & -1.092132 & -1.241093 \\ 30 & 6 & 0 & -4.337094 & -1.430993 & 0.353848 \\ 31 & 1 & 0 & -3.546024 & 0.160774 & 1.556989 \\ 32 & 6 & 0 & -3.012215 & -2.082030 & -1.552443 \\ 33 & 1 & 0 & -1.203931 & -0.966312 & -1.870468 \\ 34 & 6 & 0 & -4.149542 & -2.255821 & -0.759745 \\ 35 & 1 & 0 & -5.205747 & -1.567461 & 0.992149 \\ 36 & 1 & 0 & -2.849577 & -2.717244 & -2.418827 \\ 37 & 1 & 0 & -4.874171 & -3.028352 & -0.999715\end{array}$

\section{S2-TS1 in the reaction of adenine with 4-biphenylylnitrenium ion at B3LYP/6-31+G(d,p) leve1}

Zero-point correction=

Thermal correction to Energy=

Thermal correction to Enthalpy=

Thermal correction to Gibbs Free Energy=

Sum of electronic and zero-point Energies=

Sum of electronic and thermal Energies=

Sum of electronic and thermal Enthalpies=

Sum of electronic and thermal Free Energies=

\begin{tabular}{cccccc}
$\begin{array}{c}\text { Center } \\
\text { Number }\end{array}$ & $\begin{array}{c}\text { Atomic } \\
\text { Number }\end{array}$ & $\begin{array}{c}\text { Atomic } \\
\text { Type }\end{array}$ & \multicolumn{2}{c}{ Coordinates } & (Angstroms) \\
- & 6 & 0 & -4.767841 & -1.168076 & 0.197166 \\
1 & 6 & 0 & -4.043876 & -0.865313 & 1.354248 \\
2 & 6 & 0 & -2.903756 & -0.068848 & 1.277732 \\
3 & 6 & 0 & -2.447794 & 0.420033 & 0.033704 \\
4 & 6 & 0 & -3.189594 & 0.101554 & -1.125397 \\
5 & 6 & 0 & -4.339132 & -0.677446 & -1.041809 \\
6 & 6 & 0 & -1.224516 & 1.231563 & -0.053275 \\
7 & 6 & 0 & -0.436761 & 1.240411 & -1.186116 \\
8 & 6 & 0 & 0.725396 & 2.063208 & -1.270013 \\
9 & 6 & 0 & 0.997348 & 3.056689 & -0.178798 \\
10 & 6 & 0 & 0.157784 & 2.974823 & 1.016719 \\
11 & 6 & 0 & -0.870621 & 2.102666 & 1.076392 \\
12 & 6 & 0 & 1.963375 & 3.878728 & -0.386265 \\
13 & 7 & 0 & 2.330514 & 0.931244 & -1.110625 \\
14 & 7 & 0 & 2.277955 & -0.121617 & -0.159488 \\
15 & 6 & 0 & 1.852663 & -1.398255 & -0.518986 \\
16 & 6 & 0 & 1.842712 & -2.337514 & 0.537811 \\
17 & 6 & 0 & 2.174005 & -2.094831 & 1.800778 \\
18 & 7 & & & &
\end{tabular}

0. 301604 (Hartree/Particle)

0.319280

0. 320224

0. 253414

$-984.842372$

$-984.824697$

$-984.823752$

$-984.890562$ 


\begin{tabular}{rrrrrr}
19 & 6 & 0 & 2.548003 & -0.825339 & 2.007802 \\
20 & 7 & 0 & 2.612677 & 0.164555 & 1.095639 \\
21 & 7 & 0 & 1.431442 & -3.507951 & -0.053578 \\
22 & 6 & 0 & 1.227537 & -3.229046 & -1.388497 \\
23 & 7 & 0 & 1.466126 & -1.976148 & -1.710081 \\
24 & 1 & 0 & 1.323414 & -4.403469 & 0.405185 \\
25 & 1 & 0 & 0.903166 & -3.993376 & -2.082031 \\
26 & 1 & 0 & -1.496778 & 2.077356 & 1.960659 \\
27 & 1 & 0 & 2.094620 & 4.523139 & 0.399442 \\
28 & 1 & 0 & 0.381713 & 3.640289 & 1.845497 \\
29 & 1 & 0 & 1.047486 & 2.399206 & -2.251858 \\
30 & 1 & 0 & -0.681113 & 0.605144 & -2.029797 \\
31 & 1 & 0 & 3.028774 & 1.640368 & -0.872181 \\
32 & 1 & 0 & 2.466963 & 0.576350 & -2.057827 \\
33 & 1 & 0 & 2.837509 & -0.561956 & 3.019879 \\
34 & 1 & 0 & -2.348732 & 0.139211 & 2.186447 \\
35 & 1 & 0 & -2.890894 & 0.503908 & -2.087665 \\
36 & 1 & 0 & -4.369902 & -1.246025 & 2.316873 \\
37 & 1 & 0 & -4.910113 & -0.892042 & -1.939528 \\
38 & 1 & 0 & -5.666121 & -1.774241 & 0.260644 \\
& 1 & & & & \\
\hline
\end{tabular}

S2-TS1 in the reaction of adenine with 4-biphenylylnitrenium ion at B3PW91/6-31+G(d,p) leve1

Zero-point correction=

Thermal correction to Energy=

Thermal correction to Enthalpy=

Thermal correction to Gibbs Free Energy=

Sum of electronic and zero-point Energies=

Sum of electronic and thermal Energies=

Sum of electronic and thermal Enthalpies=

Sum of electronic and thermal Free Energies=

\begin{tabular}{|c|c|c|c|c|c|}
\hline \multirow{2}{*}{$\begin{array}{l}\text { Center } \\
\text { Number }\end{array}$} & \multirow{2}{*}{$\begin{array}{l}\text { Atomic } \\
\text { Number }\end{array}$} & \multirow{2}{*}{$\begin{array}{l}\text { Atomic } \\
\text { Type }\end{array}$} & \multicolumn{3}{|c|}{ Coordinates (Angstroms) } \\
\hline & & & $X$ & $\mathrm{Y}$ & Z \\
\hline 1 & 6 & 0 & -4.754450 & -1.057000 & 0.188666 \\
\hline 2 & 6 & 0 & -4.016255 & -0.793843 & 1. 344050 \\
\hline 3 & 6 & 0 & -2.860453 & -0.024940 & 1. 273348 \\
\hline 4 & 6 & 0 & -2.402869 & 0.473293 & 0.035758 \\
\hline 5 & 6 & 0 & -3.158031 & 0.192966 & -1.122212 \\
\hline 6 & 6 & 0 & -4.324086 & -0.556551 & -1.043341 \\
\hline 7 & 6 & 0 & -1.169665 & 1. 258634 & -0.046589 \\
\hline 8 & 6 & 0 & -0.396764 & 1. 279271 & -1.193482 \\
\hline 9 & 6 & 0 & 0.770158 & 2. 075088 & -1.268970 \\
\hline 10 & 6 & 0 & 1. 073023 & 3. 038325 & -0.171654 \\
\hline 11 & 6 & 0 & 0.257487 & 2. 945201 & 1. 035976 \\
\hline 12 & 6 & 0 & -0.785261 & 2. 091535 & 1. 093636 \\
\hline 13 & 7 & 0 & 2. 038956 & 3. 858509 & -0.382728 \\
\hline 14 & 7 & 0 & 2. 391252 & 0.864676 & -1.080862 \\
\hline 15 & 6 & 0 & 2. 274801 & -0.182589 & -0.149084 \\
\hline 16 & 6 & 0 & 1. 797444 & -1.435867 & -0.525278 \\
\hline 17 & 6 & 0 & 1. 728601 & -2.378163 & 0.522850 \\
\hline 18 & 7 & 0 & 2. 046678 & -2.163245 & 1. 791787 \\
\hline
\end{tabular}

0. 302481 (Hartree/Particle)

0.320239

0. 321183

0. 253941

$-984.457528$

$-984.439770$

$-984.438825$

$-984.506068$ 


\begin{tabular}{rrrrrr}
19 & 6 & 0 & 2.471924 & -0.915324 & 2.011879 \\
20 & 7 & 0 & 2.597597 & 0.076012 & 1.113883 \\
21 & 7 & 0 & 1.278598 & -3.521872 & -0.083211 \\
22 & 6 & 0 & 1.111665 & -3.226508 & -1.414389 \\
23 & 7 & 0 & 1.409211 & -1.985367 & -1.723835 \\
24 & 1 & 0 & 1.125563 & -4.414479 & 0.366009 \\
25 & 1 & 0 & 0.766856 & -3.971930 & -2.119345 \\
26 & 1 & 0 & -1.400480 & 2.060255 & 1.986278 \\
27 & 1 & 0 & 2.183782 & 4.486134 & 0.413140 \\
28 & 1 & 0 & 0.505149 & 3.589945 & 1.874903 \\
29 & 1 & 0 & 1.134406 & 2.386515 & -2.244361 \\
30 & 1 & 0 & -0.664216 & 0.663796 & -2.045398 \\
31 & 1 & 0 & 3.093780 & 1.556916 & -0.816613 \\
32 & 1 & 0 & 2.528743 & 0.523088 & -2.030671 \\
33 & 1 & 0 & 2.755196 & -0.672666 & 3.031795 \\
34 & 1 & 0 & -2.291761 & 0.151738 & 2.181029 \\
35 & 1 & 0 & -2.856910 & 0.606585 & -2.079618 \\
36 & 1 & 0 & -4.344767 & -1.184873 & 2.302077 \\
37 & 1 & 0 & -4.907676 & -0.741076 & -1.940031 \\
38 & 1 & 0 & -5.667328 & -1.642102 & 0.248578 \\
& 1 & & & & \\
\hline
\end{tabular}

S2-INT2 in the reaction of adenine with 4-biphenylylnitrenium ion at B3PW91/6-31+G(d,p) leve1

\section{Zero-point correction=}

Thermal correction to Energy=

Thermal correction to Enthalpy=

Thermal correction to Gibbs Free Energy=

Sum of electronic and zero-point Energies=

Sum of electronic and thermal Energies=

Sum of electronic and thermal Enthalpies=

Sum of electronic and thermal Free Energies=

$\begin{array}{ccc}\text { Center } & \text { Atomic } & \text { Atomic } \\ \text { Number } & \text { Number } & \text { Type }\end{array}$

0. 304410 (Hartree/Particle)

0. 322081

0. 323025

0. 257184

$-984.508958$

$-984.491288$

$-984.490344$

$-984.556185$

\begin{tabular}{|c|c|c|c|c|c|}
\hline 1 & 6 & 0 & -0.260004 & 1. 019682 & 0.131953 \\
\hline 2 & 6 & 0 & -0.175493 & 1. 066643 & 1. 523755 \\
\hline 3 & 6 & 0 & 1. 038840 & 0.819208 & 2. 159402 \\
\hline 4 & 6 & 0 & 2. 184794 & 0.514393 & 1. 409371 \\
\hline 5 & 6 & 0 & 2. 090197 & 0.473680 & 0.010517 \\
\hline 6 & 6 & 0 & 0.875212 & 0.723327 & -0.622803 \\
\hline 7 & 6 & 0 & 3. 472285 & 0.231321 & 2. 083546 \\
\hline 8 & 6 & 0 & 4. 303615 & -0.767742 & 1. 690235 \\
\hline 9 & 6 & 0 & 5. 664911 & -0.969186 & 2. 285516 \\
\hline 10 & 6 & 0 & 5. 863472 & -0.280348 & 3. 612551 \\
\hline 11 & 6 & 0 & 4. 995880 & 0.778825 & 3. 973931 \\
\hline 12 & 6 & 0 & 3. 873280 & 1. 018165 & 3. 229095 \\
\hline 13 & 7 & 0 & 6.919257 & -0.613597 & 4. 323004 \\
\hline 14 & 7 & 0 & 6. 150188 & -2.331810 & 2. 323881 \\
\hline 15 & 6 & 0 & 5. 389615 & -3.255841 & 3. 019408 \\
\hline 16 & 6 & 0 & 5.568030 & -4.636356 & 2. 883440 \\
\hline 17 & 6 & 0 & 4. 734074 & -5.425343 & 3. 690752 \\
\hline 18 & 7 & 0 & 3. 817557 & -4.986149 & 4. 555238 \\
\hline
\end{tabular}




$\begin{array}{rrrrrr}19 & 6 & 0 & 3.749148 & -3.660533 & 4.589497 \\ 20 & 7 & 0 & 4.481147 & -2.782941 & 3.871901 \\ 21 & 7 & 0 & 5.079575 & -6.712551 & 3.375327 \\ 22 & 6 & 0 & 6.069332 & -6.637871 & 2.423724 \\ 23 & 7 & 0 & 6.393283 & -5.408624 & 2.099174 \\ 24 & 1 & 0 & 4.675299 & -7.550840 & 3.769028 \\ 25 & 1 & 0 & 6.517372 & -7.528098 & 2.002236 \\ 26 & 1 & 0 & 3.224555 & 1.837586 & 3.528889 \\ 27 & 1 & 0 & 7.143658 & -0.150250 & 5.192978 \\ 28 & 1 & 0 & 5.205184 & 1.366973 & 4.862525 \\ 29 & 1 & 0 & 6.349853 & -0.412381 & 1.615981 \\ 30 & 1 & 0 & 4.046215 & -1.419774 & 0.859788 \\ 31 & 1 & 0 & 7.460889 & -1.426875 & 4.050045 \\ 32 & 1 & 0 & 6.640239 & -2.686497 & 1.512463 \\ 33 & 1 & 0 & 3.021185 & -3.225792 & 5.268641 \\ 34 & 1 & 0 & 1.082515 & 0.832769 & 3.245846 \\ 35 & 1 & 0 & 2.976766 & 0.276745 & -0.586818 \\ 36 & 1 & 0 & -1.057448 & 1.290219 & 2.116413 \\ 37 & 1 & 0 & 0.818267 & 0.699306 & -1.706937 \\ 38 & 1 & 0 & -1.206001 & 1.218137 & -0.362721 \\ & & & & & \\ ------------------------------------------------------------\end{array}$

